\title{
On the Construction of Monopoles for the Classical Groups ${ }^{\star}$
}

\author{
Jacques Hurtubise ${ }^{1}$ and Michael K. Murray ${ }^{2}$ \\ ${ }^{1}$ School of Mathematics, Institute for Advanced Study, Princeton, N.J. 08540, USA and Department \\ of Mathematics, McGill University, Burnside Hall, 805 Sherbrooke St. W., Montreal, Quebec, Canada \\ H3A 2 K6 \\ ${ }^{2}$ Department of Mathematics. R.S. Phys. S., The Australian National University, GPO Box 4, \\ Canberra, ACT 2601, Australia
}

\begin{abstract}
For $G$ a classical group, an equivalence is exhibited between:
A) $G$ monopoles over $\mathbb{R}^{3}$, with maximal symmetry breaking at infinity,

B) families of $(\operatorname{rank}(G))$ algebraic curves in $T \mathbb{P}_{1}$, along with divisors on those curves, satisfying certain constraints,

C) solutions of Nahm's equations over $(\operatorname{rank}(G))$ intervals, satisfying the appropriate boundary conditions.

A) and B) are linked by twistor techniques, B) and C) via the Krichever method for solving non-linear differential equations, and A) and C) via the ADHMN construction, providing a unified picture of techniques for solution. Amongst other things, an asymptotic formula for the Higgs field of the monopole is computed.
\end{abstract}

\section{Contents}

Introduction . . . . . . . . . . . . . . . . . . . . . . . . . . . . . 36

1. From Monopoles to Spectral Data . . . . . . . . . . . . . 42

a) Bundles and Flag Structures . . . . . . . . . . . . . . . . . . 42

b) A Meromorphic Reduction to a Torus . . . . . . . . . . . . 45

c) A Vanishing Theorem . . . . . . . . . . . . . . . . . . . . . . . 48

d) The Asymptotic Higgs Field . . . . . . . . . . . . . . . . . . . 51

e) Reality Conditions . . . . . . . . . . . . . . . . . . . . . . 53

2. From Spectral Data to Nahm's Equations . . . . . . . . . . . 56

a) Introduction and Notation . . . . . . . . . . . . . . . . . 56

b) Solution Over $\left(\mu_{p+1}, \mu_{p}\right)$. . . . . . . . . . . . . . . . . . . . . 57

c) Behaviour of $X$ at $\mu_{p}$. . . . . . . . . . . . . . . . . . . . . . . . 58

* Research supported in part by NSERC grant A8361 and FCAR grant EQ3518 
d) The Endomorphism $\underline{A}(z, \zeta)$ at $\mu_{p}$.

e) The Matrices $A(z, \zeta)$ at $z=0$. . . . . . . . . . . . . 68

f) Real Structure.

3. From Nahm's Equations to Spectral Data . . . . . . . . . . . 71

4. Reconstructing the Monopole . . . . . . . . . . . . . . . 77

a) Introduction . . . . . . . . . . . . . . . . . . . 77

b) The ADHMN Construction . . . . . . . . . . . . . . . . 77

c) Link to the Twistor Approach . . . . . . . . . . . . . . 80

d) The Equivalence of the Connections and of the Higgs Fields . . . 84

5. Modifications for the Cases $S O(k), S p(k)$. . . . . . . . . . . . 85

a) From Monopoles to Spectral Data . . . . . . . . . . . . 85

b) From Spectral Data to Nahm's Equations . . . . . . . . . . 87

6. Summary and Conclusion. . . . . . . . . . . . . . . . . 87

References . . . . . . . . . . . . . . . . 88

\section{Introduction}

In recent years, monopoles have been studied quite extensively, from different points of view. One method is direct, involving analysis [JT, T1, T2]; another is complex-analytic, and employs twistor methods [W, Hi1]; yet another, due to $\mathrm{Nahm}[\mathrm{N}]$ is an infinite dimensional version of the algebraic ADHM construction of instantons, and involves the solution of some non-linear ordinary differential equations, Nahm's equations.

From all of this, a fairly complete picture has emerged of the $S U(2)$-case. In particular, a beautiful paper of Hitchin [Hi2] gives the equivalence between

- an $S U(2)$-monopole

--an algebraic curve in $T \mathbb{P}_{1}(\mathbb{C})$ satisfying certain constraints

- a solution to Nahm's equations satisfying the appropriate boundary condition.

Using this equivalence, Donaldson [D] was able to give a description of the moduli space. Recently, the dynamics of monopoles have been studied in terms of geodesic motion on this space [AHi].

Our aim is to extend these results of Hitchin to the other classical groups, in the case of maximal symmetry breaking at infinity. As in [Hi2], we will prove an equivalence between three types of objects. These are defined as follows:

I) The Case of $S U(N)$. Our objects are:

$A(S U)$ : $S U(N)$ Monopoles: Let $H$ be a rank $N$ complex vector bundle over $\mathbb{R}^{3}$. Let $\nabla$ be an $S U(N)$ connection on $H$, and let $\Phi$ (the "Higgs field") be a section of the associated $s u(N)$ adjoint bundle. $(H, \nabla, \Phi)$ is an $S U(N)$ monopole if

A1) $(\nabla, \Phi)$ satisfies the Bogomoln'yi equation, $* F=\nabla \phi$, where $F$ is the curvature of $\nabla$ and $*$ is the Hodge duality operator.

A2) One has uniform asymptotic expansions, up to gauge transformation,

$$
\begin{aligned}
& \Phi=i \operatorname{diag}\left(\mu_{j}-\left(k_{j} / 2 r\right)\right)+O\left(1 / r^{2}\right), \\
& |\nabla \Phi|=O\left(1 / r^{2}\right), \quad \text { and }
\end{aligned}
$$




$$
\frac{\partial|\Phi|}{\partial \Omega} \stackrel{\text { def }}{=}\left(\left(\frac{\partial|\Phi|}{\partial \theta}\right)^{2}+\sin ^{2} \theta\left(\frac{\partial|\Phi|}{\partial \rho}\right)^{2}\right)^{1 / 2}=O\left(1 / r^{2}\right),
$$

where $(r, \theta, \rho)$ are spherical coordinates in $\mathbb{R}^{3}$.

The $\mu_{j}$ and $k_{j}$ are fixed, independent of direction and satisfy $\Sigma \mu_{j}=\Sigma k_{j}=0$. The condition of maximal symmetry breaking is that the $\mu_{j}$ are distinct; we order them by

$$
\mu_{1}>\mu_{2}>\cdots>\mu_{N}
$$

The $k_{j}$ 's are integers; we define the $p^{\text {th }}$ magnetic charge $m_{p}, p=1, \ldots, N-1$, by

$$
m_{p}=k_{1}+\cdots+k_{p}
$$

The second type of objects we are going to study is

$B(S U): N a h m$ Data. In the $S U(N)$ case, one has analytic hermitian vector bundles $X_{p}$ of rank $m_{p}$ on the intervals $\left[\mu_{p+1}, \mu_{p}\right]$, with, on the interior of each interval, an analytic hermitian connection $\nabla_{t}$ and three analytic skew-hermitian endomorphisms $T_{i}(t)$ satisfying:

B1) Nahm's Equations:

$$
\nabla_{t} T_{i}=\frac{1}{2} \sum \varepsilon_{l j k}\left[T_{j}, T_{k}\right]
$$

B2) Boundary Conditions. We adopt the convention $m_{0}=m_{N}=0$. At a boundary point $\mu_{p}$, we distinguish three cases:

i) $m_{p}>m_{p-1}$ :

In this case, there should be at $\mu_{p}$ an injection $X_{p-1} \rightarrow X_{p}$, compatible with the hermitian structure such that

--there exist well defined limits from above:

$$
T_{i}^{+}=\lim _{t \rightarrow \mu_{p}+} T_{i}(t)
$$

- for $t<\mu_{p}$, setting $z=t-\mu_{p}$, one has in a covariant constant basis, the expansion:

$$
\begin{aligned}
& k_{p} \quad m_{p-1} \\
& T_{i}(z)=\left(\frac{r_{i} / z+O(1)}{O\left(z^{(k-1) / 2}\right)}-\frac{O\left(z^{(k-1) / 2}\right.}{T_{i}^{+}+O(z)}\right) \begin{array}{l}
k_{p} \\
m_{p-1}
\end{array} .
\end{aligned}
$$

The diagonal blocks are meromorphic; the off-diagonal blocks are $z^{((k-1) / 2)} \times$ analytic. The residues $r_{l}, i=1,2,3$ define an irreducible $k_{p}$-dimensional representation of $\operatorname{su}(2)$.

ii) $m_{p}<m_{p-1}$ :

One imposes the same boundary conditions, but with the roles of $\left(\mu_{p+1}, \mu_{p}\right)$, $\left(\mu_{p}, \mu_{p-1}\right)$ reversed.

iii) $m_{p}=m_{p-1}$ :

We then have an identification at $\mu_{p}$ of $X_{p}$ with $X_{p-1}$, such that if one sets

$$
A(t, \zeta)=\left(T_{1}(t)+i T_{2}(t)\right)+2 i T_{3}(t) \zeta+\left(T_{1}(t)-i T_{2}(t)\right) \zeta^{2}
$$


one asks that the one-sided limits $A^{+}(\zeta), A^{-}(\zeta)$ of $A(t, \zeta)$ exist at $\mu_{p}$, and that $A^{+}(\zeta)-A^{-}(\zeta)$ be at most of rank one, for all $\zeta$. This is equivalent to asking that there be vectors $u_{0}, u_{1}, \in \mathbb{C}^{m}$ with

$$
A^{+}(\zeta)-A^{-}(\zeta)=\left(u_{0}+u_{1} \zeta\right)\left(\bar{u}_{1}-\bar{u}_{0} \zeta\right)^{T} .
$$

For both $S U(N)$ monopoles and solutions to Nahm's equations we can define spectral curves $S_{i} \subset T \mathbb{P}_{1}(\mathbb{C}), i=1, \ldots, N-1$. For monopoles, this is outlined in Sect. 1; for the case of Nahm's equations, let $\zeta$ be an affine coordinate on $\mathbb{P}_{1}$, and let $\eta$ be the associated fiber coordinate in $T \mathbb{P}_{1}$; the $i^{\text {th }}$ spectral curve is defined by

$$
\operatorname{det}(\eta \vartheta-A(t, \zeta))=0
$$

for $t \in\left(\mu_{i+1}, \mu_{i}\right)$. Nahm's equations are isospectral, so this is independent of the $t$ chosen. Let $\mathcal{O}(k)$ denote the lift to $T \mathbb{P}_{1}$ of the line bundle $\mathcal{O}(k)$ on $\mathbb{P}_{1}$; in both cases the curves $S_{p}$ belong to the linear system $\left|\circlearrowleft\left(2 m_{p}\right)\right|$, and are compact. We will say that the monopole or the Nahm's data is generic if

$$
S_{p} \cap S_{p-1} \text { consists of } 2 m_{p} m_{p-1} \text { distinct points, for } p=2, \ldots, N-1 \text {, }
$$

i.e. $S_{p}$ and $S_{p-1}$ intersect transversally

It is a non-trivial fact that generic monopoles and Nahm data exist. Let $L^{\mu}(k)$ denote the line bundle over $T \mathbb{P}_{1}$ with transition function $\exp (\mu \eta / \zeta) \zeta^{k}$ from $U_{1}=\{\zeta \neq 0\}$ to $U_{0}=\{\zeta \neq \infty\}$. Let $\tau: T \mathbb{P}_{1} \rightarrow T \mathbb{P}_{1}$ denote the real structure $\tau(\eta, \zeta)=$ $\left(-\bar{\eta} / \bar{\zeta}^{2},-1 / \bar{\zeta}\right)$. We will show that, from a generic monopole, or generic Nahm data, one can extract:

$C(S U)$ Spectral Data. This consists of the compact, real ( $\tau$-invariant) curves $S_{p} \in\left|\mathcal{O}\left(2 m_{p}\right)\right|, p=1, \ldots, N-1$, in generic position, along with a splitting

$$
S_{p} \cap S_{p-1}=S_{p, p-1} \cup S_{p-1, p} \quad p=2, \ldots, N-1
$$

into disjoint subsets of points of equal cardinality, such that

C1) Over $S_{p}$

$$
\mathcal{O} \cong L^{\mu_{p+1}-\mu_{p}}\left(m_{p-1}+m_{p+1}\right)\left[-S_{p \cdot p+1}-S_{p, p-1}\right] .
$$

C2) One has the vanishing theorem

$$
H^{0}\left(S_{p}, L^{\left(\mu_{p}-z\right)}\left(m_{p}+m_{p-1}-2\right)\left[-S_{p-1 . p}\right]\right)=0
$$

for a) $\mu_{p+1}<z<\mu_{p}$

b) $z=\mu_{p}$ if $m_{p-1} \geqq m_{p}$

and c) $z=\mu_{p+1}$ if $m_{p} \leqq m_{p+1}$.

C3) The reality constraint

$$
\tau\left(S_{p, p+1}\right)=S_{p+1, p} .
$$

C4) The positivity constraint.

Let $\psi_{p}$ be the section realizing the isomorphism in $\mathrm{C} 1$; set $\psi_{p}^{*}=\tau^{*}\left(\psi_{p}\right)$. Then $\psi_{p} \psi_{p}^{*}$ cuts out in $S_{p}$ the divisor of $S_{p+1} \cap S_{p-1}$; the union of these curves can be 
given real equation

$$
g_{p-1} g_{p+1}=\eta^{m_{p-1}+m_{p+1}}+(\text { lower order in } \eta) .
$$

One then has that $\left(\psi_{p} \psi_{p}^{*}\right) /\left(g_{p-1} g_{p+1}\right)$ is a real constant $e_{p}$; one asks that

$$
-(-1)^{m_{p}+m_{p-1}} e_{p}>0 \text {. }
$$

II) The Cases of $S O(k), S p(k)$. For the other classical groups, the definitions given above must be modified somewhat.

$A(S O), A(S p)$ : G-Monopoles, $G=S O(k), S p(k)$. One adds an orthogonal or symplectic structure to the bundle $H$ over $R^{3} ; \nabla$ is then compatible with this structure, and $\Phi$ is a section of the associated $s o-, s p$-adjoint bundle.

We will treat the case of $S O$-, $S p$-monopoles as $S U$-monopoles endowed with extra structure. One has the following table: (note $[\mathrm{M}]$ that a $G$-monopole has rank $(G)$ magnetic charges):

\begin{tabular}{|c|c|c|c|c|}
\hline $\begin{array}{l}\text { A } G \text {-monopole } \\
\text { for } G=\end{array}$ & $\begin{array}{l}\text { with } \\
G \text {-charges }\end{array}$ & $\begin{array}{l}\text { embedded } \\
\text { in } S U(N)\end{array}$ & $\begin{array}{l}\text { As an } S U(N) \text { - } \\
\text { monopole, its } \\
\text { Higgs field is } \\
\text { asymptotic to } \\
\text { diag }\left(\mu_{l}\right) \text { with: }\end{array}$ & $\begin{array}{l}\text { and it has } \\
S U(N) \text { charges } \\
m_{l}, \text { with }\end{array}$ \\
\hline$S p(k)$ & $r_{1}, \ldots, r_{k}$ & $N=2 k$ & $\begin{aligned} \mu_{l} & =-\mu_{2 h+1-l} \\
l & =1 \ldots, k\end{aligned}$ & $\begin{aligned} m_{l} & =m_{2 k-1}=r_{1} \\
l & =1, \ldots, k\end{aligned}$ \\
\hline$S O(2 k)$ & $\begin{array}{l}r_{1}, \ldots, r_{h-2}, \\
r_{+}, r_{\ldots}\end{array}$ & $N=2 k$ & $\begin{aligned} \mu_{l} & =-\mu_{2 h+1 \cdots t} \\
i & =1, \ldots k\end{aligned}$ & $\begin{array}{l}m_{t}=m_{2 k-t}=r_{t} \\
\quad i=1, \ldots, k-2 \\
m_{k-1}=m_{k+1}=r_{+}+r_{-} \\
m_{k}=2 r_{+} .\end{array}$ \\
\hline$S O(2 k+1)$ & $r_{1}, \ldots, r_{k}$ & $N=2 k+1$ & $\begin{aligned} \mu_{1} & =-\mu_{2 h+2-i} \\
i & =1, \ldots, k+1\end{aligned}$ & $\begin{aligned} m_{l} & =m_{2 k+1-1}=r_{\imath} \\
i & =1, \ldots, k-1 \\
m_{h} & =m_{h+1}=2 r_{h}\end{aligned}$ \\
\hline
\end{tabular}

With this table in mind, one asks that the monopole conditions A-1 and A-2 again be satisfied.

$B(S O), B(S p)$ : Nahm Data. In these cases, referring to (0.4), the Nahm data is the same as in the $S U(N)$-case, with the added condition:

(B-3) There are matrices $C_{j}$ such that, for $z \in\left(\mu_{j+1}, \mu_{j}\right), i=1,2,3$,

$$
T_{i}(-z)^{T}=C_{j} T_{i}(z) C_{j}^{-1} .
$$

$C_{j}$ and $C_{j-1}$ are compatible in the obvious way at $\mu_{j}$. Also,

$$
C_{N-j+1}=-C_{j}^{T} \text { for } S O, C_{j}^{T} \text { for } S p .
$$

$C(S O), C(S p):$ Spectral Data:

$C(S p)$ : For $S p(k)$ monopoles, one can define [M] spectral curves $R_{p} \in\left|\mathcal{O}\left(2 r_{p}\right)\right|$, $p=1, \ldots, k$. The genericity condition is that $R_{p} \cap R_{p+1}$ consist of $2 r_{p} r_{p+1}$ distinct points, i.e. that the intersection of $R_{p}$ and $R_{p+1}$ be transversal. Under (0.4), the 
$S U$-spectral curves are:

$$
S_{p}=S_{2 k-p}=R_{p}, \quad i=1, \ldots, k
$$

The conditions $C(S p)$ are then exactly those of $C(S U)$.

$C(S O(2 k)):$ Here, $[\mathrm{M}]$ yields spectral curves $R_{1}, \ldots, R_{k-2}, R_{+}, R_{-}, R_{p} \in\left|\mathcal{O}\left(2 r_{p}\right)\right|$, $R_{ \pm} \in\left|\mathcal{O}\left(2 r_{ \pm}\right)\right|$. The genericity conditions are that $R_{p}$ and $R_{p+1}, p=1, \ldots, k-3, R_{+}$ and $R_{-}, R_{k-2}$ and $\left(R_{+} \cup R_{-}\right)$intersect transversally. Under (0.4), the associated $S U$-spectral curves are:

$$
\begin{aligned}
S_{p} & =S_{2 k-p}=R_{p}, \quad p=1, \ldots, k-2, \\
S_{k-1} & =S_{k+1}=R_{+} \cup R_{-}, \\
S_{k} & =2 R_{+} \text {(i.e., with multiplicity two). }
\end{aligned}
$$

Also

$$
\begin{aligned}
& S_{p, p+1}=S_{2 k-p, 2 k-p-1}, \\
& S_{p+1, p}=S_{2 k-p-1,2 k-p} .
\end{aligned}
$$

The conditions on $S_{1}, \ldots, S_{k-2}, S_{k+2}, \ldots, S_{2 k-1}$ are then exactly the same as for $S U$. In addition, one has:

$C-1_{ \pm}$) Over $R_{+}$,

$$
0 \approx L^{\mu_{k-1}-\mu_{k}}\left(m_{k-2}\right)\left[-S_{k-2, k-1}\right]
$$

and over $R_{-}$,

$$
\mathcal{O} \approx L^{\mu_{k-1}+\mu_{k}}\left(m_{k-2}\right)\left[-S_{k-1, k-2}\right] .
$$

$\left.C-2_{k-1}\right)$ For $S_{k-1}$, the same vanishing theorem as for $S U$ $C-2_{k}$ ) The isomorphisms $C-1_{ \pm}$yield an identification

$$
L^{-\mu_{k}}\left(m_{k-1}\right) \approx L^{\mu_{k}}\left(m_{k-1}\right)
$$

over $R_{+} \cap R_{-}$. Define the bundle $Q_{k}$ over $R_{+}$by the exact sequence.

$$
\left.0 \rightarrow Q_{k} \rightarrow L^{\mu_{k}}\left(m_{k-1}\right) \oplus L^{-\mu_{k}}\left(m_{k-1}\right) \rightarrow L^{\mu_{k}}\left(m_{k-1}\right)\right|_{R_{+} \cap R_{-}} \rightarrow 0 .
$$

The vanishing theorem is then:

$$
\begin{aligned}
H^{0}\left(R_{+}, Q_{k} \otimes L^{-z}(-2)\right)=0 \quad \text { for } & \text { a) }-\mu_{k} \leqq z \leqq \mu_{k} \\
& \text { b) } z=\mu_{k}, \text { if } \quad r_{+} \leqq r_{\ldots} .
\end{aligned}
$$

$\left.C-2_{k+1}\right)$ The vanishing theorem is:

$$
\begin{aligned}
& H^{0}\left(S_{k+1}, L^{\left(\mu_{k+2}-z\right)}\left(m_{k+1}+m_{k+2}-2\right)\left[-S_{k+1 . k+2}\right]\right)=0 \text { for } \\
& \text { a) } \mu_{k+2 \leqq z \leqq \mu_{k+1}} \\
& \text { b) } z=\mu_{k+1} \text { if } r_{+} \geqq r_{-} \\
& \text {c) } z=\mu_{k+2} \text { if } m_{k+1} \leqq m_{k+2}
\end{aligned}
$$

$C-4_{ \pm}$) The extra positivity constraints:

Let $\psi_{ \pm}$realize the isomorphisms in $C-1_{ \pm}$; then $\psi_{ \pm} \psi_{ \pm}^{*}=e_{ \pm} g_{k-2}$, with $g_{k-2}=\eta^{m_{k-2}}+$ 
(lower order in $\eta), e_{ \pm}$a real constant. One asks that:

$$
-(-1)^{\left(r_{k-2}+r_{ \pm}\right)} e_{ \pm}>0 .
$$

$C(S O(2 k+1)):$ In this case, one has spectral curves $R_{p} \in\left|O\left(2 r_{p}\right)\right|, p=1, \ldots, k$, with the genericity condition that $R_{p}$ and $R_{p+1}, p=1, \ldots, k-1$ intersect transversally. One has:

$$
\begin{aligned}
& S_{p}=S_{2 k+1-p}=R_{p}, \quad p=1, \ldots, k-1, \\
& S_{k}=S_{k+1}=2 R_{k} .
\end{aligned}
$$

Also,

$$
\begin{aligned}
& S_{p, p+1}=S_{2 k+1-p, 2 k-p}, \\
& S_{p+1, p}=S_{2 k-p, 2 k+1-p} .
\end{aligned}
$$

The conditions on $S_{1}, \ldots, S_{k-1}, S_{k+2}, \ldots, S_{2 k}$ are as in the $S U$-case. In addition, one has:

$C-1_{k}$ ) Over $R_{k}$

$$
\mathcal{O} \approx L^{\mu_{k}}\left(m_{k-1}\right)\left[-S_{k-1, k}\right] .
$$

$\left.\left.C-2_{k}\right), C-2_{k+1}\right)$ The same vanishing theorems over $S_{k}, S_{k+1}$ as in the $S U$-case.

$C-4_{k}$ ) The extra positivity constraint:

Let $\psi_{k}$ realize the isomorphism $C-1_{k}$; then $\psi_{k} \psi_{k}^{*}=e_{k} g_{k-1}, g_{k-1}=\eta^{m_{k-1}}+($ lower order in $\eta), e_{k}$ a real constant. Then

$$
\left.-(-1)^{\left(m_{k}-1+r_{k}\right.}\right) e_{k}>0 .
$$

It is thus our intention to prove, for the cases $G=S U(N), S O(k), S p(k)$ with maximal symmetry breaking:

Theorem 1. There is a natural equivalence between
A) Generic monopoles,
B) Generic Nahm data,
C) Spectral data.

The condition of genericity, using analyticity results of $[\mathrm{JT}]$, is a (real) Zariski-open one. Let $M_{m}, m=\left(m_{1}, \ldots, m_{\operatorname{rank}(G)}\right)$ be the union of the connected components of the charge $m G$ monopole moduli space which contain generic monopoles; elements of $M_{m}$ are limits of generic monopoles. One can then show

Theorem 2. There is a natural equivalence between
A) Monopoles in $M_{m}$
B) Nahm data.

This result is used in [Hu3] to describe $M_{m}$ in terms of rational maps of $\mathbb{P P}_{1}$ into flag manifolds; amongst other things, $M_{m}$ is connected. It is conjectured that the moduli space of charge $m$ monopoles is connected, and so is equal to $M_{m}$; this is indeed the case for $S U(2)$ and $S U(3)$ [T2]. For an arbitrary monopole, one still obtains curves, and a generalization of the vanishing theorem. This in turn enables 
us to define a solution to Nahm's equations; the problem lies in showing that it satisfies the boundary conditions.

The paper is organized as follows. In Sects. 1 to 4 , we concentrate on the case of $S U(N)$.

Section 1 is connected with the passage $A \Rightarrow C$. We recall from [M] how, from a monopole, we can obtain a holomorphic bundle $E$ over $T \mathbb{P}_{1}$, along with two flags of subbundles $E_{i}^{+}, E_{i}^{-}$. The spectral curve $S_{p}$ is (set-theoretically) the support of the sheaf $E /\left(E_{p}^{+}+E_{N-p}^{-}\right)$.

We then prove the vanishing theorem and show how in the generic case, the conditions on the spectral data are satisfied. We also show how one can construct $E$ from the spectral data, and derive an asymptotic formula for the Higgs field of the corresponding solution to the Bogomoln'yi equations in $\mathbb{R}^{3}$.

In Sect. 2, we study the correspondence $C \Rightarrow B$; from this we show how any monopole gives a solution to Nahm's equations, and prove that monopoles in $M_{m}$ give solutions satisfying the boundary conditions.

Section 3 gives the inverse of the construction of Sect. 2; from a generic solution to $B$, we obtain the spectral data $C$.

Section 4 is concerned with the ADHMN construction of a monopole from a solution to Nahm's equations. This construction is described; we also show that, in the generic case, under the equivalence given in Sects. 2 and 3, it gives the same monopole as the twistor construction. This fact is then exploited: regularity is immediate from the ADHMN point of view, whereas using the twistor construction, one easily obtains from the asymptotic formulae of Sect. 1 that $(\nabla, \Phi)$ satisfies the boundary conditions.

In Sect. 5 we explain very briefly how these constructions must be modified for the cases of $S O(k), S p(k)$.

Section 6 provides a summary and conclusion, showing that the circle of ideas does indeed close.

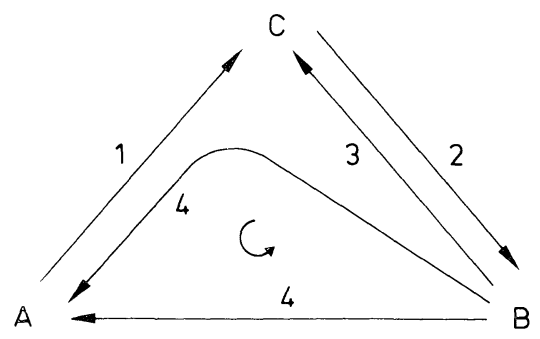

\section{From Monopoles to Spectral Data}

1a) Bundles and Flag Structures. In $[\mathrm{M}]$, it was shown that, from a solution $(H, \nabla, \Phi)$ to the $S U(N)$ Bogomolny equations over $\mathbb{R}^{3}$, one can obtain a rank $N(\operatorname{Sl}(N, \mathbb{C}))$ holomorphic vector bundle $E$ over $T \mathbb{P}_{1}(\mathbb{C})$. Recall [Hi1] that the space of oriented lines in $\mathbb{R}^{3}$ has a natural complex structure, and is holomorphically equivalent to $T \mathbb{P}_{1}(\mathbb{C})$. This correspondence can be given in coordinates as follows. 
Let $\zeta$ be an affine coordinate on $\mathbb{P}_{1}(\mathbb{C})$, and let $\eta$ be the corresponding fiber coordinate in $T \mathbb{P}_{1}(\mathbb{C})(\eta \rightarrow \eta d / d \zeta)$. Note that $T \mathbb{P}_{1}(\mathbb{C})$ is covered by two coordinate patches $U_{0}(\zeta \neq \infty)$ and $U_{1}(\zeta \neq 0)$ with coordinates $(\eta, \zeta)$ and $\left(\eta^{\prime}, \zeta^{\prime}\right)=\left(\eta / \zeta^{2}, 1 / \zeta\right)$ respectively. The correspondence between $x=\left(x_{1}, x_{2}, x_{3}\right) \in \mathbb{R}^{3}$ and $(\eta, \zeta)$ is then:

$$
\eta=\left(x_{1}+i x_{2}\right)-2 x_{3} \zeta+\left(-x_{1}+i x_{2}\right) \zeta^{2} .
$$

This can be viewed in two ways: fixing $\eta, \zeta$, it defines a line $l(\eta, \zeta)$ in $\mathbb{R}^{3}$; fixing $x$, it defines the image $C_{x}$ of a section $\mathbb{P}_{1} \rightarrow T \mathbb{P}_{1}$. Also, $T \mathbb{P}_{1}(\mathbb{C})$ has a real structure $\tau: T \mathbb{P}_{1} \rightarrow T \mathbb{P}_{1}$, given invariantly by reversal of orientation along a line, and in coordinates by $\tau(\eta, \zeta)=\left(-\bar{\eta} / \bar{\zeta}^{2},-1 / \bar{\zeta}\right)$.

If $l$ is a line in $\mathbb{R}^{3}$, define $E_{l}=$ space of solutions $s$ along $l$ to $\left(\nabla_{u}-i \Phi\right) s=0$, where $u$ is the positive unit vector field on $l$. As $(H, \nabla, \Phi)$ satisfies the Bogomoln'yi equations, $E$ has an integrable holomorphic structure [Hi1].

The fact that $\nabla$ is an $S U(N)$ connection, and that $\Phi$ is skew adjoint implies that if $\left(\nabla_{u}-i \Phi\right)_{s}=0$ along a line $l$, and if $\left(\nabla_{u}+i \Phi\right) t=0$, then $\partial / \partial u\langle s, t\rangle=0$. Thus the dual of $E_{l}$, via the isomorphism given by the metric on $H$, is the space of solutions to $\left(\nabla_{u}+i \Phi\right) t=0$. This however, is the same as the solutions to $\left(\nabla_{-u}-i \Phi\right) t=0$, i.e. $E_{\tau(l)}$. In short, there is an antilinear map $\sigma: E \rightarrow E^{*}$, lifting the $\operatorname{map} \tau: T \mathbb{P}_{1} \rightarrow T \mathbb{P}_{1}$.

If $(H, \nabla, \Phi)$ satisfies the boundary conditions $A-2$, then it is shown in [M] that $E$ possesses additional structure. Before recalling this, we again define some basic line bundles over $T \mathbb{P}_{1}$ : first, one has the pull-back from $\mathbb{P}_{1}$ of the standard line bundles $O(k), k \in \mathbb{Z}$. These have $\zeta^{k}$ as a standard transition function from $U_{1}$ to $U_{0}$, i.e. a section of $\mathcal{O}(k)$ is described by functions $f_{i}$ on $U_{i}$ with $f_{0}=\zeta^{k} f_{1}$ on the overlap. Next, define line bundles $L^{\mu}, \mu \in \mathbb{R}$ by the transition function $e^{\mu n / \zeta}$ from $U_{1}$ to $U_{0}$. If $F$ is any bundle, define $F(k)$ to be $F \otimes \mathcal{C}(k)$.

\section{Lemma 1.2.}

a) In the standard trivialisations over $U_{0}, H^{0}\left(T \mathbb{P}_{1}, O(k)\right)=$ polynomials in $\eta, \zeta$ of degree $\leqq k$, where degree $(\eta)=2$, degree $(\zeta)=1$. Therefore, $h^{0}\left(T \mathbb{P}_{1}, \mathcal{O}(2 j)\right)=$ $(j+1)^{2}$, and $h^{\mathrm{O}}\left(T \mathbb{P}_{1}, \mathcal{O}(2 j+1)\right)=(j+1)(j+2)$.

b) $H^{0}\left(T \mathbb{P}_{1}, L^{\mu}(k)\right)=0$, for all $\mu \neq 0$, for all $k$.

c) $H^{1}\left(T \mathbb{P}_{1}, \mathcal{O}(k)\right)=\mathcal{O}\left(U_{0} \cap U_{1}\right) / \mathscr{C}\left(U_{0}\right) \oplus \zeta^{k} \mathcal{C}\left(U_{1}\right)$. Thus, $H^{1}\left(T \mathbb{P}_{1}, \mathcal{C}(k)\right)$ is infinite dimensional. With respect to the covering by $U_{0}, U_{1}$ the cocycles $\eta^{i} / \zeta^{j}, i \geqq 0$, $j \in\{1, \ldots, 2 i-k-1\}$ respect non-zero elements in $H^{1}\left(T \mathbb{P}_{1}, O(2 k)\right)$ which are all linearly independent.

Proof. a) is the result of explicit computation, using the transition function from $U_{1}$ to $U_{0}$.

b) is proven in [Hi2], p. 164.

c) follows from the fact that $U_{0}, U_{1}$ form a Leray cover of $T \mathbb{P}_{1}$.

We now can recall results from [M], specialised here to the case of $S U(N)$. One has, as $r \rightarrow \infty$, that $\Phi=i \operatorname{diag}\left(\mu_{1}, \mu_{2}, \ldots, \mu_{N}\right)-i \operatorname{diag}\left(k_{1}, \ldots, k_{N}\right) / 2 r+O\left(r^{-2}\right)$; as $\mu_{1}>\mu_{2}>\cdots>\mu_{N}$, this implies that along each line, for each $p \in\{1, \ldots, N\}$, there is a $p$-dimensional subspace $E_{p}^{+}$of solutions to $\left(\nabla_{u}-i \Phi\right)_{S}=0$ which are bounded by const. $\exp \left(-\mu_{p} r^{r} r^{\left(k_{p}\right)}\right.$ as $r \rightarrow \infty$. This defines a flag $0 \subset E_{1}^{+} \subset \cdots \subset E_{N-1}^{+} \subset E$, which varies holomorphically; or, in other terms, a reduction of the structure group 
of $E$ from $S L(N, \mathbb{C})$ to a Borel (e.g. upper triangular) subgroup. Considering boundary behaviour as $r \rightarrow-\infty$ along each oriented line similarily gives a flag $0 \subset E_{1}^{-} \subset E_{2}^{-} \subset \cdots \subset E_{N-1}^{-} \subset E$. The real structure on $E$ maps $E_{p}^{ \pm}$to the annihilator of $E_{N-p}^{\mp}$. Moreover, one has the identifications:

$$
\begin{gathered}
E_{1}^{+} \cong L^{\mu_{1}}\left(-k_{1}\right), \\
0 \rightarrow E_{1}^{+} \rightarrow E_{2}^{+} \rightarrow L^{\mu_{2}}\left(-k_{2}\right) \rightarrow 0, \\
0 \rightarrow E_{2}^{+} \rightarrow E_{3}^{+} \rightarrow L^{\mu_{3}}\left(-k_{3}\right) \rightarrow 0, \\
\quad 0 \rightarrow E_{N-1}^{+} \rightarrow E \rightarrow L^{\mu_{N}}\left(-k_{N}\right) \rightarrow 0, \\
E_{1}^{-} \cong L^{\mu_{N}}\left(k_{N}\right), \\
0 \rightarrow E_{1}^{--} \rightarrow E_{2}^{-} \rightarrow L^{\mu_{N-1}}\left(k_{N-1}\right) \rightarrow 0, \\
0 \rightarrow E_{2}^{-} \rightarrow E_{3}^{-} \rightarrow L^{\mu_{N-2}}\left(k_{N-2}\right) \rightarrow 0, \\
-E_{N-1}^{-} \rightarrow E \rightarrow L^{\mu_{1}}\left(k_{1}\right) \rightarrow 0 .
\end{gathered}
$$

In $[\mathrm{M}]$, it is shown that for $(\zeta, \eta)$ generic, and in particular for $(\zeta, \eta)$ outside a compact set, these two flags are transversal, i.e. $E_{p}^{+} \cap E_{N-p}^{-}=\{0\}$ for all $p$. It is the set where these are not transversal, however, which is important.

Definition 1.4. The $\mathrm{p}^{\text {th }}$ spectral curve $S_{p}(p \in\{1 \cdots N-1\})$ of the monopole is defined by the vanishing of the map $\wedge^{p} E_{p}^{+} \rightarrow \wedge^{p}\left(E / E_{N-p}^{-}\right)$.

Remarks. 1) From (1.3), $\wedge{ }^{p} E_{p}^{+}$is $L^{\mu_{1}+\cdots+\mu_{p}}\left(-k_{1}-\cdots-k_{p}\right)$, and $\wedge^{p}\left(E_{/}^{\prime} E_{N-p}^{-}\right)$ is $L^{\mu_{1}+\cdots+\mu_{p}}\left(k_{1}+\cdots+k_{p}\right) . S_{p}$ is therefore a curve in the linear system $\left|0\left(2 m_{p}\right)\right|$, where $m_{p}=k_{1}+\cdots+k_{p}$ is the $p^{\text {th }}$ magnetic charge. As noted above, $S_{p}$ is compact; it can thus be given the equation

$$
g_{p}(\eta, \zeta)=\eta^{m_{p}}+a_{p, 1}(\zeta) \eta^{m_{p-1}}+\cdots+a_{p \cdot m_{p}}(\zeta)=0
$$

where the $a_{p, i}$ are of degree $2 i$.

2) An alternative definition of $S_{p}$ is by the vanishing of $\wedge^{N-p}\left(E_{N-p}^{-}\right) \rightarrow$ $\wedge^{N-p}\left(E / E_{p}^{+}\right)$. As a set, $S_{p}$ is the locus where $E_{p}^{+} \cap E_{N-p}^{-} \neq\{0\}$.

3) The real structure $\sigma: E \rightarrow E^{*}$ maps $E_{p}^{+}$at $(\eta, \zeta)$ to the annihilator $\left(E_{N-p}^{-}\right)^{\perp}$ of $E_{N-p}^{-}$at $\tau(\eta, \zeta) ; E_{N-p}^{-}$is mapped to $\left(E_{p}^{+}\right)^{\perp}$. However $E_{p}^{+} \cap E_{N-p}^{-} \neq\{0\} \Leftrightarrow$ $\left(E_{p}^{+}\right)^{\perp} \cap\left(E_{N-p}^{-}\right)^{\perp} \neq\{0\}$; therefore $S_{p}$ is real (preserved by $\tau$ ).

From (1.3) there is an exact sequence

$$
0 \rightarrow \wedge{ }^{p} E_{p}^{+} \rightarrow \wedge{ }^{p} E_{p+1}^{+} \rightarrow \wedge{ }^{p-1} E_{p}^{+} \otimes L^{\mu_{p+1}}\left(-k_{p+1}\right) \rightarrow 0 .
$$

The map $\wedge^{p} E_{p+1}^{+} \rightarrow \wedge^{p}\left(E / E_{N-p}^{-}\right)$then passes to the quotient over $S_{p}$; there is over $S_{p}$ a well defined map $\wedge^{p-1} E_{p}^{+} \otimes L^{\mu_{p+1}}\left(-k_{p+1}\right) \rightarrow \wedge^{p}\left(E / E_{N-p}^{-}\right)$. Restrict this to $\wedge^{p-1}\left(E_{p-1}^{+}\right) \otimes L^{\mu_{p+1}}\left(-k_{p+1}\right)$; using (1.3), the restricted map gives an element $\rho_{p}$ of $H^{0}\left(S_{p}, L^{\mu_{p}-\mu_{p+1}}\left(m_{p-1}+m_{p+1}\right)\right)$. In a similar vein, one obtains from the map $\wedge^{N-p-1}\left(E_{N-p-1}^{-}\right) \otimes L^{\mu_{p+1}}\left(k_{p+1}\right) \rightarrow \wedge^{N-p}\left(E / E_{p}^{+}\right)$over $S_{p}$ an element $\zeta_{p}$ of $H^{0}\left(S_{p}, L^{\mu_{p+1}-\mu_{p}}\left(m_{p-1}+m_{p+1}\right)\right)$.

Another way of defining the spectral curves is as follows. Choose a trivialisation 
of $E$ in which the positive flag $E_{i}^{+}$is mapped to the standard flag in $\mathbb{C}^{n}$. The negative flag $E_{i}^{-}$then defines, locally, a map from $T \mathbb{P}_{1}$ into the flag manifold. The spectral curves are then the pull-backs of the closures of the codimension one Bruhat cells. In a similar fashion, the intersections of the spectral curves are the pull-backs of the closures of the codimension two cells; in particular, the intersection of the curves $S_{p}$ and $S_{p+1}$ corresponds to the closure of two codimension two cells; correspondingly, one can write $S_{p} \cap S_{p+1}$ as the union of two pieces:

$$
\begin{gathered}
S_{p, p+1}: \operatorname{dim}\left(E_{p}^{+} \cap E_{N-p-1}^{-}\right) \geqq 1, \\
S_{p+1, p}: \operatorname{dim}\left(E_{p+1}^{+} \cap E_{N-p}^{-}\right) \geqq 2 .
\end{gathered}
$$

It is easy to check that they are interchanged by the real structure.

We now discuss genericity. If one refers to Taubes' construction of $S U(N)$ monopoles [T1], one finds that they are obtained there from approximate solutions which are the superposition of $\Sigma m_{p}$ well separated $S U(2)$-monopoles of charge 1. These have spectral curves which are real lines $C_{\lambda}$ in $T \mathbb{P}_{1}$. Furthermore, as in [AHi, Proposition 3.10; M], the spectral curves of the monopole one obtains approximate this union of lines; this approximation improves with the separation of the $S U(2)$ monopoles. As the $C_{x}$ can be chosen with well separated distinct intersections, one obtains monopoles whose spectral curves intersect in distinct points.

Our generic monopoles are then those for which $S_{p} \cap S_{p+1}$ consists of $2 m_{p} m_{p+1}$ distinct points, for $p=1, \ldots, N-1$. When $S_{p} \cap S_{p+1}$ have no common components, we have the lemma:

Lemma 1.8[M]. Over $S_{p}$

a) The divisor $S_{p-1, p}+S_{p, p-1}$ is cut out by $S_{p-1}$, and therefore is in the linear system $\left|O\left(2 m_{p-1}\right)\right| ;$ similarly, $S_{p, p+1}+S_{p+1, p}$ is cut out by $S_{p+1}$, and is in the linear system $\left|\mathcal{O}\left(2 m_{p+1}\right)\right|$.

b) The divisor of $\xi_{p}$ in $H^{0}\left(S_{p}, L^{\mu_{p+1}-\mu_{p}}\left(m_{p-1}+m_{p+1}\right)\right)$ is $S_{p, p-1}+S_{p, p+1}$; that of $\rho_{p}$ is $S_{p-1, p}+S_{p+1, p} . \xi_{p}, \rho_{p}$ are interchanged by the real structure.

If $[D]$ refers to the line bundle corresponding to a divisor $D$, then the fact that $L^{\mu_{\mu+1} \cdots \mu_{p}}\left(m_{p-1}+m_{p+1}\right) \cong\left[S_{p, p-1}+S_{p, p+1}\right]$ over $S_{p}$ imposes non-trivial constraints on the curve. This is condition $C-1$

From this lemma, we find by computing degrees that $S_{p . p+1}$ and $S_{p+1, p}$ both consist of $m_{p} m_{p+1}$ points, and so in the generic case:

$$
S_{p . p+1} \text { and } S_{p+1, p} \text { are disjoint. }
$$

This has several consequences:

$$
\begin{aligned}
\operatorname{dim}\left(E_{p}^{+} \cap E_{N-p-1}^{-}\right) & =1 \text { on } S_{p, p+1}, \quad 0 \text { elsewhere, } \\
\operatorname{dim}\left(E_{p}^{+} \cap E_{N-p}^{-}\right)=1 & \text { on } S_{p}^{*}, \quad 0 \text { elsewhere. }
\end{aligned}
$$

1b) A Meromorphic Reduction to a Torus. Both the positive and the negative flags define reductions of the structure group of $E$ from $S L(N, \mathbb{C})$ to Borel subgroups. Away from the spectral curves, the two flags are transversal, and define holomorphic reductions to a (complex) torus (i.e. a Cartan subgroup): $E$ can be thought of as 
a sum of line bundles $E /\left(E_{p}^{+}+E_{N-p-1}^{-}\right)$. This reduction fails over the spectral curves; this is why we refer to the reduction as "meromorphic." This failure encodes the essential structure of $E$. Before examining this, we first study the structure of certain quotient sheaves of $E$, when $E$ is generic.

i) $E /\left(E_{p}^{+}+E_{N-p}^{-}\right)$: This sheaf is concentrated over $S_{p}$, Its structure can be obtained as follows. Using the genericity conditions (1.9), one sees that $\operatorname{dim}\left(E_{p}^{+} \cap\right.$ $\left.E_{N-p}^{-}\right)=1$ over $S_{p}$; the same is then true of $E /\left(E_{p}^{+}+E_{N-p}^{-}\right)$and the sections of $E /\left(E_{p}^{+}+E_{N-p}^{-}\right)$form a locally free rank one sheaf over $S_{p}$. Next, from the exact sequence $0 \rightarrow E_{p}^{+} \rightarrow E_{p+1}^{+} \rightarrow L^{\mu_{p+1}}\left(-k_{p+1}\right) \rightarrow 0$, one sees that the natural $\operatorname{map} E_{p+1}^{+} \rightarrow E /\left(E_{p}^{+}+E_{N-p}^{-}\right)$factors through $L^{\mu_{p+1}}\left(-k_{p+1}\right)$. Referring to (1.7), this is zero precisely at $S_{p+1, p}$. Therefore, over $S_{p}, E /\left(E_{p}^{+}+E_{N-p}^{-}\right) \cong L^{\mu_{p+1}}\left(-k_{p+1}\right)$ $\left[S_{p+1, p}\right]$. Referring to (1.8), this can be written as:

$$
E /\left(E_{p}^{+}+E_{N-p}^{-}\right) \cong L^{\mu_{p+1}}\left(m_{p}+m_{p+1}\right)\left[-S_{p, p+1}\right] .
$$

Similarily, one can get

$$
E /\left(E_{p}^{+}+E_{N-p}^{-}\right) \cong L^{\mu_{p}}\left(m_{p-1}+m_{p}\right)\left[-S_{p-1, p}\right]
$$

ii) $E /\left(E_{p}^{+}+E_{N-p-1}^{-}\right)$: One first notes that $E_{p}^{+} \cap E_{N-p-1}^{-}=\{0\}$, except over $S_{p, p+1}$, where $\operatorname{dim} E_{p}^{+} \cap E_{N-p-1}^{-}=1$, by genericity. The quotient $Q=E /$ $\left(E_{p}^{+}+E_{N-p-1}^{-}\right)$is then free (a line bundle) away from $S_{p, p+1}$. We first examine its global structure, then consider the local structure near $S_{p, p+1}$. From the exact sequence of sheaves

$$
0 \rightarrow E_{p}^{+} \oplus E_{N-p-1}^{-} \rightarrow E \rightarrow Q \rightarrow 0,
$$

one has an injection of sheaves $\wedge^{N-1}\left(E_{p}^{+} \oplus E_{N-p-1}^{-}\right) \otimes Q \rightarrow \wedge^{N} E$. Referring to (1.3), this yields an injection $Q \rightarrow L^{\mu_{p+1}}\left(m_{p}+m_{p+1}\right)$, which is an isomorphism away from $S_{p, p+1}$. Locally, this can be thought of as an injection $Q \rightarrow \mathcal{O}$, the sheaf of functions of $T \mathbb{P}_{1} ; Q$ is an ideal sheaf. We now show that in fact $Q$ is locally the ideal sheaf $\mathscr{I}\left(S_{p, p+1}\right)$ of $S_{p, p+1}$. Let $v_{1}^{+}$be a section of $E_{p}^{+}, v_{1}^{-}$a section of $E_{N-p-1}^{-}$, with $v_{1}^{+}=v_{1}^{-}$at a point $x$ of $S_{p, p+1}$. We complete to local bases:

$$
\begin{array}{ll}
v_{1}^{+}, \ldots, v_{p}^{+} \text {of } E_{p}^{+}, & v_{1}^{-} \cdots v_{N-p-1}^{-} \text {of } E_{N-p-1}^{-}, \\
v_{1}^{+}, \ldots, v_{p}^{+}, v_{p+1}^{+} \text {of } E_{p+1}^{+}, & v_{1}^{-} \cdots v_{N-p-1}^{-}, v_{N-p}^{-} \text {of } E_{N-p}^{-},
\end{array}
$$

by genericity, as $S_{p+1, p}$ is disjoint from $S_{p, p+1}, E$ is the sum of $E_{p+1}^{+}$and $E_{N-p}^{-}$near $x$. One has:

at $x: v_{p+1}^{+}, v_{N-p}^{-} \operatorname{span} E /\left(E_{p}^{+}+E_{N-p-1}^{-}\right)$

along $S_{p} \backslash\{x\}: v_{p+1}^{+}$spans $E /\left(E_{p}^{+}+E_{N-p-1}^{-}\right) \cong E /\left(E_{p}^{+}+E_{N-p}^{-}\right)$, but $v_{N-p}^{-}$maps to zero.

along $S_{p+1} \backslash\{x\}: v_{N-p}^{-}$spans $E /\left(E_{p}^{+}+E_{N-p-1}^{-}\right) \cong E /\left(E_{p+1}^{+}+E_{N-p-1}^{-}\right)$, but $v_{p+1}^{+}$ maps to zero.

One can in fact choose local defining equations $a=0, b=0$ for $S_{p}, S_{p+1}$, so that locally one has the exact sequence

$$
\begin{aligned}
0 \rightarrow \mathcal{O} & \rightarrow \quad \mathcal{O}^{\oplus 2} \rightarrow Q \rightarrow 0 . \\
f & \mapsto(a f, b f) \\
& (s, t) \mapsto\left[s v_{p+1}^{+}+t v_{N-p}^{-}\right] .
\end{aligned}
$$


This is, however, precisely the form of the Koszul resolution of $\mathscr{I}\left(S_{p, p+1}\right)$ :

$$
\begin{gathered}
0 \rightarrow \mathcal{O} \rightarrow \quad \mathcal{O}^{\oplus 2} \rightarrow \mathscr{I}\left(S_{p, p+1}\right) \rightarrow 0 \\
f \mapsto(a f, b f) \\
(s, t) \mapsto(s b-t a),
\end{gathered}
$$

and so, locally, $Q \cong \mathscr{I}\left(S_{p, p+1}\right)$. Therefore, globally,

$$
E /\left(E_{p}^{+}+E_{N-p-1}^{-}\right) \cong L^{\mu_{p+1}}\left(m_{p}+m_{p+1}\right) \otimes \mathscr{I}\left(S_{p, p+1}\right) .
$$

We now give a description of $E$ valid for any monopole bundle.

Proposition 1.12. One has the short exact sequence of sheaves:

$$
\begin{aligned}
& \begin{array}{lll}
E / E_{N-1}^{-} & \begin{array}{l}
\oplus \\
\oplus /\left(E_{1}^{+}+E_{N-2}^{-}\right) \\
\oplus
\end{array} & E /\left(E_{1}^{+}+E_{N-1}^{-}\right) \\
\oplus & \begin{array}{c}
\oplus \\
\oplus
\end{array}
\end{array}
\end{aligned}
$$

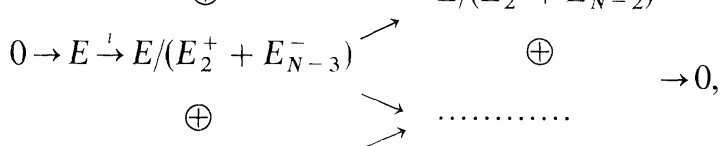

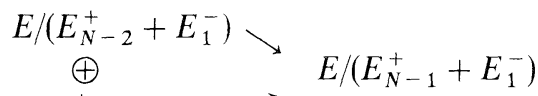

$$
\begin{aligned}
& E / E_{N-1}^{+}
\end{aligned}
$$

where the map between the second and third terms is of the form $\left(a_{1} \cdots a_{N}\right) \mapsto\left(a_{1}-a_{2}\right.$, $\left.a_{2}-a_{3}, \ldots, a_{N-1}-a_{N}\right)$.

Proof. The only non-trivial part is showing that $\operatorname{ker} \pi \subset \operatorname{Im} i$, i.e. that $\pi=0$ imposes sufficient constraints on a section of the middle term for it to come from $E$. Let $\left(x_{i}+\left(E_{i}^{+}+E_{N-i}^{-}\right)\right) i=0, \ldots, N-1$ represent a local section in $\operatorname{ker} \pi$. One wants a section $y$ of $E$ such that

$y+\left(E_{i}^{+}+E_{N-i-1}^{-}\right)=x_{i}+\left(E_{i}^{+}+E_{N-i-1}^{-}\right)$for all $i$, and so $y \in \bigcap_{i=0}^{N-1} x_{i}+\left(E_{i}^{+}+E_{N-1}^{-}\right)$.

Now if $A, B$ are subsheaves of $E$, one has the sequence

$$
0 \rightarrow E /(A \cap B) \rightarrow E / A \oplus E / B \rightarrow E /(A+B) \rightarrow 0 .
$$

As $x_{0}, x_{1}$ map to the same element in $E /\left(E_{1}^{+}+E_{N-1}^{-}\right)$, there is a $y_{1}+$ $\left(E_{N-1}^{-} \cap\left(E_{1}^{+}+E_{N-2}^{-}\right)\right)$mapping to both $x_{0}+E_{N-1}^{-}$and $x_{1}+\left(E_{1}^{+}+E_{N-2}^{-}\right)$. The problem is now to find $y \in\left(\bigcap_{i=2}^{N-1} x_{i}+\left(E_{i}^{+}+E_{N-i-1}^{-}\right)\right) \cap\left(y_{1}+\left(E_{N-1}^{-} \cap\right.\right.$ $\left.\left.\left(E_{1}^{+}+E_{N-2}^{-}\right)\right)\right)$. As $E_{N-2}^{-} \subset E_{N-1}^{-} \cap\left(E_{1}^{+}+E_{N-2}^{-}\right)$, it suffices to find $y \in$ $\left(\bigcap_{i=2}^{N-1} x_{i}+\left(E_{i}^{+}+E_{N-i-1}^{-}\right)\right) \cap\left(y_{1}+E_{N-2}^{-}\right)$. Now, by hypothesis, $y_{1}$ and $x_{2}$ map to the same element in $E /\left(E_{2}^{+}+E_{N-2}^{-}\right)$; proceeding as above, there is a $y_{2}+\left(E_{N-2}^{-} \cap\left(E_{2}^{+}+E_{N-3}^{-}\right)\right)$mapping to both $y_{1}+E_{N-2}^{-}$and $x_{2}+\left(E_{2}^{+}+E_{N-3}^{-}\right)$, and 
the problem then reduces to find $y \in\left(\bigcap_{i=3}^{N-1} x_{i}+\left(E_{i}^{+}+E_{N-i-1}^{-}\right)\right) \cap\left(y_{2}+E_{N-3}^{-}\right)$. Iterating this procedure, one obtains $y_{3}, y_{4}, \ldots$, and $y_{N-1}=y$.

Using the identifications of the quotients given above, we therefore see that in the generic case $E$ fits into an exact sequence:

$$
\begin{aligned}
& L^{\mu_{1}}\left(m_{1}\right) \\
& \left.\stackrel{\oplus}{L^{\mu_{2}}\left(m_{1}+m_{2}\right) \otimes \mathscr{I}\left(S_{1,2}\right)} \longrightarrow L^{\mu_{1}}\left(m_{1}\right)\right|_{S_{1}} \\
& \left.\oplus \longrightarrow L^{\mu_{2}}\left(m_{1}+m_{2}\right)\left[-S_{1.2}\right]\right|_{S_{2}} \\
& 0 \rightarrow E \rightarrow L^{\mu_{3}}\left(m_{2}+m_{3}\right) \otimes \mathscr{I}\left(S_{2,3}\right) \succ \ldots \ldots \ldots \ldots \ldots \ldots . .
\end{aligned}
$$

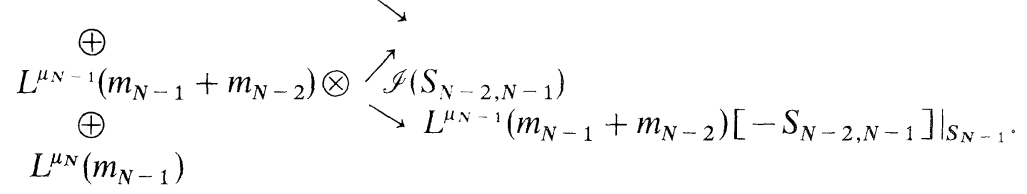

Let $r_{p}$ denote restriction to the $p^{\text {th }}$ spectral curve, and, referring to (1.8) and (1.5), let $f_{p}=\rho_{p} g_{p+1}^{-1}$ be a meromorphic section over $S_{p}$ of $L^{\mu_{p}-\mu_{p+1}}\left(m_{p-1}-m_{p+1}\right)$, which has poles at $S_{p, p+1}$ and zeroes at $S_{p-1, p}$. The map between the second and third terms above is:

$$
\begin{aligned}
\Pi\left(a_{1}, \ldots, a_{N}\right)= & \left(r_{1}\left(a_{1}\right)-f_{1} r_{1}\left(a_{2}\right), r_{2}\left(a_{2}\right)-f_{2} r_{2}\left(a_{3}\right), \ldots, r_{N-1}\left(a_{N-1}\right)\right. \\
& \left.-f_{N-1} r_{N-1}\left(a_{N}\right)\right) .
\end{aligned}
$$

We call the curves $S_{p}$ and the splitting $S_{p} \cap S_{p+1}=S_{p, p+1} \cup S_{p+1, p}$ the spectral data of the monopole. As the divisors $S_{p, p+1}, S_{p-1, p}$ determine the sections $f_{p}$, we have from (1.14) a result from [M]:

Proposition 1.15. A generic monopole is determined by its spectral data.

Remark 1.16. Note that one can also interpret the flag structure of $E$ in terms of (1.12). Local sections of $E_{p}^{+}$are elements in the kernel of $\Pi$ of the form $\left(a_{1}, \ldots, a_{p}\right.$, $0, \ldots, 0)$. This forces $a_{p}$ not only to vanish on $S_{p-1, p}$, but on the whole of $S_{p} ; a_{p}$ is then a local section of $L^{\mu_{p}}\left(m_{p-1}-m_{p}\right)=L^{\mu_{p}}\left(-k_{p}\right)$. From this, one can reobtain the extensions

$$
0 \rightarrow E_{p-1}^{+} \rightarrow E_{p}^{+} \rightarrow L^{\mu_{p}}\left(-k_{p}\right) \rightarrow 0 .
$$

Similarly, local sections of $E_{N-p}^{-}$are of the form $\left(0, \ldots, 0, a_{N-p+1}, \ldots, a_{N}\right)$. One also notes that one has exact sequences, for any monopole

$$
\begin{aligned}
& 0 \rightarrow L^{\mu_{p}}\left(-k_{p}\right) \rightarrow E /\left(E_{p-1}^{+}+E_{N-p}^{-}\right) \rightarrow E /\left(E_{p}^{+}+E_{N-p}^{-}\right) \rightarrow 0, \\
& 0 \rightarrow L^{\mu_{p}}\left(k_{p}\right) \rightarrow E /\left(E_{p-1}^{+}+E_{N-p}^{-}\right) \rightarrow E /\left(E_{p-1}^{+}+E_{N-p+1}^{-}\right) \rightarrow 0 .
\end{aligned}
$$

\section{C) A Vanishing Theorem.}

Theorem 1.17. Let $S_{p}$ be the $p^{\text {th }}$ spectral curve of a generic monopole. Then $W_{z}=$ $H^{0}\left(S_{p}, L^{-z}(-2) \otimes E /\left(E_{p}^{+}+E_{N-p}^{-}\right)\right)=0$. (Generically, 


$$
\left.W_{z}=H^{0}\left(S_{p}, L^{\mu_{p}-z}\left(m_{p}+m_{p-1}-2\right)\left[-S_{p-1, p}\right]\right) .\right)
$$

a) $\forall z \in\left(\mu_{p+1}, \mu_{p}\right)$.

b) For $z=\mu_{p}$, if $m_{p} \leqq m_{p-1} \cdot(1<p)$.

c) For $z=\mu_{p+1}$, if $m_{p} \leqq m_{p+1} \cdot(p<N)$.

Proof. The idea is to use (1.12). By a coboundary map $\delta$, the space $W_{z}$ above maps into $H^{1}\left(T \mathbb{P}_{1}, E L^{-z}(-2)\right)$. This, in turn, by the twistor transform, corresponds to solutions to a Laplace type equation over $\mathbb{R}^{3}$. By computing boundary behaviour of solutions in $\delta\left(W_{z}\right)$, we show that they must vanish.

We start by showing that $\delta$ is an injection. An element $s$ of $W_{z}$ can be thought of as a section $(0, \ldots, 0, s, 0 \cdots 0)$ of the right-hand term of $(1.12)$ (twisted by $L^{-z}(-2)$ ). If it maps to zero in $H^{1}\left(T \mathbb{P}_{1}, E L^{-z}(-2)\right)$, then it is the image of a section of the middle term of (1.12). Let us first consider the generic $E$. For these, in case a), the middle term has only the zero section, as by $(1.2), H^{0}\left(T \mathbb{P}_{1}, L^{t}(k)\right)=0 \forall k, \forall t \neq 0$, and so $s=0$. In case b), consider the following portion of $(1.14) \otimes L^{\mu_{p}}(-2)$ :

$$
\begin{aligned}
& D=L^{\mu_{p-1}-\mu_{p}}\left(m_{p-2}+m_{p-1}-2\right) \otimes \mathscr{I}\left(S_{p-2, p-1}\right) \\
& C=\mathcal{O}\left(m_{p}+m_{p-1}-2\right) \otimes \mathscr{I}\left(S_{p-1, p}\right) \\
& \searrow \\
& \qquad=L^{\mu_{p+1}-\mu_{p}}\left(m_{p+1}+m_{p}-2\right) \otimes \mathscr{I}\left(S_{p, p+1}\right) .
\end{aligned}
$$

For the section $(0, s)$ of $(B, A)$ that we are considering to be in the image of the left-hand side, one must have sections $(d, c, e)$ of $(D, C, E)$ with $(0, s)=\left(r_{p-1}(d)-\right.$ $\left.f_{p-1} r_{p-1}(c), r_{p}(c)-f_{p} r_{p}(e)\right)$. However, by (1.2), $d=e=0$. Therefore $e$ vanishes on $S_{p-1}$, and so can be thought of as a section of $\mathcal{O}\left(m_{p}-m_{p-1}-2\right)$. By hypothesis, this has negative degree; therefore $c=0$, and so $s=0$. Injectivity for the third case of the theorem is proven in a similar fashion.

When $E$ is non-generic, the injectivity is proven in essentially the same fashion but is notationally more complicated: one uses the sequences of (1.16) to express the lifts to the middle portion of $\left((1.12) \otimes L^{-z}(-2)\right.$, of sections of the right-hand side of the form $(0,0, \ldots, 0, s, 0, \ldots, 0)$ in terms of sections of $\oplus_{i} L^{\mu_{i}-z}\left(k_{i}-2\right)$, which must vanish.

Having shown that $\delta$ is injective, we compute Cech and Dolbeault representatives for $\delta(s)$. Let $V_{i}, i=0, \ldots, n$ be a sufficiently fine covering of $T \mathbb{P}_{1}$ with $V_{i}$, $i==1, \ldots, n$ covering the spectral curves, $\bigcup_{i=1}^{n} V_{i}$ lying inside a compact set, and $V_{0}$ not intersecting the spectral curves. Over each $V_{i}$, one can pull back the section $(0,0, \ldots, 0, s, 0 \cdots 0)$ to the middle term of $(1.12) \otimes L^{-z}(-2)$, in two particularly convenient ways:

1) as a local section $f_{1}^{+}$of $E_{p}^{+}$, of the form $\left(f_{i, 1}^{+}, \ldots, f_{i, p}^{+}, 0, \ldots, 0\right)$,

(2) as a local section $f_{i}^{-}$of $E_{N-p}^{-}$, of the form $\left(0, \ldots, 0, f_{i, p+1}, \ldots, f_{i, N}^{-}\right)$. 
Setting $f_{0}^{ \pm}=0, \delta(s)$ then has two representative cocycles $f_{ \pm}$defined by $f_{i j}^{ \pm}=$ $f_{i}^{ \pm}-f_{j}^{ \pm}$over $V_{t} \cap V_{j}$, differing of course by a coboundary; $f_{+}$represents an element of $H^{1}\left(T \mathbb{P}_{1}, E_{p}^{+} L^{-z}(-2)\right), f_{-}$, an element of $H^{1}\left(T \mathbb{P}_{1}, E_{N-p}^{-} L^{-Z}(-2)\right)$.

Dolbeault representatives are obtained in a standard way. If $\sigma_{i}$ is a partition of unity subordinate to $V_{i}$, set $\theta_{j}^{ \pm}=\bar{\partial}\left(\sum_{i} \sigma_{i} f_{j i}^{ \pm}\right) ; \theta_{j}^{ \pm}=\theta_{k}^{ \pm}$on overlaps, and so one gets globally defined forms $\theta^{+} \in \Omega^{0,1}\left(E_{p}^{+} L^{-z}(-2)\right), \theta^{-} \in \Omega^{0,1}\left(E_{N-p}^{-} L^{-z}(-2)\right)$, both representing $\delta(s)$. Furthermore, if $\gamma=\sum_{i} \sigma_{i}\left(f_{i}^{+}-f_{i}^{-}\right), \theta^{+}-\theta^{-}=\bar{\partial} \gamma$. Note that as $\mathcal{O}(-2) \simeq \Pi^{*}\left(K\left(\mathbb{P}_{1}\right)\right)\left(\Pi: T \mathbb{P}_{1} \rightarrow \mathbb{P}_{1}\right), \theta^{ \pm}$can be considered as $(1,1)$ forms with values in $E L^{-z}$; these forms have terms in $d \zeta \wedge d \bar{\eta}, d \zeta \wedge d \bar{\zeta}$ and none in $d \eta \wedge d \bar{\zeta}, d \eta \wedge d \bar{\eta}$; furthermore, $\theta^{+}, \theta^{-}$and $\gamma$ are all compactly supported in some disk bundle $D$ inside $T \mathbb{P}_{1}$.

The proof is now a slightly refined version of that found in Hitchin, [Hi2, p. 162] (see also $[\mathrm{HiM}]$ ). Recall the twistor transform over $\mathbb{R}^{3}$. First, the bundle $H$ over $\mathbb{R}^{3}$ is reobtained by $H_{x}=H^{0}\left(C_{x}, E L^{-z}\right)$. Secondly, as $E L^{-z}$ trivial over $C_{x}$, $H^{1}\left(C_{x}, E L^{-z}(-2)\right) \simeq H^{0}\left(C_{x}, E L^{-z}\right) \otimes H^{1}\left(C_{x}, \mathcal{O}(-2)\right)$. By Serre duality (integrating a representative $(1,1)$ form $), H^{1}\left(C_{x}, \mathcal{O}(-2)\right) \simeq \mathbb{C}$. Thus, restricting our element $\delta(s)$ to $C_{x}$, we get an element $F(x)$ of $H_{x} . F$ is in fact the solution to a Laplace type equation over $\mathbb{R}^{3}$; the map from $H^{1}\left(T \mathbb{P}_{1}, E L^{-z}(-2)\right)$ to the space of such solutions is bijective.

We examine the behaviour of our solution at a point $x$ of $\mathbb{R}^{3}$ as $x \rightarrow \infty$. For concreteness, take $x$ to be the point $(0,0,-b / 2)$; then $C_{x}$ is defined by $\eta=b \zeta$. The intersection of $C_{x}$ with $D$, for $|x|$ large, is the disjoint union of two open sets $A_{x}^{+}$, $A_{x}^{-}$centred around $\zeta=0, \zeta=\infty$ respectively on $C_{x}$. Note that their radii (in $\zeta$ ) tend to zero linearily in $b^{-1}$ as $b \rightarrow \infty$.

Let $\theta^{ \pm}$be written locally as $\alpha^{ \pm} d \zeta \wedge d \bar{\eta}+\beta^{ \pm} d \zeta \wedge d \bar{\zeta}$; let their restrictions to $C_{x}$ be $\sum_{i} s_{i}\left(\alpha_{i}^{ \pm} d \zeta \wedge d \bar{\eta}+\beta_{i}^{ \pm} d \zeta \wedge d \bar{\zeta}\right)=\sum_{i} s_{i}\left(b \alpha_{i}^{ \pm}+\beta_{i}^{ \pm}\right) d \zeta \wedge d \bar{\zeta}$, where the $s_{l}$ are an orthonormal basis of the sections of $E$ over $C_{x}$. Similarly, set $\gamma$ over $C_{x}$ to be $\Sigma s_{i}\left(\rho_{i} d \zeta\right)$. One has, over $C_{x},\left[\left(b \alpha_{i}^{+}+\beta_{i}^{+}\right)-\left(b \alpha_{i}^{-}+\beta_{i}^{-}\right)\right] d \zeta \wedge d \bar{\zeta}=\bar{\partial}\left(\rho_{i} d \zeta\right)=d\left(\rho_{i} d \zeta\right)$. The coefficient of $s_{i}$ in $F(x)$ is:

$$
\int_{C_{x}}\left(b \alpha_{i}^{+}+\beta_{i}^{+}\right) d \zeta \wedge d \bar{\zeta}=\int_{A_{2}^{+}}\left(b \alpha_{i}^{+}+\beta_{i}^{+}\right) d \zeta \wedge d \bar{\zeta}+\int_{A_{x}^{-}}\left(b \alpha_{i}^{-}+\beta_{i}^{-}\right) d \zeta \wedge d \bar{\zeta}
$$

using Stokes' theorem.

We now use the fact that $\theta^{+} \in \Omega^{1,1}\left(E_{p}^{+} L^{-z}\right)$. Elements of $E_{p}^{+} L^{-z}$ at a point of $T \mathbb{P}_{1}$ correspond to solutions of $\left(\nabla_{u}-i \Phi\right) s=0$ which are bounded by const. $\left[\exp \left(-\left(\mu_{p}-z\right) b\right) b^{-\left(k_{p}\right)}\right]$ as $b \rightarrow \infty$. Following Hitchin [Hi2, p. 163], this means that the coefficients $\alpha_{i}^{+}, \beta_{i}^{+}$are bounded over $A_{x}^{+}$by const. $\left[\exp \left(-\left(\mu_{p}-z\right) b\right) b^{-\left(k_{p}\right)}\right]$. In the cases $a, b, c$ which interest us, the integrand is then always bounded by const. [b]; however the area of $A_{+}$is bounded by const. $\left[b^{-2}\right]$, and so the integral over $A_{x}^{+}$is bounded by const. $\left[b^{-1}\right]$. The same argument, applied to $\theta^{-} \in \Omega^{1,1}\left(E_{N-p}-L^{-z}\right)$ bounds the integral over $A_{x}^{-}$by const. $\left[b^{-1}\right]$, and so $|F(x)|$ is bounded by const. $\left[b^{-1}\right]$. As in [Hi2, p. 164], keeping track of the derivatives gives $|\nabla F(x)|$ bounded by const. $\left[b^{-2}\right]$, and so the argument given there (essentially the maximum principle) applies, forcing $F=0$. Therefore $s=0$, and the vanishing theorem is proved. 
1d) The Asymptotic Higgs Field. To begin, note that the spectral curves all lie within some compact disc bundle $D$ over $\mathbb{P}_{1}$. Then, for each $x \in \mathbb{R}^{3}$ outside a compact set $K, C_{x} \cap S_{p}$ is a set of $2 m_{p}$ points which partitions naturally into two clusters $C_{p, x}^{0}, C_{p, x}^{\infty}$ of $m_{p}$ points: the points in $C_{p, x}^{0}$ are lines in $\mathbb{R}^{3}$ through $x$ which point (approximately) away from the origin, and the points in $C_{p, x}^{\infty}$ are the same lines, but with orientation reversed, and so pointing towards the origin; $\tau\left(C_{p, x}^{0}\right)=C_{p, x}^{\infty}$.

Let $U \subset V$ be open sets in $\mathbb{P}_{1}$ representing a "cone" of directions in $\mathbb{R}^{3}$. Set $A=\left(T \mathbb{P}_{1} \backslash D\right) \cup \pi^{-1}(V) \cup \tau\left(\pi^{-1}(V)\right)$, where $\pi: T \mathbb{P}_{1} \rightarrow \mathbb{P}_{1}$ is the projection. If $|x|$ is large enough, and the line $O x$ has direction in $U$, then $C_{x} \subset A$.

Let $g_{p}=0$ be the equation defining $S_{p}$; one can define an element of $H^{1}(A, \mathcal{O}(-2))$ by the cocycle $-\left(\partial g_{p} / \partial \eta\right) /\left(2 \pi i g_{p}\right)$, relative to the covering of $A$ by $T \mathbb{P}_{1} \backslash \pi^{-1}(V), T \mathbb{P}_{1} \backslash \tau\left(\pi^{-1}(V)\right)$. This in turn, by the twistor transform [Hi3], corresponds to a solution of the (ordinary) Laplace equation, defined for the $x$ 's in $\mathbb{R}^{3}$ such that $C_{x} \subset A$. Varying $U$, these solutions patch together to give a global solution $\psi_{p}$ which is defined outside a compact set of $\mathbb{R}^{3} \cdot \psi_{p}$ has the following alternate formulations, proven in [Hu1, p. 386-387]:

Lemma (1.18).

a) Along the line in $\mathbb{R}^{3}$ corresponding to sections $\eta=b \zeta, b \rightarrow-\infty$,

$$
\psi_{p}=\partial_{b} \log \left(\prod_{\left(b \zeta_{1}, \zeta_{i}\right) \in C_{x, p}^{0}} \zeta_{i}\right)=-\partial_{b} \log \left(\prod_{\left(b \zeta_{i} \zeta_{1}\right) \in C_{x, p}^{\infty}} \zeta_{i}\right) .
$$

b) Along $C_{x}, T\left(T \mathbb{P}_{1}\right)$ splits into the sum of two canonically isomorphic bundles: $T F$, the tangents to the fibers of $T \mathbb{P}_{1} \rightarrow \mathbb{P}_{1}$. and $T C_{x}$. Assuming that $C_{x}$ intersects $S_{p}$ transversally, at smooth points of $S_{p}$; then a tangent vector $v$ to $S_{p}$ at $q \in S_{p} \cap C_{x}$ then decomposes at $\left(v_{f}, v_{c_{x}}\right)$, and the "slope" $s(q)=\left(v_{c_{x}}\right) /\left(v_{f}\right)$ of $S_{p}$ at $q$ is well defined. Then

$$
\psi_{p}(x)=\sum_{q \in C_{\lambda, p}^{0}} s(q)
$$

Theorem (1.19). Let $E$ be a vector bundle E over $T \mathbb{P}_{1}$ defined by the sequence (1.14), where the $S_{p}$ 's are real. Then,

a) For $X$ outside a compact set $K$ in $\mathbb{R}^{3}, E$ is trivial when restricted to $C_{x}$.

b) $E$ defines a Higgs field $\Phi$ and a connection $\nabla$ over $\mathbb{R}^{3} \backslash K$ such that asymptotically, the eigenvalues $\Phi_{j}$ of $\Phi$ are approximated by

$$
\left(\Phi_{j}\right)_{\mathrm{as}}=i \mu_{j}+i\left(\psi_{j}-\psi_{j-1}\right)
$$

where $\psi_{0}, \psi_{N}$ are defined to be zero. This approximation is valid up to exponentially decreasing terms with exponentially decreasing derivatives.

c) $(\nabla, \Phi)$ satisfy the boundary conditions of a monopole.

Proof. We will compute the Higgs field for $x=(0,0,-b / 2)$, as $b \rightarrow-\infty$ : this corresponds to lines $C_{x}: \eta=b \zeta$. We will suppose that $(\eta, \zeta)=(0,0) \notin S_{p}$, and so $\tau(0,0) \notin S_{p}$. This can be done without loss of generality, as for a given small cone of directions in $\mathbb{R}^{3}$, one can shift the origin so that this is true. For convenience, we will suppose that the intersection of $C_{x}$ with the curves $S_{p}$ consists of $2 m_{p}$ distinct points; the presence of multiple points does not change the proof, but, as we are using Lagrange interpolation, it does change the formulae. 
Over the line $\eta=b \zeta$, a section of $L^{\mu}(k)$ is represented over $U_{0}$ by polynomial $s_{0}$ in $\zeta$ of degree $\leqq k$, and over $U_{1}$ by a polynomial $s_{1}$ in $\zeta^{-1}$ of degree $\leqq k$, such that on the overlap:

$$
s_{0}=e^{\mu b \zeta^{k}} S_{1} .
$$

Let $C_{x}$ intersect $S_{p}$ in $2 m_{p}$ points with $\zeta$-coordinates $\zeta_{p, i}=\zeta_{p . i}(b), i=1, \ldots, 2 m_{p}$. As $C_{x}$ and $S_{p}$ are real, one can order the points so that $\zeta_{p, i} \in C_{x, p}^{0}$ (therefore $\zeta_{p, i} \rightarrow 0$ as $b \rightarrow-\infty)$ for $i=1, \ldots, m_{p}$, and so that $\zeta_{p, m_{p}+i}=-1 / \bar{\zeta}_{p, i}, i=1, \ldots, m_{p}$. In the exact sequence (1.14), meromorphic sections $f_{p}$ of $L^{\mu_{p}-\mu_{p+1}}\left(m_{p-1}-m_{p+1}\right)$ over $S_{p}$ are involved. Represent these by functions ${ }_{0} f_{p}$ over $U_{0},{ }_{1} f_{p}$ over $U_{1}$, with ${ }_{0} f_{p}=$ $\exp \left(\left(\mu_{p}-\mu_{p+1}\right) \eta / \zeta\right) \zeta^{\left(m_{p}-1-m_{p+1}\right)}{ }_{1} f_{p}$ on the overlap. Set $f_{p, j}(b)$ to be the value of ${ }_{0} f_{p}$ at $\zeta_{p, j}(b)$. Then, referring to (1.14), a section of $E$ over $C_{x}$ can be represented in the $U_{0}$ trivialisation by polynomials $s_{p}$ of degree $m_{p}+m_{p-1}\left(m_{0}=m_{N}=0\right)$ with the constraints

$$
s_{p}\left(\zeta_{p, j}\right)=f_{p, j} s_{p+1}\left(\zeta_{p, j}\right), \quad j=1 \cdots 2 m_{p}, \quad p=1 \cdots N-1 .
$$

As $b$ tends to infinity, the points $\zeta_{p, j}, j=1, \ldots, m_{p}$ tend to zero; the $\zeta_{p, m_{p}+j}$ tend to infinity, linearly in $b$. As ${ }_{1} f_{p}$ is bounded near $\zeta=\infty$, then, as $\eta=b \zeta$ on $C_{x}$, the $f_{p, m_{p}+j}$ converge to zero, exponentially in $b$. The equations (1.21) for a section are then, up to exponentially decreasing terms:

$$
\begin{aligned}
s_{p}\left(\zeta_{p, j}\right) & =f_{p, j} S_{p+1}\left(\zeta_{p, j}\right), \\
s_{p}\left(\zeta_{p, m_{p}+j}\right) & =0, \quad j=1, \ldots, m_{p} .
\end{aligned}
$$

As $b \rightarrow-\infty,(1.22)$ does not quite tend to a finite, well defined limit. However, note that $b \zeta_{p, j} \rightarrow c_{p, j}$, where $c_{p, j}$ are the $\eta$-coordinates of the points of intersection of $\{\zeta=0\}$ with $S_{p}$. We therefore set $t_{p}(b \zeta)=s_{p}(\zeta)$; if $g_{p, j}$ are the values of ${ }_{0} f_{p}$ at $\{\zeta=0\} \cap S_{p}$, then (1.22) becomes in the limit:

$$
\begin{aligned}
t_{p}\left(c_{p, j}\right)= & g_{p, j} t_{p+1}\left(c_{p . j}\right) \text {, and } t_{p} \text { has an } m_{p} \text {-fold zero at } \zeta_{o}=\infty \\
& \cdot\left(\text { i.e. } t_{p} \text { is of degree } m_{p-1}\right)
\end{aligned}
$$

For $E$ to be trivial over a line $C_{x}$, it suffices to find a basis of solutions ${ }^{i} S=\left({ }^{i} S_{p}\right)$, $i=1, \ldots, N$, to (1.22), spanning the fiber of $E$ at a point. Taking this point to be $\zeta=0$. one adds to conditions (1.22) the extra constraint

$$
{ }^{i} s_{p}(0)=\delta_{p, i}
$$

Adding this condition into the limit equations (1.23), the linear system one obtains is non-degenerate, and in fact solvable by Lagrange interpolation. Therefore, for $|b|$ large, (1.22) and (1.24) are also solvable and $E$ is trivial over $\eta=b \zeta$. By varying our coordinate system $(\eta, \zeta)$ (i.e. rotating in $\left.\mathbb{R}^{3}\right)$, one obtains part a).

We next compute the Higgs field, again along $\eta=b \zeta$. We begin with a remark: all the functions involved in this computation are of the form (in $b) \exp (-k b) \times$ (meromorphic in $b^{-1}$ ). Thus, in general terms if we obtain an exponential approximation $q^{\prime}$ of a quantity $q$, the derivatives of $q^{\prime}$ will also approximate the derivatives of $q$ exponentially.

With this in mind, we construct an exponential approximation ${ }^{i} S_{p}$ to a basis of sections of $E$ over $\eta=b \zeta$; this amounts to finding ${ }^{i} s_{p}$ solving (1.22) and (1.24), 
which is simply a matter of Lagrange interpolation. For $i$ fixed, one gets:

$$
\begin{gathered}
-{ }^{i} S_{p} \equiv 0 \text { for } p<i \\
-{ }^{p_{S}} S_{p}=\prod_{j=1}^{m_{p}-1} \frac{\left(\zeta-\zeta_{p-1, j}\right)}{\left(-\zeta_{p-1, j}\right)} \prod_{j=1}^{m_{p}} \frac{\left(\zeta-\zeta_{p, m_{p}+j}\right)}{\left(-\zeta_{p, m_{p}+j}\right)} .
\end{gathered}
$$

The ${ }^{i} s_{p}$ for $i<p$ can be computed similarily. Thus the matrix $S=\left({ }^{i} s_{p}\right)$ is lower triangular.

We then construct the asymptotic Higgs field in this basis. The family of sections $C_{x}$ all intersect at $(0,0)$ and $\tau(0,0)$. There are two natural connections on the bundle $H\left(H_{x}=H^{0}\left(C_{x}, E\right)\right)$ over the line $(0,0,-b / 2)$ in $\mathbb{R}^{3}$ : one, $\nabla_{0}$, has flat sections defined by fixing values of elements of $H_{x}$ at $(0,0)$; the other $\nabla_{\infty}$, is similarily defined at $\tau(0,0)$. The Higgs field is then [Hi1]

$$
\Phi d b=i\left(\nabla_{0}-\nabla_{\infty}\right) .
$$

The basis $S$ above is $\nabla_{0}$-constant by (1.24), and $\nabla_{0}$ then has zero matrix. To obtain the matrix of $\nabla_{\infty}$, one must first evaluate the basis at $\zeta=\infty$; taking the change of trivialisations (1.20) into account, one evaluates (in our approximation) $\left[\operatorname{diag}\left\{\exp \left(-\mu_{i} b\right) \zeta^{-\left(m_{t}+m_{2}-1\right)}\right\} \cdot S\right]$ at $\infty$, obtaining a matrix $M$. Write $M$ as $\operatorname{diag}\left(\exp \left(-\mu_{i} b\right)\right) . T ; T$ is lower triangular, with diagonal elements

$$
T_{p, p}=\prod_{j=1}^{m_{p}-1}\left(-\zeta_{p-1, j}\right)^{-1} \prod_{j=1}^{m_{p}}\left(-\zeta_{p, m_{p}+j}\right)^{-1} .
$$

The matrix of $\nabla_{\infty}$ is $\mathrm{M}^{-1}\left(\partial_{b} M\right) d b$; that of $\Phi_{\text {as }}$ is then

$$
\Phi_{\mathrm{as}}=-i M^{-1} \partial_{b} M=-i\left(T^{-1} \operatorname{diag}\left(-\mu_{i}\right) T+T^{-1} \partial_{b} T\right) .
$$

$\Phi_{\mathrm{as}}$ is lower triangular; its diagonal entries $\left(\Phi_{\mathrm{as}}\right)_{p}$ (eigenvalues) can be computed from those of $T$, to obtain

$$
\left(\Phi_{\mathrm{as}}\right)_{p}=i \mu_{p}+i \partial_{b} \log \left(\prod_{j=1}^{m_{p-1}} \zeta_{p-1, j} \prod_{j=1}^{m_{p}} \zeta_{p, m_{p}+i}\right),
$$

referring to Lemma (1.18)

$$
\left(\Phi_{\mathrm{as}}\right)_{p}=i \mu_{p}+i\left(\psi_{p}-\psi_{p-1}\right) .
$$

To prove c), one notes that

$$
\prod_{j=1}^{m_{p}-1}\left(\zeta_{p-1, j}\right) \prod_{j=1}^{m_{p}}\left(\zeta_{p, m_{p}+j}\right)
$$

has leading term $b^{m_{p}-m_{p-1}}=b^{k_{p}}$ as $b \rightarrow \infty$; this gives $\Phi_{p}=i\left(\mu_{p}-k_{p} / 2 r\right)+O\left(r^{-2}\right)$. The other estimates follow by taking derivatives as in [Hul]. One uses $|\nabla \Phi|^{2}=\Delta|\Phi|^{2} / 2$.

1e) Reality Conditions. Our monopole bundles $E$ are equipped with an antiholomorphic map $\sigma: E \rightarrow E^{*}$ lifting $\tau$. We examine how this is encoded in the spectral data; we begin by showing how to define $\sigma$ for a bundle $E$ derived from the spectral data via (1.14). Note that the two real structures $\sigma, \tau$ define an operation * from sections of $E$ to sections of $E^{*}$ by $f^{*}(p)=\sigma f(\tau p)$ for $p$ in $T \mathbb{P}_{1}$, and if $f$ is 
holomorphic so also is $f^{*}$. If $f$ and $g$ are sections of $E$ over a real section $C_{x}$, we can define a pairing by $\langle f, g\rangle=f\left(g^{*}\right)$ which on a real section is a holomorphic function, and so a constant complex number. This defines a pairing on the bundles $H$ on $\mathbb{R}^{3}\left(H_{x}=H^{0}\left(C_{x}, E\right)\right)$.

We shall show that this pairing is hermitian and positive definite, and also, that if one performs the twistor construction of the $(\nabla, \Phi)$ associated to $E$, the pairing is compatible with the connection and Higgs field. This means the monopole we have constructed is an $S U(n)$ monopole.

First let us make some normalization conventions. Every bundle $L^{\mu}(k)$ has a real structure $\sigma$ mapping $L^{\mu}(k)$ to $L^{-\mu}(k)$ and covering $\tau$. This can be chosen so that when bundles are tensored the real structures are tensored, and we shall denote all these different real structures by the same symbol. The operator * on sections of $L^{\mu}(k)$ then satisfies $* *=(-1)^{k}$. If $g_{p}$ is our normalised section of $\mathcal{C}\left(2 m_{p}\right)$ defining $S_{p}$, then we have

$$
g_{p}^{*}=g_{p} \cdot(-1)^{m_{p}} .
$$

Similarly the meromorphic sections $f_{p}$ in (1.14) are normalized to satisfy

and

$$
f_{p} f_{p}^{*}=e_{p} \frac{g_{p-1}}{g_{p+1}}, \text { where } \quad e_{p}= \pm 1
$$

$$
-(-1)^{m_{p-1}+m_{p}} e_{p}>0
$$

for all $p$ from the conditions on the spectral data. We shall see below that it is this condition on the sign of $e_{p}$ that makes the hermitian form positive definite.

We start by defining a pairing between the fibres $E(\gamma)$ and $E(\tau(\gamma))$ for any $\gamma$ in minitwistor space. Consider an open set $U$ about $\gamma$. Then a local section of $E$ over $U$, from diagram (1.14), can be regarded as a collection $s=\left(s_{1}, \ldots, s_{N}\right)$, where

and

$$
s_{p} \in H^{0}\left(U, L^{\mu_{p}}\left(m_{p}+m_{p-1}\right) \otimes . \mathscr{H}\left(S_{p-1, p}\right)\right),
$$

$$
\begin{array}{rll}
s_{1}=f_{1} s_{2} & \text { on } & S_{1}, \\
s_{2}=f_{2} s_{3} & \text { on } & S_{2}, \\
\cdots & \ldots \ldots \ldots \ldots \ldots & \\
s_{N-1}=f_{N-1} s_{N} & \text { on } & S_{N-1} .
\end{array}
$$

Let $s$ be a local section of $E$ over $U$ and $t$ a local section of $E$ over $\tau(U)$. Then $t^{*}$ is a local section over $U$ and we can form the interesting expression

$$
\rho(s, t)=\sum_{p=1}^{N}(-1)^{p} e_{1} e_{2} \cdots e_{p-1} S_{p} t_{p}^{*} g_{1} \cdots g_{p-2} g_{p+1} \cdots g_{N-1} .
$$

By using the above formulae it is easy to check that this section of $0\left(2 m_{1}+\cdots+\right.$ $\left.2 m_{N-1}\right)$ vanishes on all of the spectral curves so we can define

$$
\langle s(\gamma), t(\gamma)\rangle=\frac{\rho(s, t)}{g_{1} \cdots g_{N-1}}(\gamma)
$$

Using the normalization for the $g_{p}$, the result on $*$ squared and the fact that $\sigma$ on functions is conjugation, it follows that the induced inner product on $H$ is 
hermitian. We shall show next that this inner product is compatible with the connection and Higgs field.

Consider any line $\gamma$ in $\mathbb{R}^{3}$ not on a spectral curve and the corresponding family $C_{x}, x \in \gamma$ of real sections in $T \mathbb{P}_{1}$. If $s$ and $t$ are sections of $E$ over any of these real sections, then the inner product $\langle s, t\rangle$ is a holomorphic function and therefore constant, so it can be determined by evaluating at any point, for example $\gamma$ or $\tau(\gamma)$ the two intersection points of the family of real sections. Choose two sections $s$ and $t$ of the bundle $H$ over the line in $\mathbb{R}^{3}$ so that as induced sections of $E$ over the family of real sections $s$ is constant at $\gamma$ and $t$ is constant at $\tau(\gamma)$. Then $\langle s, t\rangle$ evaluated at $\gamma$ is independent of where we are on the line in $\mathbb{R}^{3}$. Using the definition of the connection and Higgs field this means that if $\left(\nabla_{\gamma}-i \phi\right)(s)=0$ and $\left(\nabla_{\gamma}+i \phi\right)(t)=0$ then their inner product is constant. It is easy to deduce then that

$$
\frac{d}{d z}\langle s, t\rangle=\left\langle\left(\nabla_{z}-i \Phi\right) s, t\right\rangle+\left\langle s,\left(\nabla_{z}+i \Phi\right) t\right\rangle
$$

for $z$ a parameter along the line, and if we expand this out and equate the pieces which are symmetric and conjugate-symmetric in $s$ and $t$ we obtain the desired result. We have, in fact, only proved the result for lines not on the spectral curves, but these are dense in the space of all lines so the complete result follows by continuity.

Because the inner product is invariant under the connection, it is enough to show that it is definite somewhere to deduce that is definite everywhere or instead to show that it is asymptotically definite, which is the approach we shall take.

Consider again the family of real sections parametrised by the points of the line $\gamma=(0,0,-b / 2)$ in $\mathbb{R}^{3}$. Assume that we are in the generic situation of Sect. $\left.1 \mathrm{~d}\right)$; we have an asymptotic basis of sections ${ }^{i} s$ satisfying (1.22), (1.24), and so (1.25). It suffices to evaluate (1.26) at the point $(\eta, \zeta)=(0,0) \in C_{b}$ for $b \rightarrow \infty$, and show that it is positive. It is easy to check that this asymptotic basis is orthogonal; to check definiteness, we need to show that the sign of

$$
\frac{\left\langle{ }^{p} S,{ }^{p} S\right\rangle}{\left\langle{ }^{p+1} S,{ }^{p+1} S\right\rangle}(\gamma)
$$

is positive; at $(0,0)$, this equals

$$
-e_{p} \frac{{ }^{p} S_{p} \cdot{ }^{p} S_{p}^{*}}{{ }^{p+1} S_{p+1} \cdot{ }^{p+1} S_{p+1}^{*}-1}-\frac{g_{p+1}}{g_{p-1}}(\gamma) .
$$

Evaluating, we find

$$
\frac{-e_{p} \Pi\left(-\bar{\zeta}_{p-1, i}\right)^{-1} \cdot \Pi\left(-\bar{\zeta}_{p, m_{p}+i}\right)^{-1} g_{p+1}(0)}{\Pi\left(-\bar{\zeta}_{p, i}\right)^{-1} \Pi\left(-\bar{\zeta}_{p+1, m_{p+1}+i}\right)^{-1} g_{p-1}(0)} .
$$

Using $\zeta_{p, m_{p}+i}=-1 / \bar{\zeta}_{p, i}$, this is

$$
\frac{-e_{p}(-1)^{m_{p}+m_{p-1}} \Pi\left(\zeta_{p, i} \bar{\zeta}_{p, i}\right) \Pi\left(\bar{\zeta}_{p+1, i}\right) g_{p+1}(0)}{\Pi\left(\zeta_{p+1, i} \bar{\zeta}_{p+1, i}\right) \Pi\left(\bar{\zeta}_{p-1, i}\right) g_{p-1}(0)}
$$

from the fact that $g_{p}^{*}=(-1)^{m_{p}} g_{p}$, and that $b \zeta_{p, i} \rightarrow c_{p, i}$, one can easily show that 
$\left(\prod_{i=1}^{m_{p}} \bar{\zeta}_{p, i}\right) g_{p}(0)>0$ for all $p$. The expression above is then indeed positive, by the condition on the $e_{p}$.

To finish this section we show that when the spectral data is obtained from a monopole that the inner product on the monopole has this form and therefore the spectral data of a monopole satisfies the condition C-4. By continuity it suffices to work over a real section which doesn't intersect the intersections of the spectral curves.

Consider the part of the dual of figure (1.14) over a real section which is

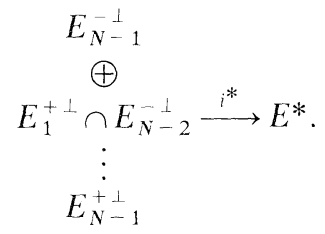

It is straightforward to check that the real structure on $E$ induces a map

$$
E / E_{p}^{+} \cap E_{N-p-1}^{-} \rightarrow E_{p}^{+\perp} \cap E_{N-p-1}^{-\perp}
$$

which covers the real structure on $T \mathbb{P}_{1}$ and is a multiple of $\sigma$ composed with multiplication by $g_{p-1} g_{p}$. It follows that the inner product on holomorphic sections of $E$ over a real section obtained by using the real structure on $E$ must take the form

$$
\langle s, t\rangle=\sum_{p=1}^{N} a_{p} \frac{s_{p} t_{p}^{*}}{g_{p-1} g_{p}} .
$$

We can now use the fact that this is a holomorphic function over the real section and defines a positive definite, hermitian form to reverse the arguments above and discover that if we set $a_{1}=-1$, then we have

and

$$
a_{p}=(-1)^{p} e_{1} \cdots e_{p-1}
$$

$$
-(-1)^{m_{p-1}+m_{p^{p}}} e_{p}>0 .
$$

To obtain the first condition one just applies the inner product to a section vanishing at a point of $S_{p} \cap C_{x}$, and to obtain the second one uses an asymptotic orthonormal basis as above.

\section{From Spectral Data to Nahm's Equations}

2a) Introduction and Notation. The purpose of this section is to obtain from the spectral data a solution $T_{i}(t)$ to Nahm's equations which satisfies the conditions $B$ of the introduction. Let us first combine the matrices $T_{i}(t)$ into a polynomial; define

$$
A(t, \zeta)=\left(T_{1}+i T_{2}\right)(t)-2 i T_{3}(t) \zeta+\left(T_{1}-i T_{2}\right)(t) \zeta^{2}=A_{0}(t)+A_{1}(t) \zeta+A_{2}(t) \zeta^{2}
$$

set $A_{\#}(t, \zeta)=\frac{1}{2} A_{1}(t)+A_{2}(t) \zeta$; Nahm's equations are then equivalent to

$$
\dot{A}+\left[A_{\#}, A\right]=0 \text {. }
$$


To solve this over each interval $\left(\mu_{p+1}, \mu_{p}\right)$, we first define a vector bundle $X$ over $\left(\mu_{p+1}, \mu_{p}\right)$; then we define a polynomial $\underline{A}(t, \zeta)$ with coefficients in $\Gamma(\operatorname{End}(X))$. From there, to get our matrices $A(t, \zeta)$, it suffices to choose a correct trivialisation of $X$; we will do this by specifying a connection. The main problem will be to show that the solution one obtains satisfies the boundary conditions B-2.

As all our computations will be on the fixed interval $\left(\mu_{p+1}, \mu_{p}\right)$, to reduce the number of indices we will define some notation particular to this section:

$t$ : flow parameter for solutions to Nahm's equations.

$z: \quad z=\mu_{p}-t$.

$m: \quad m=m_{p}$

$n: \quad n=m_{p-1}$.

$g: \quad g(\eta, \zeta)=\eta^{m}+a_{1}(\zeta) \eta^{m-1}+\cdots+a_{m}(\zeta)=g_{p}(\eta, \zeta) \cdot g=0$ is a local equation of $S_{p}$.

$h: \quad h(\eta, \zeta)=\eta^{n}+b_{1}(\zeta) \eta^{n-1}+\cdots b_{n}(\zeta)=g_{p-1}(\eta, \zeta) . h=0$ is a local equation for $S_{p-1}$.

$D: \quad D=S_{p-1, p} ; \tau(D)=S_{p, p-1}$.

M: $\quad M=\mathbb{C} \times S_{p}$; we will denote line bundles and divisors on $S_{p}$ and their lifts to $M$ by the same symbol.

$\mathscr{L}: \quad \mathscr{L}$ is the line bundle over $M$ defined by $\left.\mathscr{L}\right|_{\left\{=1 \times S_{p}\right.}=L^{z}$.

$X: \quad X=$ the direct image sheaf $P_{*}(\mathscr{L}(m+n-1)[-D]) \quad(P: M \rightarrow \mathbb{C}$ is the projection).

$\Gamma: \quad \Gamma=P_{*}(\mathscr{L}(m+n+1)[-D])$.

$\Xi: \quad \Xi=P_{*}(\mathscr{L}(m+n-1))$.

$N: \quad N=\mathbb{C} \times S_{p-1}$.

$Y$ : Defining $\mathscr{L}, P$ as above, but with respect to $N$ instead of $M, Y=$ $P_{*}(\mathscr{L}(m+n-1)[-D])$.

$g: \quad \tilde{g}(z, \eta, \zeta)=z^{m} g(\eta / z, \zeta)=\eta^{m}+z a_{1}(\zeta) \eta^{m-1}+\cdots+z^{m} a_{m}(\zeta)$.

$h: \quad \tilde{h}(z, \eta, \zeta)=z^{n} h(\eta / z, \zeta)$.

$z S_{p}: \quad z S_{p}$ is $S_{p}$ "shrunk" by a factor of $z$ in the $\eta$-direction; it is defined (fixing $z$ ) by $g(z, \eta, \zeta)=0$.

$F^{(k)}$ : The $k^{\text {th }}$ formal neighbourhood of the zero section in $T \mathbb{P}_{1}$, defined by $\eta^{k+1}=0$.

$\tilde{M}: \quad$ Surface in $\mathbb{C} \times T \mathbb{P}_{1}$ defined by $\tilde{g}(z, \eta, \zeta)=0$. Let $\tilde{P}: \tilde{M} \rightarrow C$ be the projection; then $\widetilde{P}^{-1}(0)=F^{(m-1)}$, and for $z \neq 0, \widetilde{P}^{-1}(z)=z S_{p}$. Again, bundles on $T \mathbb{P}_{1}$, their lifts to $\mathbb{C} \times T \mathbb{P}_{1}$, and their restriction to $\tilde{M}$ will be denoted by the same letter.

$\widetilde{\Xi}: \quad \widetilde{\Xi}=\widetilde{P}_{*}(L(m+n-1))$.

2b) Solution Over $\left(\boldsymbol{\mu}_{p+1}, \boldsymbol{\mu}_{p}\right)$. In this section, we will recall the version of the "Krichever construction" of the solution to Nahm's equations which is due to Hitchin [Hi2]. Let $V_{t}$ denote the sheaf $\left.L^{-t}(-1) \otimes\left(E / E_{p}^{+}+E_{N-p}^{-}\right)\right)$; for generic monopoles, $V_{t}=L^{\mu_{p}-t}(m+n-1)[-D]$. If $T_{a}$ denotes the fiber of $T \mathbb{P}_{1} \rightarrow \mathbb{P}_{1}$ at $a \in \mathbb{P}_{1}$, then one has the exact sequence over $S_{p}$ :

$$
\left.0 \rightarrow V_{t}(-1) \rightarrow V_{t} \rightarrow V_{t}\right|_{S_{p} \cap T_{a}} \rightarrow 0 .
$$

For $t \in\left(\mu_{p+1}, \mu_{p}\right), H^{0}\left(S_{p}, V_{t}(-1)\right)=0$, by the vanishing theorem (1.17). As the genus of $S_{p}$ is $(m-1)^{2}$, and the degree of $L^{\mu_{p}-t}(m+n-2)$ is $(m-1)^{2}-1$, Riemann Roch then implies that $H^{1}\left(S_{p}, V_{t}(-1)\right)=0$, and referring to (2.2), 


$$
\bar{X}_{t}=H^{0}\left(S_{p}, V_{t}\right) \simeq H^{0}\left(S_{p} \cap T_{a}, V_{t}\right) \simeq \mathbb{C}^{m} .
$$

That $h^{0}\left(S_{p} \cap T_{a}, V_{t}\right)=m$ is easy to see in the generic case, as $S_{p} \cap T_{a}$ consists of $m$ points, counted with multiplicity and $V_{t}$ is a line bundle. In general, if one writes out a local basis for $E, E_{p}^{+}, E_{n-p}^{-}$along $T_{a}$, it is straightforward to show that the number of independent sections of $V_{t}$ along $T_{a}$ is exactly the multiplicity of $S_{p}$. Next, as the dimension of $\bar{X}_{t}$ is constant in $t(2.3)$, the space $\bar{X}_{t}$ fit together nicely to form a vector bundle; in sheaf theoretic terms, $\bar{X}_{t}$ is the fiber $X_{t}$ at $t$ of the direct image sheaf $X$.

In a similar vein, one obtains

$$
\Gamma_{t} \simeq H^{0}\left(S_{p}, V_{t}(2)\right) \simeq \mathbb{C}^{3 m} .
$$

We next define the endomorphisms $A(t, \zeta)$. One way to do this $[\mathrm{AHH}]$ is to use (2.3). If $\zeta=\zeta_{0}$ corresponds to the point $a \in \mathbb{P}_{1}$, then one defines $\underline{A}\left(t, \zeta_{0}\right)$ by the commuting diagram:

$$
\begin{aligned}
& X_{t} \stackrel{\text { restr }}{\longrightarrow} H^{0}\left(S_{p} \cap T_{a}, V_{t}\right) \\
& \downarrow \downarrow \times \eta \\
& \downarrow \\
& X_{t} \stackrel{\text { 1estr }}{\longrightarrow} H^{0}\left(S_{p} \cap T_{a}, V_{t}\right)
\end{aligned} .
$$

A section at a point $\left(\zeta_{0}, \eta_{i 0}\right)$ of multiplicity $k$ of $S_{p} \cap T_{a}$ is a truncated power series $\Sigma \alpha_{j}\left(\eta-\eta_{i 0}\right)^{j}$; the map $\times \eta$ multiplies this series by $\eta$. Note that this means that the spectrum of $\underline{A}\left(t, \zeta_{0}\right)$ is the set of $\eta_{i 0}$; also, in the generic case when $V_{t}$ is a line bundle, that $\operatorname{dim} \operatorname{ker}(\eta\rceil-A(t, \zeta)) \leqq 1$, for all $\zeta, \eta$.

An equivalent way of defining $A\left(t, \zeta_{0}\right)$ is that of [Hi2]: the map

$$
H^{0}\left(S_{p}, \mathcal{O}(2)\right) \otimes X_{t} \rightarrow \Gamma_{t}
$$

has an $m$ dimensional kernel; taking a basis $\eta, 1, \zeta, \zeta^{2}$ of $H^{0}\left(S_{p}, \mathcal{O}(2)\right)$ (in the standard trivialisation), it is shown that there are endomorphisms $A_{0}, A_{1}, A_{2}$ of $X_{t}$, such that the map $s \mapsto \eta \otimes s-1 \otimes \underline{A}_{0}(s)-\zeta \otimes \underline{A}_{1}(s)-\zeta^{2} \otimes \underline{A}_{2}(s)$ is an isomorphism of $X_{t}$ onto the kernel of (2.5). One thus sees that $A(t, \zeta)$ is a polynomial of degree 2 in $\zeta$.

One must then give the trivialisation of $X$. As this is a bundle over a onedimensional base, then, up to an irrelevant overall change of basis this is equivalent to giving a connection on $X$. One fixes trivialisations of $L^{\mu}(k)$ over $T \mathbb{P}_{1}$ in which the transition functions from $U_{1}$ to $U_{0}$ are $\exp (\mu \eta / \zeta) \zeta^{k}$; this determines an isomorphism $e: H^{0}\left(S_{p} \cap U_{0}, V_{t}\right) \rightarrow H^{0}\left(S_{p} \cap U_{0}, V_{t_{0}}\right)$ for a fixed $t_{0}$. One then shows that there is a well defined connection $\nabla$ acting on sections of $X$ over $S_{p} \times \mathbb{C}$ defined by

$$
\nabla_{t}(s)=e^{-1}\left(\partial_{t} e+e \underline{A}_{\#}\right) s
$$

It then follows [Hi2] that writing $\underline{A}$ in a $\nabla$-flat basis gives a solution $A(t, \zeta)$ to Nahm's equation (2.1).

2c) Behaviour of $\boldsymbol{X}$ at $\boldsymbol{\mu}_{p}$. One thus obtains flows over the intervals $\left(\mu_{p+1}, \mu_{p}\right)$, $\left(\mu_{p}, \mu_{p-1}\right), \ldots$ etc. The problem then arises of studying boundary behaviour, at $\mu_{p}$, 
say. The particular boundary conditions we are studying are preserved under limits; therefore, it suffices to consider the generic case. As in the definition of the solution over $\left(\mu_{p+1}, \mu_{p}\right)$, we will analyse this in three steps, studying

i) the fiber $X_{\mu_{p}}$ of $X$ at $\mu_{p}$,

ii) the endomorphism $\underline{A}(t, \zeta)$ at $t=\mu_{p}$,

iii) the matrices $A(t, \zeta)$ at $t=\mu_{p}$.

The next three sections are devoted to this analysis. As everything we do concerns behaviour at $t=\mu_{p}$, it is more convenient to change our parameter, and set $z=\left(\mu_{p}-t\right)$. We incorporate this change into our definitions of $X$, $\underline{A}$, etc., and so we will refer to $X_{\mu_{p}}$ as $X_{0}, \underline{A}\left(\mu_{p}, \zeta\right)$ as $\underline{A}(0, \zeta)$, etc.

The first thing to do is to analyse $X$ at $z=0$. Approaching 0 from below, one is studying $L^{z}(m+n-1)[-D] \simeq L^{\mu_{p}-t}\left(m_{p}+m_{p-1}-1\right)\left[-S_{p-1}, p\right]$ over $S_{p}$ as $t \rightarrow \mu_{p}$; approaching $\mu_{p}$ from above, one is interested in $L^{\mu_{p-1}-t}\left(m_{p-1}+m_{p-2}-1\right)$ $\left[-S_{p-2, p-1}\right]$ over $S_{p-1}$ as $t \rightarrow \mu_{p}$; however, using the section of (1.8), this last bundle is isomorphic over $S_{p-1}$ to $L^{2}(m+n-1)[-D]$. Thus, in both cases one is looking at the same bundle, as $z \rightarrow 0$. Symmetry then allows us to consider only one case, say that of $S_{p}$.

By general theory, as $X$ is torsion free over a one dimensional base, $X$ is locally free. However, at $z=0$, at least for $m>n$, one does not necessary have the isomorphism (2.4). The evaluation map on the fiber $X_{0}$ of $X$ at $z=0$ :

$$
\mathrm{ev}_{0}: X_{0} \rightarrow H^{0}\left(S_{p}, \mathcal{O}(m+n-1)[-D]\right)
$$

is not necessarily surjective: the dimension of the space $H^{0}\left(S_{p}, L^{z}(m+n-1)[-D]\right)$ may jump (upward) at $z=0 . \mathrm{ev}_{0}$ is, however, injective; any section of $X$ mapping to zero in (2.7) corresponds to a local section of $\mathscr{L}(m+n-1)[-D]$ vanishing at $z=0$; this is then divisible by $z$, and so cannot be a generator of $X$. The problem is then to determine what the image is in (2.7).

To do this we will consider the sheaves $\Xi, \widetilde{\Xi}$ (see 2 .a) over $\mathbb{C}$, which in a similar fashion are locally free; the evaluation maps:

$$
\begin{aligned}
& \mathrm{ev}_{z}: \Xi_{z} \rightarrow H^{0}\left(S_{p}, L^{z}(m+n-1)\right), \\
& \tilde{\mathrm{ev}} \mathrm{v}_{z}: \widetilde{\Xi}_{z} \rightarrow H^{0}\left(\tilde{P}^{-1}(z), L(m+n-1)\right),
\end{aligned}
$$

are again injections, and are bijective for generic $z$. Sections of $X, \Xi$ (and of $\widetilde{\Xi}$ ) over $V \subset \mathbb{C}$ are to be thought of in terms of the corresponding sections of the bundles over $P^{-1}(V)\left(\tilde{P}^{-1}(V)\right)$; using the $U_{0}$ trivialisation, they will be thought of as functions $f(z, \eta, \zeta)$ over $P^{-1}(V)\left(\tilde{P}^{-1}(V)\right)$ satisfying certain constraints. $X$ is a subsheaf of $\Xi$, consisting of those sections which vanish along $\mathbb{C} \times D$.

There is a very useful link between $\Xi$ and $\widetilde{\Xi}$ :

Lemma (2.9). There is a map of sheaves

$$
\rho:\left.\left.L(k)\right|_{\tilde{M}} \rightarrow \mathscr{L}(k)\right|_{M}
$$

given locally as follows. If in the standard trivialisation over $U_{i}$ a section $s$ of $L(k)$ over $\tilde{M}$ is represented by $\tilde{f}_{i}(z, \eta, \zeta)$ then $\rho(s)$ is represented over $M$ by $f_{i}(z, \eta, \zeta)=$ $\tilde{f}_{i}(z, \eta z, \zeta)$. Away from $z=0$, this is an isomorphism. 
Proof. It suffices to verify that over $M, f_{0}=\exp (z \eta / \zeta) f_{1}$ : this is immediate.

Corollary (2.10). Taking direct images, there is a map of sheaves over $\mathbb{C}$

$$
\rho: \widetilde{\Xi} \rightarrow \Xi
$$

which is an isomorphism away from $z=0$.

We now study the sheaf $\tilde{\Xi}$; we find that the map ẽv of $(2.8)$ is always a bijection near $z=0$ by showing that the right-hand side has constant dimension. First, let us consider $z=0 ; \widetilde{P}^{-1}(0)$ is $F^{(m-1)}$. Note that $S L(2, \mathbb{C})$ acts on $F^{(m-1)}$; this action lifts to $L(k)[\mathrm{Hi} 2]$.

Lemma (2.11). One has the decomposition

$$
\begin{aligned}
H^{0}\left(F^{(m-1)}, L(m+n-1)\right) \simeq & H^{0}\left(F^{(m-1+n)}, L(m-1+n)\right) \\
& \oplus \eta H^{0}\left(F^{(m-1+n-2)}, L(m-1+n-2)\right) \\
\cdots \cdots & \\
& \oplus \eta^{\mathrm{s}} H^{0}\left(F^{(m-1+n-2 s)}, L(m-1+n-2 s)\right),
\end{aligned}
$$

where $s=\min (m-1, n)$. The $\eta^{i}$ are to be thought of as sections of $O(2 i)$, and there are implicit restrictions to $F^{(m-1)}$ on the right-hand side. The decomposition is $\operatorname{SL}(2, \mathbb{C})$ invariant.

Proof. We use Proposition 5.4 of [Hi2] repeatedly, which states:

Under restriction, one has an $S L(2, \mathbb{C})$ invariant isomorphism

$$
H^{0}\left(F^{(j)}, L(j)\right) \simeq H^{0}\left(F^{(0)}, L(j) \simeq H^{0}\left(\mathbb{P}_{1}, \mathcal{Q}(j)\right)\right.
$$

and

$$
H^{0}\left(F^{(j)}, L(j-1)\right)=0 .
$$

Let $\sigma$ be a section of $L(m+n-1)$ over $F^{(m-1)}$; then (2.12) implies that there is a section $\sigma_{0}$ of $L(m+n-1)$ over $F^{(m+n-1)}$ such that the restriction of $\sigma, \sigma_{0}$ to $F^{(0)}$ are the same: then $\sigma-\sigma_{0}=\eta \sigma_{1}$, say, over $F^{(m-1)}$, where as $\eta \sigma_{1}$ is defined over $F^{(m-1)}, \sigma_{1}$ is defined over $F^{(m-2)}$; as $\eta$ is a section of $\mathscr{O}(2), \sigma_{1}$ is a section of $L(m+n-1-2)$. One can then reapply $(2.12)$ to $\sigma_{1}$; iterating this procedure, one obtains

$$
\sigma-\left(\sigma_{0}+\eta \sigma_{1}+\cdots+\eta^{s} \sigma_{s}\right)=\eta^{s+1} \sigma_{s+1}
$$

where the $\sigma_{i}$ are restrictions to $F^{(m-1-i)}$ of sections of $L(m+n-1-2 i)$ over $F^{(m+n-1-2 i)}$, and $\sigma_{s+1} \in H^{0}\left(F^{(m-1-(s+1))}\right), L(m-1-2(s+1))$. This procedure truncates either when $s=m-1$ as then $\eta^{s+1}=0$, or when $s=n$, as then (2.13) implies that $\sigma_{s+1}=0$. It is easy to see that this decomposition is an isomorphism; $\operatorname{SL}(2, \mathbb{C})$ invariance follows from the naturality of the construction.

\section{Corollary (2.14).}

1) $h^{0}\left(F^{(m-1)}, L(m+n-1)\right)=m(n+1)$.

2) If $k>j$, the restriction map $H^{0}\left(F^{(k)}, L(k+j)\right) \rightarrow H^{0}\left(F^{(j)}, L(k+j)\right)$ is an isomorphism.

One next looks at the case $z \neq 0$ : 
Lemma (2.15) There is an interval I containing 0 such that for $z \neq 0, z \in I$,

$$
h^{0}\left(z S_{p}, L(m+n-1)\right)=m(n+1) .
$$

Proof. By Lemma (2.9), $\quad h^{0}\left(z S_{p}, L(m+n-1)\right) \simeq h^{0}\left(S_{p}, L^{z}(m+n-1)\right)$. By the vanishing theorem (1.17), and Riemann Roch, $h^{1}\left(S_{p}, L^{z}(m+n-2)[-D]\right)=0$; a fortiori, $h^{1}\left(S_{p}, L^{z}(m+n-1)\right)=0$. Riemann Roch then gives $h^{0}\left(S_{p}, L^{z}(m+n-1)\right)=$ $m(n+1)$.

As (2.8) is always an isomorphism, from (2.11) one therefore has a clear picture of what the fiber of $\widetilde{\Xi}$ is at $z=0$; the next step is to exploit this to study $\Xi$ at $z=0$, using the map $\rho$ of (2.9). We start with a lemma describing $H^{\circ}\left(S_{p}, \mathcal{O}(m+n-1)\right)$.

Lemma (2.16). The restriction map:

$$
H^{0}\left(T \mathbb{P}_{1}, \mathcal{O}(j)\right) \rightarrow H^{0}\left(S_{p}, \mathcal{O}(j)\right)
$$

is surjective. Its kernel is the set of sections of the form $g(\eta, \zeta) \cdot f(\eta, \zeta)$, with $f(\eta, \zeta) \in H^{O}\left(T \mathbb{P}_{1}, \mathcal{O}(j-2 m)\right)$, and so is zero for $j<2 m$.

Proof. We use the exact sequence $\left.0 \rightarrow \mathcal{O}(j-2 m) \rightarrow \mathcal{O}(j) \rightarrow \mathcal{O}(j)\right|_{S_{p}} \rightarrow 0$. The only nontrivial statement is surjectivity; to prove this, one shows that the map (multiplication by $g$ ) from $H^{1}\left(T \mathbb{P}_{1}, \mathcal{O}(j-2 m)\right) \rightarrow H^{1}\left(T \mathbb{P}_{1}, \mathcal{O}(j)\right)$ is injective. This is easy to see if one uses the explicit description of these spaces given in (1.2).

Combining (2.16) and (1.2), one sees that $H^{\mathrm{O}}\left(S_{p}, \mathcal{O}(m+n-1)\right)$ is composed of sections given in the $U_{0}$ trivialisation by $\sum_{i=0}^{m-1} \eta^{i} f_{i}(\zeta)$, where the $f_{i}$ are polynomials of degree $m+n-1-2 i\left(f_{l}=0\right.$ if $\left.m+n-1-2 i<0\right)$.

Proposition (2.17). The fiber $\Xi_{0}$ of $\Xi$ at $z=0$ is mapped under the evaluation map

$$
\mathrm{ev}_{0}: \Xi_{0} \rightarrow H^{\mathrm{O}}\left(S_{p}, \mathcal{O}(m+n-1)\right)
$$

to the subspace of sections of the form (in the $U_{0}$ trivialisation):

$$
\sum_{i=0}^{s} \eta^{i} f_{i}(\zeta), \text { where the } f_{i} \text { are polynomials of degree }(m+n-1-2 i),
$$

where $s=\min (m-1, n)$. Thus, $\mathrm{ev}_{0}$ is a bijection $\Leftrightarrow m-2 \leqq n$.

Proof. As the subspace of $H^{0}\left(S_{p}, \mathcal{O}(m+n-1)\right)$ represented by $(2.18)$ is in all cases of dimension $m(n+1)$, i.e. the dimension of $\Xi_{0}$, it suffices to show that all sections of the form (2.18) are in the image of $\mathrm{ev}_{0}$.

Let $\tilde{\xi}$ be a local section of $\tilde{\Xi}$ near $z=0 ; \tilde{\xi}$ can be written $\tilde{\xi}=\sum_{i=0}^{s} \eta_{i}^{i} \tilde{f}_{i}(\eta, \zeta)+O(z)$, where $\tilde{f}_{i}$ represents a section of $L(m+n-1-2 i)$ over $F^{(m-1-i)}$; then $\xi=\rho(\tilde{\xi})$ is $\sum_{i=0}^{s}(\eta z)^{i} \tilde{f}_{i}(\eta z, \zeta)+O(z)=\tilde{f}_{0}(0, \zeta)+O(z)$. As $\tilde{f}_{0}(0, \zeta)$ can be an arbitrary polynomial of degree $(m+n-1)$, this shows that the $i=0$ portion of $(2.18)$ is realised; to get the rest, one has to be a bit more careful.

Let $0 \leqq r \leqq s$. Suppose one has a section $\widetilde{\xi}=\Sigma \widetilde{\xi}_{i j k} \eta^{i \zeta^{j} z^{k}}$ of $\widetilde{\Xi}$, such that $\widetilde{\xi}_{i j k}=0$ for $i+k<r ; \xi=z^{-r} \rho(\tilde{\xi})$ is then a section of $\Xi$. We will show inductively in $r$ that 
one obtains in this way, at $z=0$, all sections in (2.18) of the form $\sum_{i=0}^{r} \eta^{i} f_{i}(\zeta)$.

Over $\tilde{M}$, one has the exact sequence of formal neighbourhoods of $\{z=0\}$,

$$
0 \rightarrow \mathcal{O}_{F^{(m-1)}} \rightarrow \mathcal{O}_{\left\{z^{k+1}=0\right\}} \rightarrow \mathcal{O}_{\left\{z^{k}=0\right\}} \rightarrow 0 .
$$

If one tensors this sequence with $L(m+n-1)$, and recalls from Hitchin ([Hi2, p. 174]) that $H^{1}\left(F^{(m-1)}, L(m+n-1)\right)=0$ for $n \geqq-1$, then it is clear that any section of $L(m+n-1)$ over $\left\{z^{k-1}=0\right\}$ extends to $\left\{z^{k}=0\right\}$. By Grothendieck's theorem (see, e.g. [Ha]), this means that any section over a formal neighbourhood of $z=0$ extends to an actual neighbourhood; it is thus sufficient to work over formal neighbourhoods.

Over $\{z=0\}=F^{(m-1)}$, consider a section $\tilde{\xi}$, given over $U_{0}$ by $\eta^{r} \tilde{f}_{0}(\eta, \zeta)$, and over $U_{1}$ by $\left(\eta / \zeta^{2}\right)^{r} \tilde{f}_{1}(\eta, \zeta)$, where the $\tilde{f}_{i}$ represent any element of $H^{0}\left(F^{(m-1+n-2 r)}\right.$, $L(m+1+n-2 r))$. One therefore has over $\tilde{M} \cap\{z=0\}$,

$$
\eta^{r} \tilde{f}_{0}(\eta, \zeta)=\exp (\eta / \zeta) \zeta^{m+n-1}\left(\left(\eta / \zeta^{2}\right)^{r} \tilde{f}_{1}(\eta, \zeta)\right)
$$

and so, over $T \mathbb{P}_{1}$,

$$
\eta^{r} \tilde{f}_{0}+\eta^{m} s=\exp (\eta / \zeta) \zeta^{m+n-1}\left(\left(\eta / \zeta^{2}\right)^{r} \tilde{f}_{1}\right)
$$

where $s$ is some function. However this implies that modulo $z^{r+1}$,

$$
\left.\left(\tilde{g} / \eta^{m-r}\right) \tilde{f}_{0}+\tilde{g} s=\exp (\eta / \zeta) \zeta^{m+n-1} \cdot\left(\tilde{g} / \eta^{m-r \zeta^{2 r}}\right) \cdot \tilde{f}_{1}\right)
$$

and so $\left(\tilde{g} / \eta^{m-r}\right) \tilde{f}_{0},\left(\tilde{g} / \eta^{m-r} \zeta^{2 r}\right) \tilde{f}_{1}$ define a section of $L^{m+n-1}$ over $\left\{z^{r+1}=0\right\}$; it is easy to check that this section satisfies $\xi_{i j k}=0$ for $i+k<r$, and that $\xi=z^{-r} \rho(\widetilde{\xi})$ is at $z=0$ of the form:

$$
\tilde{f}_{0}(0, \zeta)\left\{\eta^{r}+a_{1}(\xi) \eta^{r-1}+\cdots+a_{r}(\xi)\right\} .
$$

One sees from this that one can obtain all terms in (2.18) of order $r$ in $\eta$; inductively, one already had all terms of order $\leqq r-1$, and so one now has all terms of order $\leqq r$.

The last step is to reinsert the constraint of vanishing at $D$, that is to consider $X$ instead of $\Xi$. In this, it is useful to distinguish two cases:

i) $m \leqq n$.

In this case, the preceding discussion is not even necessary. One has, at $z=0$, the same vanishing theorem (1.17) one had for $z<0$; this implies $h^{0}\left(S_{p}, \mathcal{O}(m+n-1)\right.$ $[-D])=m$, which in turn gives us:

Proposition (2.20). If $m \leqq n$, the map $\mathrm{ev}_{0}$ in (2.7) is an isomorphism.

ii) $m>n$ :

Proposition (2.21). If $m>n$, $\mathrm{ev}_{0}\left(X_{0}\right)$ in (2.7) splits into two summands $Z_{0}^{\prime} \oplus Z_{0}^{\prime \prime}$.

$Z_{0}^{\prime}$ is of dimension $(m-n)$ and consists of sections vanishing on $S_{p} \cap S_{p-1}$; in the $U_{0}$ trivialisation, these are of the form $h(\eta, \zeta) \cdot p(\zeta), p(\zeta)$ a polynomial of degree $\leqq m-n-1$.

$Z_{0}^{\prime \prime}$ is of dimension $n$, and consists of all the sections of the form $\sum_{s=0}^{n-1} \eta^{i} t_{i}(\zeta)$ which vanish on $D$. 
Proof. Let $W=H^{0}\left(S_{p}, \mathcal{O}(m+n-1)[-D]\right)$; we will show that $\operatorname{ev}_{0}\left(X_{0}\right)=W \cap$ $\mathrm{ev}_{0}\left(\Xi_{0}\right)$; as $\operatorname{ev}_{0}\left(X_{0}\right) \subset W \cap \operatorname{ev}_{0}\left(\Xi_{0}\right)$, it suffices then to show that $W \cap \operatorname{ev}_{0}\left(\Xi_{0}\right)$ is of dimension $m$.

By Proposition (2.17), the space $Z_{0}^{\prime}$ sits inside $W \cap \mathrm{ev}_{0}\left(\Xi_{0}\right)$, and has dimension $(m-n)$; furthermore, if $s$ is any element of $W \cap \mathrm{ev}_{0}\left(\Xi_{0}\right)$ then there exists an element $s^{\prime}$ of $Z_{0}^{\prime}$ (determined by the $\eta^{n}$ component of $s$ ) such that $s-s^{\prime}=s^{\prime \prime} \in Z_{0}^{\prime \prime}$. The only thing to be shown is that $Z_{0}^{\prime \prime}$ has dimension $n$. As $\operatorname{ev}_{0}\left(X_{0}\right) \subset Z_{0}^{\prime} \oplus Z_{0}^{\prime \prime}$, its dimension is at least $n$. Let $s^{\prime \prime} \in Z_{0}^{\prime \prime}$. By (2.16), it is the restriction to $S_{p}$ of a section $t^{\prime \prime}$ over $T \mathbb{P}_{1}$ which vanishes on $D$; as $t^{\prime \prime}$ is of the form $\sum_{i=0}^{n-1} \eta^{i} p_{i}(\zeta), t^{\prime \prime}$ does not vanish when restricted to $S_{p-1}$. One then has an injective map from $Z_{0}^{\prime \prime}$ to $H^{0}\left(S_{p-1}, \mathcal{O}(m+n-1)[-D]\right)$; by case i), this latter space is of dimension $n$, and so $\operatorname{dim}\left(Z_{0}^{\prime \prime}\right)=n$.

The proof of (2.21) also tells us how the bundles $X, Y$ should be glued at $z=0$. One uses the diagram:

$$
H^{\mathrm{O}}\left(S_{p}, \mathcal{O}(m+n-1)[-D]\right) \quad H^{0}\left(T \mathbb{P}_{1}, \mathcal{O}(m+n-1) \otimes \mathscr{I}(D)\right)
$$

When $m>n, Y_{0}$ is mapped isomorphically into the summand $Z_{0}^{\prime \prime}$ of $X_{0}$. Note that when $m=n$, the maps in (2.22) are both isomorphisms.

2d) The Endomorphism $\underline{A}(z, \zeta)$ at $\boldsymbol{\mu}_{p}$. Having now established what the fiber of $X$ is at $z=0$, we turn our attention to the behaviour of the endomorphisms $A(z, \zeta)$ defined by $(2.4)$ as $z \rightarrow 0$. As $z \rightarrow 0$ from above, $\underline{A}(z, \zeta)$ is defined using the curve $S_{p-1}$; as $z \rightarrow 0$ from below, $\underline{A}(z, \zeta)$ is defined using the curve $S_{p}$. As the two situations are symmetric, we confine our study to the latter. As before, it will be useful to distinguish the cases: $m<n, m>n, m=n$.

i) $m<n$.

Referring to the exact sequence (2.2), and case b) of the vanishing theorem, (1.17), one can apply the same arguments one had for $t \in\left(\mu_{p+1}, \mu_{p}\right)$ to the case $t=\mu_{p}$ $(z=0)$, showing that the restriction map in (2.3) is an isomorphism at $z=0$. This enables one to show that there is a well defined finite limit $\underline{A}(0, \zeta)$. of $\underline{A}(z, \zeta)$.

ii) $m>n$.

In this case, the restriction map in (2.3) is no longer an isomorphism; as we shall see, $\underline{A}(z, \zeta)$ has a pole at $z=0$. As before, when analysing this case, it is more convenient to work with $\tilde{M}$ than with $M$. Let

$$
\mathscr{I}_{p}=\text { ideal of functions } \Sigma f_{i j k} \eta^{i} \zeta^{j} z^{k} \text { with } f_{i j k}=0 \text { for } i+k<p \text {. }
$$

The proof of (2.17) gave an isomorphism

$$
z^{-n} \rho: \mathscr{I}_{n} \widetilde{\Xi} \rightarrow \Xi \text {. }
$$

Let $\tilde{X}=\left(z^{-n} \rho\right)^{-1}(X)$. In a similar fashion, define $\tilde{\Gamma}=\left(z^{-n} \rho\right)^{-1}(\Gamma)$. The following lemma explains how the maps $\eta, \underline{A}(z, \zeta)$ involved in (2.4) transport from $M$ to $\tilde{M}$ : 
Lemma (2.24). The diagram

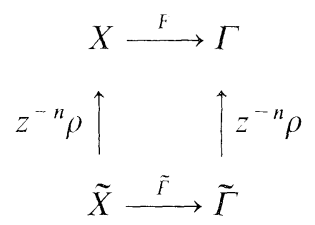

commutes, where either i) $F=z \eta, \tilde{F}=\eta$

ii) $F=A(z, \zeta)=\tilde{F}$.

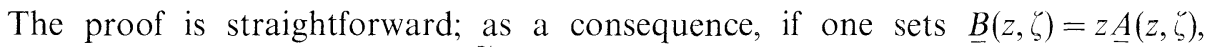
$(\eta-\underline{A}(z, \zeta)) X=0 \Leftrightarrow(\eta-\underline{B}(z, \zeta)) \tilde{X}=0$. We will compute $\underline{B}$ instead of $\underline{A}$.

As a first step, we define near $z=0$ two subbundles $Z^{\prime}, Z^{\prime \prime}\left(\zeta_{0}\right)$ of $X$ of rank $(m-n), n$ respectively, which extend the summands $Z_{0}^{\prime}, Z_{0}^{\prime \prime}$ of $X_{0}$ defined in (2.21), in such a way that:

- local sections of $Z^{\prime}$ correspond over $M$ to sections which are divisible by $h$, to order $(m-n)$ (inclusively) in $z$.

- local sections of $Z^{\prime \prime}\left(\zeta_{0}\right)$, considered as sections over $M$, restricted to $\zeta=\zeta_{0}$ and written, using Lagrange interpolation in $\eta$, as polynomials in $\eta$ of degree $<m$, are of the form $\sum_{i=0}^{n-1} a_{i}(z) \eta^{i}$; all such functions can be obtained.

The subbundle $Z^{\prime}$ is obtained by constructing the appropriate subbundle $\tilde{Z}^{\prime}$ of $\tilde{X}$. Apply the division algorithm in $\eta$ to obtain:

$$
g(\eta, \zeta)=k(\eta, \zeta) h(\eta, \zeta)+r(\eta, \zeta)
$$

where $k$ is of degree $(m-n)$ in $\eta, r$ of degree less than $n$ in $\eta$. One has

$$
z^{m} g(\eta / z ; \zeta)=\left(z^{m-n} k(\eta / z, \zeta)\right)\left(z^{n} h(\eta / z, \zeta)\right)+z^{m} r(\eta / z, \zeta)
$$

rewrite this as

$$
\tilde{g}(z, \eta, \zeta)=\tilde{k}(z, \eta, \zeta) \cdot \tilde{h}(z, \eta, \zeta)+z^{m} r(\eta / z, \zeta) .
$$

Thus, modulo the ideal $\mathscr{I}_{m}(2.23), \tilde{g}=\tilde{k} \tilde{h}$. Now consider the surface $\tilde{Q} \subset \mathbb{C} \times T \mathbb{P}_{1}$ defined by $\tilde{k}=0$; let $\tilde{R}: \tilde{Q} \rightarrow \mathbb{C}$ be the projection. Define

$$
\tilde{\Sigma}=\tilde{R}_{*}(L(m-n-1))
$$

then $\tilde{\Sigma}_{0}$, by $(2.12)$, is

$$
H^{0}\left(F^{(m-n-1)}, L(m-n-1)\right) \simeq H^{0}\left(F^{(0)}, L(m-n-1)\right) .
$$

Let $\widetilde{C}_{i}, i=1, \ldots, m-n$ be the elements of a local basis of $\tilde{\Sigma}$, which over $F^{(0)}$ correspond to the section $\zeta^{i-1} ; \widetilde{C}_{i}$ can be represented by functions $\tilde{f}_{j i}$ over $U_{j}$ with $\tilde{f}_{0 i}=\exp (\eta / \zeta) \zeta^{m-n-1} \tilde{f}_{1 i}+\tilde{k} s_{i}$, where $s_{i}$ is some function. Therefore,

$$
\tilde{h} \tilde{f}_{0 i}=\exp (\eta / \zeta) \zeta^{m+n-1}\left(\left(\tilde{h} / \zeta^{2 n}\right) \tilde{f}_{1 i}\right)+\tilde{k} \tilde{h} s
$$

i.e., modulo $\mathscr{I}_{m}, \tilde{h} \tilde{f}_{0 i}=\exp (\eta / \zeta) \zeta^{m+n-1}\left(\left(\tilde{h} / \zeta^{2 n}\right) \tilde{f}_{1 i}\right)+\tilde{g} s$; modulo $\mathscr{I}_{m}$ one has local sections of $L(m+n-1)$ over $\tilde{M}$ which are divisible by $\tilde{h}$. One checks, order by 
order in $z$, similarily to the proof of Lemma (2.17), that such sections extend to a neighbourhood of $z=0$. Applying $z^{-n} \rho$ to the $\tilde{e}_{i}$, one obtains sections $e_{1} \cdots e_{m-n}$, divisible by $h$ to order $(m-n)$ in $z ; Z^{\prime}$ is the subbundle spanned by these sections.

The definition of $Z^{\prime \prime}\left(\zeta_{0}\right)$ is more straightforward. The elements of $X_{z}$ whose Lagrange interpolations over $\zeta=\zeta_{0}$ are of the form $\sum_{i=0}^{n-1} a_{i} \eta^{i}$, form a rank $n$ subbundle of $X_{z}$; at $z=0$, this is a consequence of (2.17), and away from $z=0$, of the isomophism (2.3). One has a natural basis $e_{n-m+1} \cdots \mathrm{e}_{m}$ of $Z^{\prime \prime}\left(\zeta_{0}\right)$ restricting over $\zeta=\zeta_{0}$ to $\eta^{n-1}, \ldots, 1$.

One would like to study $\underline{B}\left(z, \zeta_{0}\right)$ at $z=0$; as for $\underline{A}$, one has two definitions of $\underline{B}$. The first uses a diagram similar to (2.4).

$$
\begin{aligned}
& \tilde{X}_{z} \stackrel{\text { restr }_{z, a}}{\longrightarrow} H^{0}\left(z S_{p} \cap T_{a}, L(m+n-1)\right) \simeq \mathbb{C}^{m} \\
& \underline{B}(z, \zeta) \downarrow\lfloor\times \eta \\
& \tilde{x}_{z} \stackrel{\text { testt }_{z, a}}{\longrightarrow} H^{\mathrm{O}}\left(z S_{p} \cap T_{a}, L(m+n-1)\right) \simeq \mathbb{C}^{m}
\end{aligned}
$$

Again, the spaces on the right-hand side are to be thought of as the space $V$ of polynomials in $\eta$ of degree less than $m$, using Lagrange interpolation. The diagram (2.30) defines $\underline{B}(z, \zeta)$, as long as restr ${ }_{z, a}$ is an isomorphism; the problem is that this is not the case at $z=0$. Let us consider the action of $\operatorname{restr}_{z, a}$ on the subbundles $\tilde{Z}^{\prime}, \tilde{Z}^{\prime \prime}\left(\zeta_{0}\right)$ of $\tilde{X}$ corresponding under $z^{-n} \rho$ to $Z^{\prime}, Z^{\prime \prime}\left(\zeta_{0}\right)$. Remember $e_{i}=z^{-n} \rho\left(\tilde{e}_{i}\right)$. For $e_{i}, i=1, \ldots, m-n$ (i.e. the local basis of $\left.\tilde{Z}^{\prime}\right) \operatorname{restr}_{0, a}\left(\tilde{e}_{i}\right)=\eta^{n}$ (polynomial $p_{i}(\eta)$ ), as $\tilde{h}=\eta^{n}$ over $z=0$. Referring to (2.11), (2.28), the $\tilde{e}_{i}$ at zero form a basis of $\eta^{n} H^{0}\left(F^{(m-n-1)}, L(m-n-1)\right)$; as $H^{0}\left(F^{(m-n-1)}, L(m-n-2)\right)=0$, the $\operatorname{restr}_{0, a}\left(\tilde{e}_{i}\right)$ form a basis of the subspace $V_{n}$ of $V$ consisting of polynomials of the form $\sum_{i=n}^{m} a_{i} \eta^{i}$.

On the other hand, for $i=m-n+1, \ldots, m$, $\operatorname{restr}_{z, a}\left(\tilde{e}_{i}\right)$ has leading term $\eta^{m-i} z^{i-n-m}$, and so $\operatorname{restr}_{0, a}\left(\tilde{e}_{i}\right)=0 ; Z^{\prime \prime}\left(\zeta_{0}\right)$ maps to zero.

We modify the map restr ${ }_{a}$ to obtain an isomorphism at $z=0$. Set for $z=0$,

$$
\begin{aligned}
m \operatorname{restr}_{z, a}\left(\tilde{e}_{i}\right) & =\operatorname{restr}_{z, a}\left(\tilde{e}_{i}\right), \quad i=1, \ldots, m-n, \\
& =z^{m-n-1} \operatorname{restr}_{z, a}\left(\tilde{e}_{i}\right) \quad i=m-n+1, \ldots, m .
\end{aligned}
$$

Then $m$ restr $_{z, a}$ is an isomorphism for all $z \neq 0$, and the limit $m$ restr $_{0, z}$ is an isomorphism also. Define $\underline{B}^{\prime}\left(z, \zeta_{0}\right)$ by

$$
\begin{gathered}
\tilde{X}_{z} \stackrel{m \text { restr }_{z, a}}{\longrightarrow} V \\
\underline{B}^{\prime}\left(z, \zeta_{0}\right) \downarrow \\
\tilde{X}_{z} \stackrel{m \text { restr }_{z, a}}{\longrightarrow} V
\end{gathered}
$$

$\underline{B}^{\prime}$ is continuous, well defined at $z=0$; in fact, changing the basis $\tilde{e}_{1} \cdots \tilde{e}_{m-n}$ of $\tilde{Z}^{\prime}$ so that $\operatorname{restr}_{0 . a}\left(\tilde{e}_{i}\right)=\eta^{m-i+1}$, 


$$
\begin{aligned}
& \underline{B}^{\prime}\left(0, \zeta_{0}\right)\left(e_{i}\right)=0 \quad \text { if } \quad i=1 \\
& =e_{i-1} \quad \text { if } \quad i=2, \ldots, m .
\end{aligned}
$$

\section{Proposition (2.32).}

i) $\underline{B}(z, \zeta)$ is continuous at $z=0$.

ii) With respect to the decomposition $\tilde{X}=\tilde{Z}^{\prime} \oplus Z^{\prime \prime}(\zeta), \underline{B}(z, \zeta)$ is of the form:

$$
\left[\begin{array}{cc}
\alpha(z, \zeta) & O(z) \\
O\left(z^{m-n}\right), & O(z)
\end{array}\right] .
$$

iii) Writing $\alpha(z, \zeta)=\alpha_{0}(z)+\alpha_{1}(z) \zeta+\alpha_{2}(z) \zeta^{2}$ the $\alpha_{i}(0)=z_{i}, i=0,1,2$ are the generators of an irreducible representation of $\operatorname{sl}(2, \mathbb{C})$.

Proof. i) One uses the expression above for $\underline{B}^{\prime}(z, \zeta)\left(e_{i}\right)$; one gets, e.g., for $\zeta=\zeta_{0}$ :

$$
\begin{aligned}
\underline{B}\left(z, \zeta_{0}\right)\left(e_{i}\right)=O(z) & i=1, \\
e_{i-1}+O(z), & i=2, \ldots, m-n, \\
z e_{i-1}, & i=m-n+1, \ldots, m .
\end{aligned}
$$

iii) Equation (2.33) shows that $\underline{B}(0, \zeta)\left(Z_{0}^{\prime}\right) \subset Z_{0}^{\prime}$; to see that one gets the representation, it is more convenient to use the second definition of $\underline{B}$ analogous to that of $\underline{A}$ given after (2.5): one has the map

$$
H^{0}\left(T \mathbb{P}_{1}, \mathcal{O}(2)\right) \otimes \tilde{X} \rightarrow \tilde{\Gamma} .
$$

$\underline{B}$ is defined by $(\eta-\underline{B}(z, \zeta))(\tilde{X})=0$. At $z=0$, restricted to $Z_{0}^{\prime}$, the $\operatorname{map} \times \eta$ is:

$$
\times \eta: \eta^{n} H^{0}\left(F^{(m-n-1)}, L(m-n-1)\right) \rightarrow \eta^{n} H^{0}\left(F^{(m-n-1)}, L(m-n+1)\right) .
$$

Using (2.11), this becomes:

$$
\times \eta: \eta^{n} H^{0}\left(F^{(m-n-1)}, L(m-n-1)\right) \rightarrow \eta^{n}\left[\begin{array}{r}
H^{0}\left(F^{(m-n+1)}, L(m-n+1)\right) \\
\oplus \eta H^{0}\left(F^{(m-n-1)}, L(m-n-1)\right) \\
\oplus \eta^{2} H^{0}\left(F^{(m-n-3)}, L(m-n-3)\right)
\end{array}\right]
$$

$\times \eta$ is just the $\operatorname{Sl}(2, \mathbb{C})$ invariant isomorphism into the second summand. Referring to Hitchin [Hi2, p. 178], if one sets in the basis $\tilde{e}_{i}$ which, at $z=0$, over $\eta^{n+1}=0$, corresponds to the monomial $\eta^{n \varphi^{i-1}}$ :

$$
\begin{aligned}
& a_{0}\left(\tilde{e}_{j}\right)=(j-(m-n)) \tilde{e}_{j+1}, \\
& a_{1}\left(\tilde{e}_{j}\right)=(-2 j+(m-n)+1) \tilde{e}_{j}, \\
& a_{2}\left(\tilde{e}_{j}\right)=(j-1) \tilde{e}_{j-1},
\end{aligned}
$$

then the $a$ 's give an irreducible representation of $\operatorname{sl}(2, \mathbb{C})$, and $\left(\eta+\Sigma a_{i} \zeta^{i}\right)\left(\tilde{Z}_{0}^{\prime}\right)=0$. ii) One must show that the fact that $\underline{B}(z, \zeta)\left(\tilde{Z}_{0}^{\prime}\right) \subset \tilde{Z}_{0}^{\prime}$ at $z=0$ extends to order $(m-n)$ in $z$. For this, we go back to the construction of the subbundle $\tilde{Z}^{\prime}$; referring to (2.25), (2.27) and setting $S^{\prime}$ to be the curve defined by $(k=0)$, then $\tilde{R}^{-1}(z)=z S^{\prime}$, for $z \neq 0$, and $\widetilde{R}^{-1}(0)=F^{(m-n-1)}$. One can show that $h^{0}\left(\tilde{R}^{-1}(z)\right.$, $L(m-n+1))=(m-n)$ for $z$ near 0 , and the restriction maps: 


$$
\tilde{\Sigma}_{z}=H^{0}\left(\tilde{R}^{-1}(z), L(m-n-1)\right) \rightarrow H^{0}\left(\tilde{R}^{-1}(z) \cap T_{a}, L(m-n-1)\right) \simeq \mathbb{C}^{m-n}
$$

are isomorphisms for all $z$ near 0 . This implies that, as before, there are $\hat{\alpha}_{i}(z)$ with $\left(\eta-\hat{\alpha}_{0}(z)-\hat{\alpha}_{1}(z) \zeta-\hat{\alpha}_{2}(z) \zeta^{2}\right) \tilde{\Sigma}=0$. then

Again, let a local basis of sections of $\tilde{\Sigma}$ be represented by functions $\tilde{f}_{j i}$ on $U_{j}$;

$$
\left(\tilde{f}_{01}, \ldots, \tilde{f}_{0, m-n}\right)\left(\eta-\hat{\alpha}_{0}(z)-\hat{\alpha}_{1}(z) \zeta-\hat{\alpha}_{2}(z) \zeta^{2}\right)=\left(\tilde{v}_{1}, \ldots, \tilde{v}_{m-n}\right) \tilde{k}
$$

where the $\tilde{v}_{i}$ are functions; multiply this by $\tilde{h}$ :

$$
\left(\tilde{h} \tilde{f}_{01}, \ldots, \tilde{h} \tilde{f}_{0, m-n}\right)\left(\eta-\hat{\alpha}_{0}(z)-\hat{\alpha}_{1}(z) \zeta-\hat{\alpha}_{2}(z) \zeta^{2}\right)=\left(\tilde{v}_{1}, \ldots, \tilde{v}_{m-n}\right) \tilde{g}
$$

to order $(m-n)$ in $z$. Note that the $\tilde{h} \tilde{f}_{o i}$ are precisely what defined the basis of sections $\tilde{e}_{i}$ of $\tilde{Z}^{\prime}$. One has then has, to order $(m-n)$ in $z$, that $\hat{\alpha}_{i}(z)=\alpha_{i}(z)$, and that $\left(\eta-\Sigma \alpha_{i}(z) \zeta^{i}\right) \tilde{Z}^{\prime}=0$.

This finishes the proof that the endomorphisms $\underline{A}$ have the correct boundary behaviour on both sides of $\mu_{p}$ for $m \neq n$; one then must check that they patch together. Referring to (2.24), we have that in a basis of $X$ whose first $(m-n)$ elements generate $Z^{\prime}$ and whose last $n$ elements at $z=0$ generate $Z_{0}^{\prime \prime}, \underline{A}(z, \zeta)$ is of the form, near $z=0$ :

$$
\left[\begin{array}{cc}
a(\zeta) / z+a^{\prime}(\zeta)+O(z), & b(\zeta)+O(z) \\
O\left(z^{m-n-1}\right), & c(\zeta)+O(z)
\end{array}\right]
$$

with $a(z)$ corresponding to an irreducible representation of $S U(2)$. This is computed using $S_{p}$, for $z \in\left(0, \mu_{p}-\mu_{p+1}\right)$; similarily, one has, for $z \in\left(\mu_{p}-\mu_{p-1}, 0\right)$, a solution $\underline{A}(z, \zeta)$, computed using $S_{p-1} ; \underline{A}(z, \zeta)$ is there an endomorphism of the rank $\bar{n}$ bundle $Y$, and we saw that there is a well defined limit $\underline{A}^{-}(\zeta)$ at $z=0$. In fact, under the identification of $Y_{0}$ with $Z_{0}^{\prime \prime}$, we have that $\underline{A}^{-}=c$, as follows.

Remember from (2.16), that, for $m>n, \quad H^{0}\left(S_{p}, \mathcal{O}(m+n+1)[-D]\right) \simeq$ $H^{0}\left(T \mathbb{P}_{1}, \mathcal{O}(m+n+1) \otimes \mathscr{I}(D)\right)$. Thus, at $z=0$, if $\left(e_{1} \cdots e_{m}\right)(\eta-\underline{A}(z, \zeta))=0$ over $S_{p}$, the same is true over $T \mathbb{P}_{1}$; in particular, at $z=0$,

$$
0=\eta e_{m-n+i}+\sum_{j=1}^{m-n} e_{j} b_{j, i}(\zeta)+\sum_{j=1}^{n} e_{m-n+j} c_{j, i}(\zeta)
$$

over $T \mathbb{P}_{1}$. However, at $z=0$, over $S_{p-1}, e_{1}, \ldots, e_{m-n}$ vanish, and so, over $S_{p-1}$,

$$
0=\eta e_{n-m+i}+\sum_{j=1}^{n} e_{m-n+j} c_{j i}(\zeta)
$$

Under the identification (2.22) this forces $c(\zeta)=\underline{A}^{-}(\zeta)$.

iii) The Case $m=n$.

This case is rather similar to that of $m<n$; again, the vanishing theorem (1.17) applies in the limit at $z=0$ and so one has well defined, finite limits $\underline{A}^{-}(\zeta), \underline{A}^{+}(\zeta)$, as $z$ tends to zero from below or above.

$\underline{A}^{+}(\zeta)$ and $\underline{A}^{-}(\zeta)$, are not the same, however. Whereas $X_{0}$ and $Y_{0}$ are well identified by $(2.22)$, the spaces $\Gamma_{0}$ and $\Delta_{0}=H^{0}\left(S_{p-1}, \mathcal{O}(m+n+1)[-D]\right)$ are not, via the analogous diagram: 


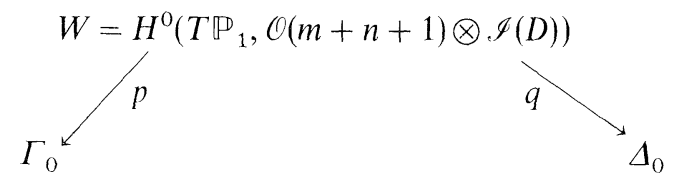

The map $p$ has a two dimensional kernel, consisting of sections of the form $(x+y \zeta) g, x, y \in \mathbb{C}$; similarly, the map $q$ has a kernel of sections of the form $(x+y \zeta) h$. Referring to (2.5), this allows $\underline{A}^{+}$and $\underline{A}^{-}$to be different.

Represent a basis of sections of $X_{0} \cong Y_{0} \cong \bar{H}^{0}\left(T \mathbb{P}_{1}, \mathcal{O}(m+n-1) \otimes \mathscr{I}(D)\right)$ by polynomials $v_{i}$ in $\zeta, \eta$ of degree less than $m$ in $\eta ; A^{+}, A^{-}$are defined by

$$
\begin{gathered}
\left(v_{1}, \ldots, v_{m}\right)\left(\eta-\underline{A}^{-}(\zeta)\right)=\left(s_{0 i}+\zeta s_{11}, \ldots, s_{0 m}+\zeta s_{1 m}\right) g, \\
\left(v_{1}, \ldots, v_{m}\right)\left(\eta-\underline{A}^{+}(\zeta)\right)=\left(t_{0 i}+\zeta t_{11}, \ldots, t_{0 m}+\zeta t_{1 m}\right) h,
\end{gathered}
$$

$s_{j i}, t_{j i} \in \mathbb{C} .\left(s_{0 i}+s_{1 i} \zeta\right)$ is the $\left(\eta^{m-1}\right)$ term of $v_{i}$, as is $\left(t_{0 i}+t_{1 i} \zeta\right)$; therefore $s_{0 i}=t_{0 i}$, $s_{1 i}=t_{1 i}$. Writing $s_{j}=\left(s_{j 1}, \ldots, s_{j m}\right)$, we get

$$
\left(v_{1} \cdots v_{m}\right)\left(\underline{A}^{+}(\zeta)-\underline{A}^{-}(\zeta)\right)=(h-g)\left(s_{0}+\zeta s_{1}\right) .
$$

$(h-g)$ represents a section of $\mathcal{O}(2 m)$, which vanishes on $S_{p} \cap S_{p-1}$; as $H^{0}\left(S_{p}, \mathcal{O}(2 m)[-D]\right) \cong H^{0}\left(S_{p}, \mathcal{O}(2 m-1)[-D]\right) \otimes H^{0}\left(S_{p}, \mathcal{O}(1)\right),(h-g)$ decomposes as $\sum_{i=1}^{m} v_{i}\left(u_{0 i}+\zeta u_{1 i}\right)$, with $u_{j i} \in \mathbb{C}$. Writing $u_{j}=\left(u_{j 1}, \ldots, u_{j m}\right)$, and substituting into (2.36) yields

and so:

$$
\left(v_{1}, \ldots, v_{m}\right)\left(\underline{A}^{+}(\zeta)-\underline{A}^{-}(\zeta)\right)=\left(v_{1}, \ldots, v_{m}\right)\left(u_{0}+\zeta u_{1}\right)^{T}\left(s_{0}+\zeta s_{1}\right),
$$

\section{Proposition (2.38).}

$$
\underline{A}^{+}(\zeta)-\underline{A}^{-}(\zeta)=\left(u_{0}+\zeta u_{1}\right)^{T}\left(s_{0}+\zeta s_{1}\right),
$$

i.e., $\underline{A}^{+}(\zeta)-\underline{A}^{-}(\zeta)$ is of rank one.

2e) The Matrices $A(z, \zeta)$ at $\boldsymbol{z}=\mathbf{0}$. One remembers that before one could obtain a solution to Nahm's equations, one had to trivialise the bundle $X$ so that the endomorphisms $A(z, \zeta)$ could be written as matrices $A(z, \zeta)$; this trivialisation is chosen to be flat with respect to a certain connection $\nabla$.

The definition (2.6) of the connection involved the maps $A(z, \zeta)$. When these have finite, well defined limits at $z=0$, there is no problem obtaining a smooth $\nabla$-flat trivialisation at $z=0$; the boundary behaviour of $A$ is then, in essence, that of $A$, that is:

-for $m<n$, there is a finite well defined limit $A(0, \zeta)=\lim _{z \rightarrow 0^{+}} A(z, \zeta)$.

-for $m=n$, there are well defined limits $A^{-}(\zeta), A^{+}(\zeta)$ with

$$
A^{+}(\zeta)-A^{-}(\zeta)=\left(\mu_{0}+\mu_{1} \zeta\right)^{T}\left(a_{0}+a_{1} \zeta\right) \text {. }
$$

It is the case $m>n$ which poses the problem, as $A(z, \zeta)$ has a pole at $z=0$. Let $e_{1}, \ldots, e_{m}$ be a local basis of $X$; set $C(z, \zeta)=C_{0}(z)+C_{1}(z) \zeta+C_{2}(z) \zeta^{2}$ to be the expression of $A(z, \zeta)$ in this trivialisation of $X$. If $s$ is a section of $X$, let $(s)_{0}$ 
denote the function representing $s$ in the $U_{0}$ trivialisation. The connection is defined by:

$$
\left(\nabla_{z} e_{i}\right)_{0}=\frac{\partial\left(e_{i}\right)_{0}}{\partial z}+\sum_{j}\left(e_{j}\left(\frac{C_{1}}{2}+\zeta C_{2}\right)_{j i}\right)_{0} .
$$

Note that this implies that there is a matrix

$$
D_{j i}(z, \zeta) \text { such that } \frac{\partial\left(e_{i}\right)_{0}}{\partial z}=\sum_{j}\left(e_{j} D_{j i}(z, \zeta)\right)_{0} .
$$

Now assume that the $e_{i}$ 's are chosen so that $e_{1}, \ldots, e_{m-n}$ form a basis of $Z^{\prime}$; then the $\left(e_{i}\right)_{0}, i=1, \ldots, m-n$, are divisible by $h$, to order $(m-n)$ in $z$. The same is then true of $\partial\left(e_{i}\right)_{0} / \partial z$, to order $(m-n-1) ; D$ thus has the block decomposition with respect to $X=Z^{\prime} \oplus Z^{\prime \prime}$ :

$$
\left[\begin{array}{cc}
D^{\prime} & D^{\prime \prime} \\
O\left(z^{m-n-1}\right) & D^{\prime \prime \prime}
\end{array}\right]
$$

Referring to (2.35), similar decompositions hold for $C_{1}, C_{2}$, and so for the connection matrix $\Gamma=D+\frac{1}{2} C_{1}+\zeta C_{2}$. Finally, as in [Hi2, p. 178], the polar part of $\Gamma$ has the block diagonal form

$$
\frac{(m-n-1)}{2 z}\left[\begin{array}{ll}
1 & 0 \\
0 & 0
\end{array}\right]
$$

These facts then imply that one can find a change of basis matrix $S$ of the form

$$
\left[\begin{array}{ll}
z^{-(m-n-1) / 2} & 0 \\
0 & 1
\end{array}\right] \cdot[\mathbb{1}+O(z)]
$$

from this basis to a $\nabla$-flat basis, with the $O(z)$ term having the appropriate block form. Expressing $\underline{A}(z, \zeta)$ with respect to the $\nabla$-flat basis, one obtains (referring to $(2.35)$

$$
A(z, \zeta)=\left[\begin{array}{cc}
\alpha(\zeta) / z+\alpha^{\prime}(\zeta)+O(z), & O\left(z^{(m-n-1) / 2}\right) \\
O\left(z^{(m-n-1) / 2}\right), & \gamma(\zeta)+O(z)
\end{array}\right],
$$

which completes the proof that the boundary behaviour of our solutions to Nahm's equations is that given in condition B-2.

2f) Real Structure. We now have a solution $T_{i}(z)$ to Nahm's equations, satisfying the right boundary conditions; the last step is to show that the solution can be made skew adjoint. As before, this is done in several steps:

i) One defines a positive definite hermitian form on $X$.

ii) One shows that the endomorphisms $\underline{T}_{i}$ are skew adjoint with respect to this form.

iii) One shows that the connection preserves the form, and so the matrices $T_{i}(z)$ can be made skew adjoint.

iv) One must show that the boundary conditions and the hermitian structure are compatible, i.e. that the block decomposition of $T_{i}(z)$ at $z=0$ can be obtained in an orthonormal basis. 
Step i), the definition of the form, is an adaptation of that of Hitchin [Hi2, p. 179]. Let $s, t \in X_{z} \approx H^{0}\left(S_{p}, L^{z}(m+n-1)[-D]\right)$; the real structure pulls back $L^{z}(m+n-1)[-D]$ to $L^{-z}(m+n-1)[-\tau(D)]$; there is an antilinear map

$$
\sigma \cdot H^{0}\left(S_{p}, L^{z}(m+n-1)[-D]\right) \rightarrow H^{0}\left(S_{p}, L^{-z}(m+n-1)[-\tau(D)]\right) .
$$

$s \cdot \sigma(t)$ is a section of $H^{0}\left(S_{p}, \mathcal{O}(2 m+2 n-2)[-D-\tau(D)]\right)$; remember that $D \cup \tau(D)=$ $S_{p} \cap S_{p-1}$; thinking of $s \cdot \sigma(t)$ as a section of $\mathcal{O}(2 m+2 n-2)$ over $T \mathbb{P}_{1}(2.11)$, this means that there exist $a \in H^{0}\left(T \mathbb{P}_{1}, \mathcal{C}(2 n-2)\right), b \in H^{0}\left(T \mathbb{P}_{1}, \mathcal{O}(2 m-2)\right.$, with:

Therefore, over $S_{p}$ :

$$
s \cdot \sigma(t)=a g+b h .
$$

and over $S_{p-1}$ :

$$
s \cdot \sigma(t)=b h
$$

$$
s \cdot \sigma(t)=a g .
$$

By (2.16), and (1.2), $b$ decomposes uniquely as $b_{0} \eta^{m-1}+b_{1}(\zeta) \eta^{m-2}+\cdots$ $+b_{m-1}(\zeta)$, with $b_{i}$ of degree $2 i$. One then sets

$$
\langle s, t\rangle_{z}=b_{0}
$$

Once $\langle$,$\rangle has been defined, steps ii) and iii) are proven exactly as in Hitchin$ [Hi2], and so will not be repeated here.

We now consider step iv). In the case $m>n$, what must be shown is that the decomposition $X=Z^{\prime} \oplus Z^{\prime \prime}\left(\xi_{0}\right)$ is orthogonal at $z=0$, to order $(m-n) / 2$ in $z$. Let $s$ be a local $\nabla$-flat section of $Z^{\prime}$; then $s^{\prime}=z^{(m-n) / 2} s$ is a smooth section of $X$, and $s^{\prime}=h p^{\prime}$ over $T \mathbb{P}_{1}$ to order $(m-n)$ in $z$, for some $p^{\prime}$. Let $t$ be a local section of $Z^{\prime \prime}\left(\xi_{0}\right)$. Using the fact that $s^{\prime}=z^{-n} \rho(\tilde{s}), t=z^{-n} \rho(\tilde{t})$, it is fairly easy to see that $s^{\prime} \sigma(t)$ has no terms of order $\geqq(m+n-1)$ in $\eta$, to order $(m-n)$ in $z$; therefore, to order $(m-n)$ in $z$, one has

$$
s^{\prime} \sigma(t)=h p^{\prime} \sigma(t)
$$

and so, to order $(m-n)$ in $z$, the $\left(\operatorname{order}(m-1)\right.$ term in $\eta$ of $\left.b=p^{\prime} \sigma(t)\right)$ is the (order $(m+n-1)$ term in $\eta$ of $s^{\prime} \sigma(t)$, which is zero.

Finally, we check compatibility at the boundary, and positivity. We note that one has

$$
\begin{array}{r}
s, t \in H^{0}\left(S_{p}, L^{\mu_{p}-t}\left(m_{p}+m_{p-1}-1\right)\left[-S_{p, p-1}\right]\right) \\
\approx H^{0}\left(S_{p}, L^{\mu_{p}-t}\left(m_{p}+m_{p+1}-1\right)\left[-S_{p, p+1}\right]\right)
\end{array}
$$

using the section $f_{p}$ of (1.8). One then sees that

$$
\begin{gathered}
s \cdot \sigma(t) \in H^{0}\left(S_{p}, \mathcal{O}\left(2 m_{p}+2 m_{p-1}-2\right)\left[-S_{p} \cap S_{p-1}\right]\right) \\
\quad=H^{0}\left(S_{p}, \mathcal{O}\left(2 m_{p}+2 m_{p+1}-2\right)\left[-S_{p} \cap S_{p+1}\right]\right) .
\end{gathered}
$$

The technique used above therefore gives two possible forms $\langle,\rangle_{z},\{,\}_{z}$; it is easy to check that $\langle,\rangle_{z}=e_{p}\{,\}_{z}$, where $e_{p}$ is the constant of $C-4$ in the introduction normalised so that $\left|e_{p}\right|=1$; one then has $e_{p}=-(-1)^{m_{p}+m_{p-1}}$.

We now check that the definition of $\langle$,$\rangle is compatible with the glueing of Y_{0}$ and $X_{0}$ at $z=0$. Without loss of generality, set $m \geqq n$. Let elements $s, t$ of $Y_{0} \cong$ $H^{0}\left(S_{p-1}, \mathcal{O}(m+n-1)[-D]\right)$ be represented, using $(2.16)$, by polynomials of degree 
less than $n$ in $\eta$ :

$$
\begin{aligned}
\langle s, t\rangle_{Y_{0}} & =\text { degree }(n-1) \text { term (in } \eta) \text { of a } \\
& =- \text { degree }(m-1) \text { term (in } \eta) \text { of } b \text {, by }(2.41) \\
& =-\{s, t\}_{X_{0}} \\
& =(-1)^{m_{p}+m_{p-1}}\langle s, t\rangle_{X_{0}} .
\end{aligned}
$$

Now let us show that the form is definite. We begin on the interval $\left(\mu_{2}, \mu_{1}\right)$. The form is preserved by the connection, and is compatible with the $T_{i}$; in particular, at $\mu_{1}$, the residues of $T_{i}$ define an irreducible representation of $s u(2)$; these residues are skew hermitian with respect to $\langle$,$\rangle , which forces the form to be definite. In$ fact, one can compute its sign to be $(-1)^{m_{1}}$, from the basis of $X_{\mu_{1}}$, using the description of (2.21). Transporting to $\mu_{2}$, one has the same form there on $Y_{\mu_{2}}$. If $m_{2} \leqq m_{1}$, this form transfers to a definite form on $X_{\mu_{2}} \subset Y_{\mu_{2}}$, of $\operatorname{sign}(-1)^{m_{2}}$, by (2.43). If $m_{2}>m_{1}$, then $X_{\mu_{2}}=Z_{\mu_{2}}^{\prime} \oplus Z_{\mu_{2}}^{\prime \prime}$ as in (2.21); on $Z_{\mu_{2}}^{\prime \prime}$. the sign of the form is $(-1)^{m_{2}}$, by "transfer" from $Y_{\mu_{2}}$, above; on $Z_{\mu_{2}}^{\prime}$, explicit computation from (2.21) gives that the sign is also $(-1)^{m_{2}}$. Continuing in this way, one checks positivity on each interval in turn.

\section{From Nahm's Equations to Spectral Data}

In this section, we show that a generic solution $A(t, \zeta)$ to Nahm's equations, satisfying conditions $B$, yields back the spectral data. From $A(t, \zeta)$, we must obtain:

i) real curves $S_{p}$, of degree $2 m_{p}$, for $p=1, \ldots, N-1$,

ii) the partition of $S_{p} \cap S_{p-1}$ into $S_{p, p-1}$ and $S_{p-1, p}$,

iii) the section $\xi_{p}$ in $H^{0}\left(S_{p}, L^{\mu_{p+1}-\mu_{p}}\left(m_{p-1}+m_{p+1}\right)\left[-S_{p, p+1}-S_{p, p-1}\right]\right)$,

iv) the fact that $S_{p}$ satisfies the vanishing theorem (1.17). by

Part i) of this data is easy to obtain: let $S_{p}$ be the curve in $T \mathbb{P}_{1}$ defined

$$
\operatorname{det}(\eta 1-A(t, \zeta))=0
$$

for any $t \in\left(\mu_{p+1}, \mu_{p}\right) ; S_{p}$ is thus the spectrum of $A(t, \zeta)$ (recall that Nahm's equations are isospectral). As the $T_{i}$ 's are skew adjoint, $\zeta^{2} \overline{A\left(-\bar{\zeta}^{-1}\right)}=-A(\zeta)$, and so $S_{p}$ is real.

To obtain parts ii), iii) and iv) of the data, we begin by inverting the procedure used in Chap. 2 to obtain $A(t, \zeta)$; here, therefore, from the flow $A(t, \zeta)$, we obtain the appropriate flow $K_{t}$ of line bundles over the curves. Recall that in Chap. 2, $A(t, \zeta)=A_{0}(t)+A_{1}(t) \zeta+A_{2}(t) \zeta^{2}$, for $t \in\left(\mu_{p+1}, \mu_{p}\right)$, was derived from the exact sequence

$$
\begin{gathered}
0 \rightarrow H^{0}\left(S_{p}, L^{-t}(-1) \otimes E /\left(E_{p}^{+}+E_{N-p}^{-}\right)\right) \otimes \mathcal{O}(-2) \stackrel{\eta-A(t, \zeta)}{\longrightarrow} \\
H^{\mathrm{O}}\left(S_{p}, L^{-t}(-1) \otimes E /\left(E_{p}^{+}+E_{N-p}^{-}\right)\right) \otimes \mathcal{O} \stackrel{\mathrm{ev}}{\longrightarrow} L^{-t}(-1) \otimes E /\left(E_{p}^{+}+E_{N-p}^{-}\right) \rightarrow 0,
\end{gathered}
$$

where ev is the evaluation map. More succinctly:

$$
0 \rightarrow \mathcal{O}(-2)^{\oplus m_{p}} \rightarrow \mathcal{O}^{\oplus m_{p}} \rightarrow L^{-t}(-1) \otimes E /\left(E_{p}^{+}+E_{N-p}^{-}\right) \rightarrow 0 .
$$


Generically, of course, $E /\left(E_{p}^{+}+E_{N-p}^{-}\right)$is the restriction to $S_{p}$ of $L^{\mu_{p}}\left(m_{p}+m_{p-1}\right)$ $\left[-S_{p-1, p}\right]$. We now invert this procedure. Let $\eta$ also denote $\eta 1$. Define

$$
K_{t}=\operatorname{coker}(\eta-A(t, \zeta)): \mathcal{O}(-2)^{\oplus m_{p}} \rightarrow \mathcal{O}^{\oplus m_{p}} .
$$

Note that $K_{t}$ is not necessarily locally free over $S_{p}$; it can have torsion at eigenvalues of higher multiplicity. One way to smooth it out is to take a dual, which is torsion free; one has, over $S_{p}$,

$$
K_{t}^{*} \simeq \operatorname{ker}(\eta-A(t, \zeta))^{T}: \mathcal{O}^{\oplus m_{p}} \rightarrow \mathcal{O}(2)^{\oplus m_{p}} .
$$

Let the suffix adj denote the classical adjoint:

$$
(\eta-A(t, \zeta))^{T}(\eta-A(t, \zeta))_{\mathrm{adj}}^{T}=\operatorname{det}(\eta-A(t, \zeta)) \rrbracket .
$$

One has, over $S_{p}$ :

$$
\operatorname{Im}(\eta-A(t, \zeta))_{\mathrm{adj}}^{T} \subset K_{t}^{*} .
$$

When $(\eta-A(t, \zeta))$ has corank one $(\eta-A(t, \zeta))_{\text {adj }}^{T}$ is of rank one, and one has equality in (3.7); $K_{t}$ is then a line bundle. When the corank of $(\eta-A(t, \zeta))$ is greater than one, the classical adjoint vanishes.

To begin, we show that the flow of bundles is in the right direction. Let $s$ be a (meromorphic) section of $K_{t}^{*}$, for $t=t_{0}$; let $s$ be represented over $U_{0}=\{\zeta \neq \infty\}$ by $u$, and over $U_{1}=\{\zeta \neq 0\}$ by $v$; let $g\left(t_{0}\right)$ be a transition function for $K_{t_{0}}^{*}$; then $u=g\left(t_{0}\right) v$. One has over $U_{0}$,

$$
\left(\eta-A\left(t_{0}, \zeta\right)\right)^{T} u=0
$$

and over $U_{1}$

$$
\zeta^{-2}\left(\eta-A\left(t_{0}, \zeta\right)\right)^{T} v=0
$$

Let $A_{\#}(t, \zeta)=A_{1}(t) / 2+A_{2}(t) \zeta$; varying $t$, we ask that, as $t$ varies:

$$
\frac{\partial u}{\partial t}=A_{\#}^{T} u
$$

then, using Nahm's equations,

$$
\frac{\partial}{\partial t}(\eta-A)^{T} u=A_{\#}^{T}(\eta-A)^{T} u
$$

and so, if $u$ is a solution to $(3.8),(\eta-A)^{T} u$ is also; as the initial condition for this linear equation is $(\eta-A)^{T} u=0$, then $(\eta-A)^{T} u=0$ for all $t$.

Similarly, one can ask that

$$
\frac{d v}{d t}=-\left(A / \zeta-A_{\#}\right)^{T} v
$$

ensuring that $\zeta^{-2}(\eta-A)^{T} v=0$ for all $t$; then,

$$
A_{\#}^{T} u=\frac{\partial u}{\partial t}=\frac{\partial g}{\partial t} v-g\left(A / \zeta-A_{\#}\right)^{T} v
$$

and so 
Integrating,

$$
\frac{\eta}{\zeta} u=\frac{A}{\zeta} u=\frac{\partial g}{\partial t} g^{-1} u
$$

therefore,

$$
g(t, \eta, \zeta)=e^{t \eta / \zeta} g\left(t_{0}, \eta, \zeta\right)
$$

$$
K_{t}^{*}=K_{t_{0}}^{*} \otimes L^{\left(t-t_{0}\right)} .
$$

The flow $K_{t}^{*}$ is, at least, in the right direction; to identify $K_{t}$, one must examine the boundary behaviour of $A(t, \zeta)$ at the points $\mu_{p}$. In the study of boundary behaviour, we readopt the notation of Sect. 2. We begin with the case $m>n$.

Set $k=m-n$. Then, near $z=0$, one has, for $z>0$, the block decomposition:

$$
A^{T}(z, \zeta)=\left(\begin{array}{ll}
\alpha^{T}(z, \zeta) z^{-1}, & \delta^{T}(z, \zeta) z^{(k-1) / 2} \\
\beta^{T}(z, \zeta) z^{(k-1) / 2}, & \gamma^{T}(z, \zeta)
\end{array}\right) .
$$

$A^{T}$ is $m \times m, \alpha^{T}$ is $k \times k ; \alpha, \beta, \gamma, \delta$ are quadratic in $\zeta$, and are written $\alpha(z, \zeta)=\alpha_{0}(z)+$ $\alpha_{1}(z) \zeta+\alpha_{2}(z) \zeta^{2}$, etc. Let $\alpha^{T}(0, \zeta)=a(\zeta), \beta^{T}(0, \zeta)=b(\zeta), \gamma^{T}(0, \zeta)=c(\zeta), \delta^{T}(0, \zeta)=d(\zeta)$. One also had, for $z<0$, an $n \times n$ solution $A^{T}(z, \zeta)$, with $A^{T}(0, \zeta)=c(\zeta)$. Conjugating (3.10) by $\operatorname{diag}\left(z^{(k-1) / 2}, 1\right)$, one gets

$$
\left(\begin{array}{ll}
\alpha^{T}(z, \zeta) z^{-1} & \delta^{T}(z, \zeta) z^{k-1} \\
\beta^{T}(z, \zeta) & \gamma^{T}(z, \zeta)
\end{array}\right) .
$$

Recall that from $a(\zeta)=a_{0}+a_{1} \zeta+a_{2} \zeta^{2}$, one defined an irreducible $k$-dimensional representation of $\operatorname{sl}(2, \mathbb{C})$. For each $\zeta, a(\zeta)$ has a one dimensional kernel; one can choose a basis $e_{i}$ so that $\operatorname{ker} a(\zeta)=\left(\zeta^{k-1}, \zeta^{k-2}, \ldots, 1\right)^{T}$; in this basis, for example, $a(0) e_{i}=e_{i+1}$. We compute a section of $\operatorname{ker}(\eta-A)^{T}$, or equivalently, (as we shall see) a section of $\operatorname{Im}(\eta-A)_{\mathrm{adj}}^{T}$. We do this first at $\zeta=0$; it suffices to compute the first column of $(\eta-A)_{\mathrm{adj}}^{T}$, i.e., the minors along the top row. We find:

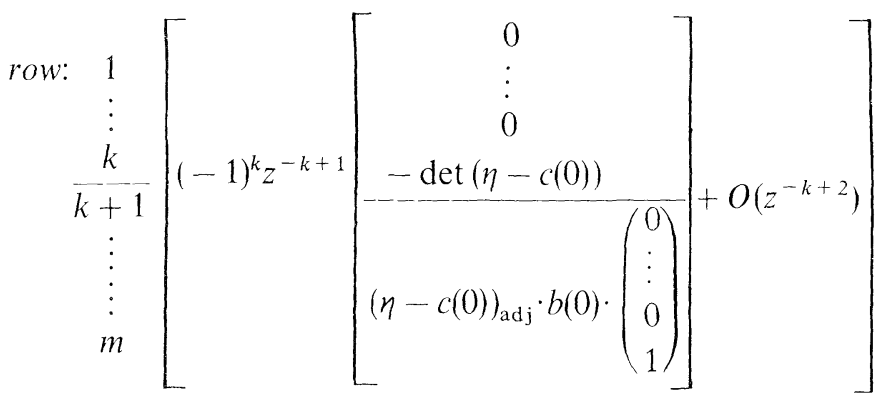

Multiplying through by $(-1)^{k} z^{-k+1}$, one obtains a non-zero limit vector, at $z=0$. Similarly, when $\zeta$ is arbitrary, one has the limit section of $\operatorname{ker}(\eta-A)^{T}$,

$$
\left(\frac{f_{1}(\eta, \zeta)}{f_{2}(\eta, \zeta)}\right)=\left(\frac{\operatorname{det}(\eta-c(\zeta)) \cdot 1_{k \times k}}{(\eta-c(\zeta))_{\mathrm{adj}} \cdot b(\zeta)}\right)\left(\begin{array}{c}
\zeta^{k-1} \\
\zeta^{k-2} \\
\vdots \\
\vdots \\
1
\end{array}\right)
$$


Note that, at $\zeta=0$, if one computes the $(k+i)^{t h}$ column of $(\eta-A)_{\mathrm{adj}}^{T}$, one obtains for the first $k$ entries, the finite limit at $z=0$,

$$
e_{1}^{i}(\eta, 0)^{T}=\left(0, \ldots, 0,\left[(1,0, \ldots, 0) d(0)(\eta-c(0))_{\mathrm{adj}}\right]_{i}\right)^{T}
$$

similarly, as $\zeta$ varies, one has a finite limit $e_{1}^{i}(\eta, \zeta)$. As rank $(\eta-A(z, \zeta))_{\mathrm{adj}}^{T} \leqq 1$ over $S_{p}$, the $(k+i)^{t h}$ column and the first column of $A$ are proportional; at the limit $z=0$, the factor of proportionality normalised by $-(-z)^{k+1}$ is $e_{1}^{i} / f_{1}$. As $f_{2}$ is finite, the limit vector $e_{2}^{i}$ of the last $n$ entries in the $(k+i)^{\text {th }}$ column is finite, when $\operatorname{det}(\eta-c(\zeta)) \neq 0$. Hartogs' theorem (in the variables $\zeta, z$ over $S_{p} \times \mathbb{C}$ ) then forces $e_{2}^{i}$ to be finite even when $\operatorname{det}(\eta-c(\zeta))=0$. We write the limit of the $(k+i)^{t h}$ column at $\zeta=0$ as:

$$
\left(e_{1}^{i}, e_{2}^{i}\right)^{T}=\left(0, \ldots, 0,\left((1,0, \ldots, 0) d(0)(\eta-c(0))_{\mathrm{adj}}\right)_{i}, e_{21}^{i}, \ldots, e_{2 n}^{i}\right)^{T} .
$$

Furthermore, one has, for some $p(\eta)$,

$$
\operatorname{det}(\eta-A(0))=\operatorname{det}(\eta-c(0)) \cdot p(\eta)-(1,0 \cdots 0) d(0)(\eta-c(0))_{\mathrm{adj}} b(0)(0 \cdots 0,1)^{T},
$$

which relates the equation of $S_{p}(\operatorname{det}(\eta-A(0))=0)$ and that of $S_{p-1}(\operatorname{det}(\eta-c(0))=0)$ over $\zeta=0$. Now suppose that we are in the generic case. Genericity precludes intersections of the spectral curves at multiple points; at $S_{p} \cap S_{p-1}$, therefore, $(\eta-A)_{\mathrm{adj}}^{T}$ is of rank one. On the other hand, away from the intersections, over $S_{p}$, where $\operatorname{det}(\eta-c(\zeta)) \neq 0$, (3.13) shows us that $(\eta-A)_{\mathrm{adj}}^{T}$ is again non-zero, and so $(\eta-A)$ has everywhere corank one on $S_{p}$. Similarly, changing $\zeta$-coordinates if necessary, from (3.16) one sees that $(\eta-c(\zeta))_{\text {adj }}$ is non-zero on $S_{p-1}$ away from $S_{p}$, and so $(\eta-c(\zeta))$ has everywhere corank one. Therefore, the sheaves $K_{0}$, on $S_{p}$ and $S_{p-1}$ are line bundles.

We now identify the bundles $K_{z}$ at $z=0$.

Proposition (3.17). Let $m>n$. Suppose that the Nahm data is generic. There is a partition of $S_{p} \cap S_{p-1}$ into divisors $D, \tau(D)$ such that, over $S_{p}$ and over $S_{p-1}, K_{0} \simeq$ $\mathcal{O}(m+n-1)[-D]$.

Proof i). Over $S_{p}$. The column vector (3.13) defining a section of $K_{0}^{*} \subset \mathcal{O}^{\oplus m}$, has, as entries, polynomials of degree $\leqq(m+n-1)$; (3.13) can be thought of as a map $\mathcal{O}(-m-n+1) \rightarrow K_{0}^{*}$, or, dually, as a map

$$
K_{0} \rightarrow \mathcal{O}(m+n-1) .
$$

We must show that this map vanishes on an appropriate $D$, and only on $D$. Let $E$ be the divisor cut out on $S_{p}$ by $S_{p-1}$; one wants $D+\tau(D)=E$. The $f_{1}$ portion of (3.13) vanishes on $E$, as $S_{p-1}$ is defined by $\operatorname{det}(\eta-c(\zeta))=0 ; f_{1}$ will vanish on any $D<E$. Set $f_{2}^{*}(\eta, \zeta)=\zeta^{m+n-1} \overline{f_{2}(\tau(\eta, \zeta))}=\zeta^{m+n-1} \overline{f_{2}}\left(-\bar{\eta} / \overline{\bar{\zeta}^{2}},-1 / \bar{\zeta}\right)$; consider the $n \times n$ rank 1 matrix $f_{2} f_{2}^{* T}$.

Lemma (3.19). There is a positive divisor $D$ such that $f_{2}$ vanishes on $D$ and $D+\tau(D)=E$ if and only if $f_{2} f_{2}^{* T}$ vanishes on $E$.

Proof. The proof in one direction is obvious; in the other, let $f_{2} f_{2}^{* T}$ vanish on $E$; then $f_{2, i} f_{2, j}^{*}$ vanishes on $E, \forall_{i, j}$. The fact that $E$ is real means that it can be written as $E=\Sigma m_{k}\left(p_{k}+\tau\left(p_{k}\right)\right)$, where $p_{k}$ are points of $S$. Let $f_{2, i}$ vanish at $p_{k}$ with 
multiplicity $g_{k, i}$, and at $\tau\left(p_{k}\right)$ with multiplicity $h_{k, i}$. As $f_{2, i} f_{2, j}^{*}$ is zero on $E, g_{k, i}+h_{k, j} \geqq m_{k}$ for all $i, j$. Set $g_{k}=\min \left(g_{k, i}\right), h_{k}=m_{k}-g_{k}$; then $f_{2, i}$ vanishes at $D=\Sigma g_{k} p_{k}+h_{k} \tau\left(p_{k}\right)$ for all $i$, and $D+\tau(D)=E$.

Returning to (3.17), to show that $f_{2} f_{2}^{* T}$ is zero over $E$, let $p \in S_{p} \cap S_{p-1}$. Changing coordinates on $T P_{1}$ if necessary, let $p$ be located at $\zeta=0$. One has from (3.13):

$$
f_{2}^{*}(\eta, \zeta)=\left(-\eta-\zeta^{2} \overline{\left.c\left(-\bar{\zeta}^{-1}\right)\right)_{\mathrm{adj}}} \zeta^{2} \overline{b\left(-\bar{\zeta}^{-1}\right)}\left(\begin{array}{c}
(-\zeta)^{-k+1} \\
\cdots \\
\cdots \\
\cdots \\
1
\end{array}\right) \zeta^{k-1}\right.
$$

as the $T_{i}(t)$ are skew adjoint, $\zeta^{2} \overline{c\left(-\bar{\zeta}^{-1}\right)}=-c(\zeta)^{T}, \zeta^{2} \overline{b\left(-\bar{\zeta}^{-1}\right)}=-d(\zeta)^{T}$; therefore at $\zeta=0$, up to a sign,

$$
f_{2} f_{2}^{* T}=(\eta-c(0))_{\mathrm{adj}} b(0)\left(\begin{array}{c}
0 \\
\ldots \\
\ldots \\
1
\end{array}\right)(1, \ldots, 0) d(0)(\eta-c(0))_{\mathrm{adj}}
$$

One now uses the fact that (3.12) and (3.15) are proportional over $S_{p}$; as we are at a point where $\operatorname{det}(\eta-c(0))=0$, this forces either $(\eta-c(0))_{\text {adj }} b(0)(0, \ldots, 1)^{T}=0$ or $(1,0, \ldots, 0) d(0)(\eta-c(0))_{\mathrm{adj}}=0$, by $(3.16)$.

The final step is to show that (3.18) vanishes only at $D$. This is done by remarking that $\operatorname{deg}\left(K_{0}\right)=\operatorname{deg}(O(m+n-1)[-D])=m(m-1)$.

ii) Over $S_{p-1}: K_{0}^{*}$ is defined over $S_{p-1}$ as the kernel of $(\eta-c(0))$; referring to (3.13), $f_{2}$ is a section of $K_{0}^{*}$. The proof that $K_{0} \sim \mathcal{O}(m+n-1)[-D]$ over $S_{p-1}$ is then just the repetition of that given over $S_{p}$.

We now analyse the case $m=n$. At $z=0$, one has limits $A^{+}(\zeta), A^{-}(\zeta)$ with $\operatorname{det}\left(\eta-A^{+}(\zeta)\right)=0, \operatorname{det}\left(\eta-A^{-}(\zeta)\right)=0$ defining $S_{p-1}, S_{p}$ respectively. Furthermore,

$$
A^{+}(\zeta)-A^{-}(\zeta)=\left(s_{0}+\zeta s_{1}\right)\left(\bar{s}_{1}-\zeta \bar{s}_{0}\right)^{T}=s(\zeta) s^{*}(\zeta)^{T}
$$

where $s_{0}, s_{1}$ are column vectors.

Again we define a section of $\operatorname{ker}\left(\eta-A^{+}(\zeta)\right)^{T}$ over $S_{p-1}$ and of $\operatorname{ker}\left(\eta-A^{-}(\zeta)\right)^{T}$ over $S_{p}$. Recall the "Weinstein-Aronzajn" relation:

$$
\operatorname{det}\left(\eta-A^{+}\right)^{T}=\operatorname{det}\left(\eta-A^{-T}-s^{*} s^{T}\right)=\operatorname{det}\left(\eta-A^{-T}\right)-s^{T}\left(\eta-A^{-}\right)_{\mathrm{adj}}^{T} s^{*} .
$$

Then, over $S_{p}$

$$
\left(\eta-A^{-}\right)^{T}\left(\eta-A^{-}\right)_{\mathrm{adj}}^{T} s^{*}=\operatorname{det}\left(\eta-A^{-}\right)^{T} S^{*}=0
$$

and, over $S_{p-1}$,

$$
\left(\eta-A^{+}\right)^{T}\left(\eta-A^{-}\right)_{\mathrm{adj}}^{T} S^{*}=\left(\eta-A^{-}\right)\left(\eta-A^{-}\right)_{\mathrm{adj}}^{T} s^{*}-s^{*} s^{T}\left(\eta-A^{-}\right)_{\mathrm{adj}}^{T} \mathrm{~s}^{*}=0,
$$

$\left(\eta-A^{-}(\zeta)\right)_{\mathrm{adj}}^{T} s^{*}$ thus defines a section of $K_{0}^{*}$, both over $S_{p}$ and $S_{p-1}$. Formula (3.21) shows us that, away from $S_{p} \cap S_{p-1},\left(\eta-A^{-}\right)_{\text {adj }}^{T}$ is non-zero on $S_{p}$; symmetri- 
cally, $\left(\eta-A^{+}\right)_{\text {adj }}^{T}$ is non-zero on $S_{p-1}$. The genericity condition then tells us that these are also both non-zero at the intersection. $K_{0}$ is then a line bundle, both on $S_{p}$ and on $S_{p-1}$.

Proposition (3.22). Let $m=n$. Suppose that the Nahm data is generic. There is a partition of $S_{p} \cap S_{p-1}$ into divisors $D, \tau(D)$ such that, over $S_{p}$ and over $S_{p-1}, K_{0} \simeq$ $\mathcal{O}(m+n-1)[-D]$.

Proof. As $\left(\eta-A^{-}(\zeta)\right)_{\mathrm{adj}}^{T} s^{*}$ is of degree $(2 m-1)$, as before, we have a map.

$$
K_{0} \rightarrow \mathcal{O}(2 m-1) \text {. }
$$

To show that this vanishes on the appropriate $D$, we use Lemma (3.19). It suffices to show that $\left(\eta-A^{-}(\zeta)\right)_{\mathrm{adj}}^{T} s^{*} s^{T}\left(\eta-A^{-}(\zeta)\right)_{\mathrm{adj}}^{T}$ vanishes on $S_{p} \cap S_{p-1}$. From (3.21), over $S_{p} \cap S_{p-1}, s^{T}\left(\eta-A^{-}(\zeta)\right)_{\mathrm{adj}}^{T} s^{*}=0$. As $\left(\eta-A^{-}(\zeta)\right)_{\mathrm{adj}}^{T}$ is of rank one over $S_{p-1}$, it can be written as a product $u w^{T}$, where $u, w$ are column vectors. Therefore, $s^{T} u w^{T} s^{*}=0$, and so either $s^{T} u=0$, or $w^{T} s^{*}=0$. Then, $\left(\eta-A^{-}\right)_{\text {adj }}^{T} s^{*}=0$ or $s^{T}\left(\eta-A^{-}\right)^{T}=0$, which yields the result. As above, one shows that it only vanishes on $D$.

One now has the necessary material to obtain parts ii) and iii) of the spectral data. $S_{p-1, p}$, of course, is just the divisor $D$ of the above propositions. As for part iii) consider the flow $K_{t}$ of bundles over $S_{p}$ for $t \in\left[\mu_{p+1}, \mu_{p}\right]$

$$
\begin{aligned}
\text {-at } t & =\mu_{p+1}, \text { one has } K_{\mu_{p+1}} \simeq \mathcal{O}\left(m_{p}+m_{p+1}-1\right)\left[-S_{p, p+1}\right] \\
\text { - -at } t & =\mu_{p}, K_{\mu_{p}}=\mathcal{O}\left(m_{p}+m_{p-1}-1\right)\left[-S_{p-1, p}\right] .
\end{aligned}
$$

One knows however, that $K_{\mu_{p}} \sim L^{\mu_{p+1}-\mu_{p}} K_{\mu_{p+1}}$; this can be written as an isomorphism

$$
\begin{aligned}
\mathcal{O} & \sim L^{\mu_{p+1}-\mu_{p}}\left(m_{p+1}-m_{p-1}\right)\left[-S_{p, p+1}+S_{p-1, p}\right] \\
& \sim L^{\mu_{p+1}-\mu_{p}}\left(m_{p+1}+m_{p-1}\right)\left[-S_{p, p+1}-S_{p, p-1}\right]
\end{aligned}
$$

as $\mathcal{O}\left(2 m_{p-1}\right) \sim\left[+S_{p-1, p}+S_{p, p-1}\right]$. This isomorphism is the section $\xi_{p}$ of iii).

For part iv), one must show that $H^{0}\left(S_{p}, K_{t}(-1)\right)=0$, whenever $A(t, \zeta)$ is finite. By the definition (3.4) of $K_{t}$ as a sheaf over $T \mathbb{P}_{1}$, one has the exact sequence,

$$
\begin{gathered}
\cdots \rightarrow H^{0}\left(T \mathbb{P}_{1}, \mathcal{O}(-1)^{\oplus m_{p}}\right) \rightarrow H^{0}\left(S_{p}, K_{t}(-1)\right) \rightarrow \\
H^{1}\left(T \mathbb{P}_{1}, \mathcal{O}(-3)^{\oplus m_{p}}\right) \stackrel{{ }^{=}=\eta-A(t, \zeta)}{\longrightarrow} H^{1}\left(T \mathbb{P}_{1}, \mathcal{O}(-1)^{\oplus m_{p}}\right),
\end{gathered}
$$

$H^{0}\left(T \mathbb{P}_{1}, \mathcal{O}(-1)^{\oplus m_{p}}\right)=0$; referring to the explicit form of $H^{1}\left(T \mathbb{P}_{1}, \mathcal{O}(-j)\right)$ given in Lemma (1.2), the map $F$ is injective. Therefore, $H^{0}\left(S_{p}, K_{t}(-1)\right)=0$.

To prove the positivity condition $C-4$, one considers both ends of the interval $\left(\mu_{p+1}, \mu_{p}\right)$; one has, at $\mu_{p+1}$, the section $f\left(\mu_{p+1}\right)$ of $\mathcal{O}\left(m_{p}+m_{p+1}\right)\left[-S_{p, p+1}\right]$, and, at $\mu_{p}$, the section $\widetilde{f}\left(\mu_{p}\right)$ of $\mathcal{O}\left(m_{p-1}+m_{p}\right)\left[-S_{p-1, p}\right]$, given by (3.13) or (3.21). One then "propagates" $f$ from $\mu_{p+1}$ to $\mu_{p}$, using Eq. (3.8), obtaining $f\left(\mu_{p}\right)$. Setting $v_{p}=f\left(\mu_{p}\right) / \widetilde{f}\left(\mu_{p}\right)$, one then must compute

$$
e_{p}=v_{p}^{*} v_{p}\left(g_{p-1} / g_{p+1}\right)=\frac{f\left(\mu_{p}\right)^{* T} f\left(\mu_{p}\right) g_{p-1}}{\tilde{f}\left(\mu_{p}\right)^{* T} \widetilde{f}\left(\mu_{p}\right) g_{p+1}} .
$$


However, from (3.8), $\partial_{t}\left(f^{* T} f\right)=0$, and so:

$$
e_{p}=\frac{f\left(\mu_{p+1}\right)^{* T} f\left(\mu_{p+1}\right)}{g_{p+1}} \frac{g_{p-1}}{\tilde{f}\left(\mu_{p}\right)^{* T} \widetilde{f}\left(\mu_{p}\right)},
$$

referring to the definitions, and keeping careful track of signs, one obtains $-(-1)^{m_{p}+m_{p-1}} e_{p}>0$.

\section{Reconstructing the Monopole}

4a) Introduction. In this section we explain the ADHMN construction and show how it associates a monopole to Nahm data. Starting with a solution $\left(T_{1}, T_{2}, T_{3}\right)$ to Nahm's equations, we show how to construct a rank $N$ bundle $C$ over $\mathbb{R}^{3}$ with connection and Higgs field $(\nabla, \Phi)$ which are regular and satisfy the Bogomolny equations. We then must show that it is a monopole, that is that $(\nabla, \Phi)$ satisfy the BPS boundary conditions.

We do this first in the generic case, when the Nahm data has associated spectral data to it and a holomorphic bundle $E$. From this, via twistor methods, one can construct a bundle $H$ on a dense open subset $U$ of $\mathbb{R}^{3}$, along with a connection and Higgs Field $(\tilde{\nabla}, \tilde{\Phi})$ which satisfy the Bogomolny equation. $U$ consists of the $x \in \mathbb{R}^{3}$ for which $\left.E\right|_{C_{x}}$ is holomorphically trivial (once we are done, we will see that $U=\mathbb{R}^{3}$ ). In Sect. 1.D, it was shown that $\mathbb{R}^{3}-U$ is compact, and that $(\tilde{\nabla}, \tilde{\Phi})$ satisfy the BPS coundary conditions.

We achieve our aim by defining an isomorphism $C \simeq H$ on a smaller open dense subset $\tilde{U}$ and by showing that $(\tilde{\nabla}, \tilde{\Phi}) \simeq(\nabla, \Phi)$ over $U$. One then has a global regular solution over all of $\mathbb{R}^{3}$, which satisfies the boundary conditions.

4b) The ADHMN Construction. For each interval $\left[\mu_{p+1}, \mu_{p}\right]$ let $\tilde{\mathscr{H}}_{p}$ be the Sobolev space of $L^{2}$ sections of $Y_{p}=X_{p} \otimes \mathbb{C}^{2}$ which have $L^{2}$ derivative. (The subscript $p$ denotes the interval.) By the Sobolev lemmas such sections are continuous. Similarly let $\mathscr{L}_{p}$ be the space of $L^{2}$ sections of $Y_{p}$.

At a boundary point $\mu_{p}$ of $\left[\mu_{p+1}, \mu_{p}\right]$, if $m_{p} \geqq m_{p-1}, \quad Y_{p}\left(\mu_{p}\right)=$ $Y_{p-1}\left(\mu_{p}\right) \oplus Y_{p-1}\left(\mu_{p}\right)^{\perp}$, and we adopt the terminology continuing for vectors in the first space and terminating for those in the second. If $m_{p} \leqq m_{p-1}$ all vectors of $Y_{p}$ are continuing; thus, continuing vectors on both sides are identified. Define similar terminology for the other end.

The Sobolev space $\mathscr{H}_{p}$ is defined to be the subspace of $\tilde{\mathscr{H}}_{p}$ consisting of sections whose terminating components at each end are zero. Define the operator $D_{p}(x)$, for $x \in \mathbb{R}^{3}$, by

$$
D_{p}(x): \mathscr{H}_{p} \rightarrow \mathscr{L}_{p}, \quad D_{p}(x)=i \nabla_{t}-\left(\underline{T}^{p}+i \underline{x}\right),
$$

where $\underline{T}^{p}=\Sigma T_{i} \otimes e_{i}$ and $\underline{x}=\Sigma x_{i}\left(1 \otimes e_{i}\right)$ for $\left(e_{1}, e_{2}, e_{3}\right)$ the unit imaginary quaternions. Because the components of the section acted on by the singular part of $T^{p}$ are zero this operator is well defined and has image in $\mathscr{L}_{p}$.

If $m_{p}=m_{p-1}$ the boundary condition for the solution of Nahm's equation implies that for some $x \in X_{p}\left(\mu_{p}\right)$ and some $\alpha \in \mathbb{C}^{2}$, 


$$
\underline{T}^{p}\left(\mu_{p}\right)-\underline{T}^{p-1}\left(\mu_{p}\right)=x \otimes x^{*} \otimes\left(\alpha \otimes \alpha^{*}-\langle\alpha, \alpha\rangle 1 / 2\right) \in \operatorname{End}\left(X_{p}\left(\mu_{p}\right)\right) \otimes s l(2, \mathbb{C}) .
$$

In such a case, let $W_{p}$ be the span of $x \otimes \alpha$ and let $\pi_{p}: Y_{p}\left(\mu_{p}\right) \rightarrow W_{p}$ be the orthogonal projection.

Define $\mathscr{H} \subset \bigoplus_{p=1}^{N-1} \mathscr{H}_{p}$ to be the space of all sections $f=\left(f_{1}, \ldots, f_{N-1}\right)$ such that $f_{p}\left(\mu_{p}\right)=f_{p-1}\left(\mu_{p}\right)$. Nahm's operator is defined to be

$$
\begin{gathered}
\mathscr{D}(x): \mathscr{H} \rightarrow \mathscr{L}=\left(\bigoplus_{p=1}^{N-1} \mathscr{P}_{p}\right) \oplus\left(\underset{\substack{q \\
m_{q}=m_{q-1}}}{\bigoplus} W_{q}\right) \\
f: \mapsto\left\{\left[\left(D_{1}(x) f_{1}, \ldots, D_{N-1}(x) f_{N-1}\right)\right],\left[\left(\pi_{q_{1}} f_{q_{1}}\left(\mu_{q_{1}}\right), \ldots, \pi_{q_{r}} f_{q_{r}}\left(\mu_{q_{r}}\right)\right]\right\},\right.
\end{gathered}
$$

where $q_{1}, \ldots, q_{r}$ are all the indices for which the jump $m_{q}-m_{q-1}$ is zero.

Note that the kernel of Nahm's operator is all $\left(f_{1}, \ldots, f_{N-1}\right)$ such that

$-D_{p} f_{p}=0$

- - each $f_{p}$ is $L^{2}$

- the terminating components are zero

- the continuing components are continuous

-at zero jumps, $f_{q}\left(\mu_{q}\right)$ is in $W_{q}^{\perp}$.

Define

$$
D_{p}^{*}(x)=i \nabla_{t}+\left(\underline{T}^{p}+i \underline{x}\right)
$$

Then integrating by parts it is easy to deduce that the cokernel of $\mathscr{D}(x)$ is all $\left\{\left[g_{1}, \ldots, g_{N-1}\right],\left[w_{q_{1}}, \ldots, w_{q_{r}}\right]\right\}$ such that:

$-D_{p}^{*}(x) g_{p}=0$

$-g_{p}$ is in $L^{2}$

- the continuing components are continuous except at zero jumps, where

$$
g_{q}\left(\mu_{q}\right)-g_{q-1}\left(\mu_{q}\right)=w_{q} \in W_{q} .
$$

Notice that the terminating components of the $g_{p}$ are not constrained except by the $L^{2}$ requirement.

Let us call these boundary conditions for the kernel of $\mathscr{D}(x)$ and the cokernel of $\mathscr{D}(x)$ the Nahm and co-Nahm boundary conditions.

We define the "bundle" $C(x)$ by

$$
C(x)=\operatorname{coker} \mathscr{D}(x) \subset \mathscr{L} .
$$

We shall show, by calculating the index of $\mathscr{D}(x)$ and proving that dim $\operatorname{ker} \mathscr{D}(x)=0$, that rank $C(x)=N$, and so $C$ is in fact a bundle.

To do this we investigate the behaviour of solutions to $D_{p}^{*}(x) \varphi=0$ on an interval $\left(\mu_{p+1}, \mu_{p}\right)$. If $z=t-\mu_{p}$ is a parameter near $\mu_{p}$ and $k=m_{p}-m_{p-1}>0$, then the theory of singular, regular, ordinary differential equations (as used in Hitchin [Hi2]) tells us that the $2 m_{p}$ dimensional space of solutions to $D_{p}^{*}(x) \varphi=0$ decomposes into a direct sum of three pieces:

1) $A k-1$ dimensional space which are $O\left(z^{-(k-1) / 2}\right)$ near $\mu_{p}$;

2) $A k+1$ dimensional space which are $O\left(z^{(k-1) / 2}\right)$ near $\mu_{p}$; and

3) $A 2 m_{p-1}$ dimensional space of solutions which $O\left(z^{0}\right)$ at $\mu_{p}$.

The first two of these are terminating and the third is continuing. 
For each interval $\left[\mu_{p+1}, \mu_{p}\right], p=1, \ldots, N-1$, let $V_{p}$ be the $2 m_{p}$ dimensional kernel of $D_{p}^{*}$; let $V_{0}=V_{N}=\langle 0\rangle$. Define $U_{p} \subset V_{p} \times V_{p-1}$ for $p=1, \ldots, N$ to be the space of pairs satisfying the co-Nahm boundary conditions at $\mu_{p}$.

The analysis of the boundary behaviour shows that $\left(m_{0}=m_{N}=0\right)$

$$
\operatorname{dim} U_{p}=m_{p}+m_{p-1}+1 .
$$

Define a linear map

$$
\begin{gathered}
\chi: \bigoplus_{p=1}^{N} U_{p} \rightarrow \bigoplus_{p=1}^{N-1} V_{p} \\
\chi:\left(\left(0, \hat{u}_{1}\right),\left(u_{1}, \hat{u}_{2}\right),\left(u_{2}, \hat{u}_{3}\right), \ldots,\left(u_{N}, 0\right)\right) \mapsto\left(\hat{u}_{1}-u_{1}, \hat{u}_{2}-u_{2}, \ldots, \hat{u}_{N}-u_{N}\right),
\end{gathered}
$$

whose kernel is clearly the cokernel of $\mathscr{D}(x)$. We want to show that the cokernel of $\chi$ is dual to the kernel of $\mathscr{D}$. It will then follow that the index of $\mathscr{D}$ is $\sum_{p=1}^{N-1} 2 m_{p}-$ $\sum_{p=1}^{N}\left(m_{p-1}+m_{p}+1\right)=-N$.

Notice that because of the Liebniz rule if $D_{p} f=0$ and $D_{p}^{*} g=0$ then $\langle f, g\rangle$ is a constant in $t$. Hence $V_{p}^{*}$ can be identified with the space of all solutions to $D_{p} f=0$. For this equation we can repeat the analysis of boundary behaviour above and find a similar result, except that the dimensions $k-1$ and $k+1$ are interchanged.

Assume now that $\left(f_{1}, \ldots, f_{N-1}\right)$ belongs $\bigoplus_{p=1}^{N-1} V_{p}^{*}$ and annihilates the image of $\chi$. At $\mu_{1}$ if we apply $\chi$ to $\left(\left(0, u_{1}\right),(0,0) \cdots(0,0)\right)$, then $\left\langle f_{1}, u_{1}\right\rangle=0$ for all $u_{1}$ in the $m_{1}+1$ dimensional space of decaying solutions. Hence $f_{1}$ is in the $m_{1}-1$ dimensional space of decaying solutions to $D_{p} f_{1}=0$. At a typical point $\mu_{p}$ with say, $k=m_{p}-m_{p-1}>0$, if we take a pair $\left(u_{p-1}, \hat{u}_{p}\right)$ with $u_{p-1}\left(\mu_{p}\right)=\hat{u}_{p}\left(\mu_{p}\right)$ under the glueing then $0=\left\langle\chi\left((0,0) \cdots\left(u_{p-1}, \hat{u}_{p}\right), \ldots,(0,0)\right),\left(f_{1}, \ldots, f_{N-1}\right)\right\rangle=$ $\left\langle u_{p-1}, f_{p-1}\right\rangle-\left\langle\hat{u}_{p}, f_{p}\right\rangle=\left\langle u_{p-1}\left(\mu_{p}\right), f_{p-1}\left(\mu_{p}\right)-f_{p}\left(\mu_{p}\right)\right\rangle$, so the continuing components of $f_{p-1}, f_{p}$ match.

Next, if we take $u_{p-1}=0$ and $\hat{u}_{p}$ with zero continuing component and decaying terminating component, it follows that $f_{p}$ has decaying terminating component. If $k=0$ we can, in addition, choose $\hat{u}_{p}\left(\mu_{p}\right)-u_{p-1}\left(\mu_{p}\right) \in W_{p}$, so that $f_{p}\left(\mu_{p}\right)=f_{p-1}\left(\mu_{p}\right)$ and

$$
0=\left\langle\hat{u}_{p}\left(\mu_{p}\right)-u_{p-1}\left(\mu_{p}\right), f_{p}\left(\mu_{p}\right)\right\rangle .
$$

In all cases, $f$ satisfies the Nahm boundary conditions

Clearly, the converse is also true, if $\left(f_{1}, \ldots, f_{N-1}\right)$ is in the kernel of $\mathscr{D}$ it annihilates the cokernel of $\chi$. So we have proved:

Proposition 4.4. The index of Nahm's operator is $-N$.

It remains to prove a vanishing theorem for the kernel at $\mathscr{D}$. Using Nahm's equations, it is straightforward to calculate the Weitzenbock type formula

$$
D_{p}^{*}(x) D_{p}(x)=-\left((d /(d z))(d /(d z))+\left(\underline{T}^{p}+i \underline{x}\right)^{*}\left(\underline{T}^{p}+i \underline{x}\right) .\right.
$$

Hence if $D_{p}(x) f_{p}=0$, we have 


$$
\begin{aligned}
0 & =\int\left\langle\left(-\frac{d}{d z} \frac{d}{d z} f_{p}\right), f_{p}\right\rangle+\left\langle\left(\underline{T}^{p}+i \underline{x}\right) f_{p},\left(\underline{T}^{p}+i \underline{x}\right) f_{p}\right\rangle \\
& =\left\|\frac{d f_{p}}{d z}\right\|^{2}+\left\|\left(\underline{T}^{p}+i \underline{x}\right) f_{p}\right\|^{2}-\left\langle\frac{d f_{p}}{d z}, f_{p}\right\rangle\left(\mu_{p+1}\right)-\left\langle\frac{d f_{p}}{d z}, f_{p}\right\rangle\left(\mu_{p}\right) \\
& =\left\|\frac{d f_{p}}{d z}\right\|^{2}+\left\|\left(\underline{T}^{p}+i \underline{x}\right) f_{p}\right\|^{2}-\left\langle\left(\underline{T}^{p}+i \underline{x}\right) f_{p}, f_{p}\right\rangle\left(\mu_{p+1}\right)+\left\langle\left(\underline{T}^{p}+i \underline{x}\right) f_{p}, f_{p}\right\rangle\left(\mu_{p}\right) .
\end{aligned}
$$

If $\left(f_{1}, \ldots, f_{N-1}\right)$ is in the kernel of $\mathscr{D}(x)$ and there are no zero jumps, then the continuity of $f_{p}$ and $\underline{T}^{p}$ gives

$$
0=\sum_{p=1}^{N-1}\left\|\frac{d f_{p}}{d z}\right\|^{2}
$$

and hence $f_{p}=0$ for all $p$.

If there are zero jumps, this expression has additional terms of the form

$$
-\left\langle\left(\underline{T}^{p}-\underline{T}^{p-1}\right) f_{p}, f_{p}\right\rangle\left(\mu_{p}\right)
$$

but inspection of the boundary behaviour of $\underline{T}^{p}-\underline{T}^{p-1}$ shows that for $f_{p}\left(\mu_{p}\right) \in W_{p}^{\perp}$ this whole term is non-negative.

Therefore $\operatorname{ker} \mathscr{D}(x)=0$ and we have that $C(x)$ is a rank $N$ bundle on $\mathbb{R}^{3}$.

The connection and Higgs field for $C$ are defined by composing differentiation and multiplication by $i z$ with the orthogonal projection $\pi: \mathscr{L} \rightarrow C$ :

$$
\nabla_{i}=\pi_{\circ}\left(\partial / \partial x^{i}\right), \quad \Phi=\pi_{\circ} \mathrm{iz} .
$$

The same proof as that of [Hi2] shows that this defines a smooth solution to the Bogomoln'yi equations; in the next section we shall relate these constructions to the twistor approach for generic Nahm data.

4c) Link to the Twistor Approach. In the previous discussion we realized the cokernel of Nahm's operator $\mathscr{D}(x)$ as the kernel of an exact sequence

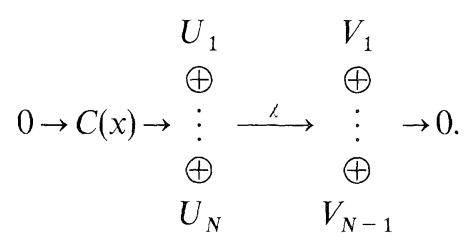

This should be a familiar sight to the reader by now! If we take a real section $C_{x}$ not intersecting any of the $S_{p, p+1}$ or contained in any spectral curve, then restricting 1.14 to this real section gives an exact sequence

$$
\begin{aligned}
& H^{0}\left(C_{x}, L^{\mu_{1}}\left(m_{1}\right)\right) \\
& \oplus \quad H^{0}\left(S_{1} \cap C_{x}, L^{\mu_{1}}\left(m_{1}\right)\right) \\
& H^{0}\left(C_{x}, L^{\mu_{2}}\left(m_{2}+m_{1}\right)\right) \\
& 0 \rightarrow H^{0}\left(C_{x}, E\right) \rightarrow \quad \rightarrow \quad H^{0}\left(S_{2} \cap C_{x}, L^{\mu_{2}}\left(m_{1}+m_{2}\right)\right) \\
& \text { : } \rightarrow \\
& \oplus \quad \oplus \\
& H^{0}\left(C_{x}, L^{\mu_{N}}\left(m_{N-1}\right)\right) \quad H^{0}\left(S_{N-1} \cap C_{x}, L^{\mu_{N-1}}\left(m_{n-1}+m_{N-2}\right)\right) .
\end{aligned}
$$


Our purpose is to identify this term by term with (4.6). This will give us $H^{0}\left(C_{x}, E\right)=$ coker $\mathscr{D}$ and identify the bundle obtained over $\mathbb{R}^{3}$ by this construction with that obtained by the twistor construction.

Notice first that because $L$ is trivial on $C_{x}$ we have

$$
\operatorname{dim} H^{0}\left(\left(C_{x}, L^{\mu_{p}}\left(m_{p}+m_{p-1}\right)\right)=m_{p}+m_{p-1}+1,\right.
$$

and because $S_{p} \cap C_{x}$ is $2 m_{p}$ points

$$
\operatorname{dim} H^{0}\left(S_{p} \cap C_{x}, L^{\mu_{p}}\left(m_{p}+m_{p-1}\right)\right)=2 m_{p} .
$$

Recall the vanishing theorem 1.17 which can easily be extended to

$$
\begin{array}{ll}
H^{0}\left(S_{p}, L^{\mu_{p}-t}\left(m_{p}+m_{p-1}+r\right)\left[-S_{p, p-1}\right]\right)=0 & \text { for } \quad r \leqq-2, \\
H^{1}\left(S_{p}, L^{\mu_{p}-t}\left(m_{p}+m_{p-1}+r\right)\left[-S_{p, p-1}\right]\right)=0 & \text { for } \quad r \geqq-2
\end{array}
$$

for $\mu_{p+1}<t<\mu_{p}$ with the appropriate results also at the boundary points. The Riemann-Roch theorem gives

$$
\begin{aligned}
& h^{0}\left(S_{p}, L^{\mu_{p}-t}\left(m_{p}+m_{p-1}+r\right)\left[-S_{p, p-1}\right]\right)-h^{1}\left(S_{p}, L^{\mu_{p}-t}\left(m_{p}+m_{p-1}+r\right)\left[-S_{p, p-1}\right]\right) \\
& \quad=(2+r) m_{p} .
\end{aligned}
$$

From the exact sequence on $\mathbb{P}_{1}$

$$
0 \rightarrow \mathcal{O}(-1) \rightarrow \mathcal{O}^{\oplus 2} \rightarrow \mathcal{O}(1) \rightarrow 0
$$

obtained by evaluating sections on $\mathbb{C}^{2} \simeq H^{0}\left(\mathbb{P}_{1}, \mathcal{O}(1)\right)$, and the vanishing theorem we have

$$
\begin{aligned}
H^{0}\left(S_{p}, L^{\mu_{p}-t}\left(m_{p}+m_{p-1}\right)\left[-S_{p . p-1}\right]\right) & =H^{0}\left(S_{p}, L^{\mu_{p}-t}\left(m_{p}+m_{p-1}-1\right)\left[S_{p, p-1}\right]\right) \otimes \mathbb{C}^{2} \\
& =X_{p}(t) \otimes \mathbb{C}^{2} .
\end{aligned}
$$

This space is then naturally identified with $Y_{p}(t)$. Also, from the vanishing theorem and the sequence $0 \rightarrow \mathcal{O}(-2) \rightarrow \mathcal{O} \rightarrow \mathcal{O}_{C_{x}} \rightarrow 0$,

$$
H^{0}\left(S_{p}, L^{\mu_{p}-t}\left(m_{p}+m_{p-1}\right)\left[-S_{p, p-1}\right]\right)=H^{0}\left(S_{p} \cap C_{x}, L^{\mu_{p}-t}\left(m_{p}+m_{p-1}\right)\left[-S_{p, p-1}\right]\right) .
$$

Over $C_{x}$ we can fix a " $C_{x}$-trivialization" of $L^{t}$ which is defined relative to the standard trivialisations over $U_{i}$ by the functions $\phi_{i}$,

$$
\begin{aligned}
& \phi_{0}(t, \zeta)=\exp \left(t\left(\left(x_{1}-i x_{2}\right) \zeta+x_{3}\right)\right), \\
& \phi_{1}(t, \zeta)=\exp \left(t \left(\left(-x_{3}+\left(x_{1}+x_{2}\right) / \zeta\right) .\right.\right.
\end{aligned}
$$

Evaluating with respect to this $C_{x}$ trivialization at these $2 m_{p}$ points fixes an isomorphism $H^{0}\left(S_{p} \cap C_{x}, L^{\mu_{p}-t}\left(m_{p}+m_{p-1}\right)\left[-S_{p, p-1}\right]\right)$ into a fixed $2 m_{p}$ dimensional space; composing with (4.7), one then has a $2 m_{p}$ dimensional space $V_{p}$ of sections of $Y_{p}$ defined by asking that the image be constant under this map.

Proposition 4.8. $\underline{V}_{p}$ is the kernel of $D_{p}^{*}(x)$, i.e., $\underline{V}_{p}=V_{p}$.

Proof. Start with a section $s$ of

$$
H^{0}\left(S_{p}, L^{\mu_{p}-t}\left(m_{p}+m_{p-1}\right)\left[-S_{p, p-1}\right]\right),
$$

which is some 


$$
s(t, \eta, \zeta)=p_{0}(\zeta) f_{0}(t, \eta, \zeta)=e^{\left(\mu_{p}-t\right) \eta / \zeta \zeta m_{p}+m_{p-1}} p_{1}(\zeta) f_{1}(t, \eta, \zeta) q(\eta, \zeta),
$$

where $q$ is the transition function for $\left[-S_{p-1, p}\right]$, the $f_{i}$ represent sections in $X_{p}(t)$ over $U_{i}$ and the $p_{i}(\zeta)$ represent sections of $\mathcal{O}(1)$.

If we restrict to $S_{p} \cap C_{x}=\left\{\left(\eta_{i}, \zeta_{i}\right), i=1, \ldots, 2 m_{p}\right\}$ we find that the coefficients of the section with respect to the $C_{x}$ trivialisation are

$$
p_{0}\left(\zeta_{i}\right) f_{0}\left(t, \eta_{i}, \zeta_{i}\right) e^{\left(\mu_{p}-t\right)\left(x_{3}+\left(x_{1}-i x_{2}\right) \zeta_{2}\right)}
$$

and these are required to be constant.

From the definition of the connection (2.6)

$$
\frac{d}{d z}=\nabla_{z}-i T_{3}+\zeta\left(T_{1}-i T_{2}\right)
$$

and moreover as we are on $S_{p} \cap C_{x}$ we have that

$$
\eta_{i}=x_{1}+i x_{2}-2 x_{3} \zeta_{i}+\left(\zeta_{i}\right)^{2}\left(x_{1}-i x_{2}\right)
$$

and

$$
\eta_{i}=T_{1}+i T_{2}+2 i T_{3} \zeta_{i}+\left(\zeta_{i}\right)^{2}\left(-T_{1}+i T_{2}\right)
$$

from the definition of the $T^{i}$ in Sect. 2 .

Combining all these and choosing a basis of $H^{0}\left(T \mathbb{P}_{1}, \mathcal{O}(1)\right)$ gives us

$$
\left[\left(\nabla_{t}+\underline{T}^{p}+i \underline{x}\right) s\right]\left(\eta_{i}, \zeta_{i}, t\right)=0 .
$$

But the isomorphism (4.7) then implies that

$$
\left(\nabla_{t}+\underline{T}^{p}+i \underline{x}\right) s=0
$$

as required.

We now have an isomorphism $V_{p} \simeq H^{0}\left(S_{p} \cap C_{x}, L^{\mu_{p}}\left(m_{p}+m_{p-1}\right)\right)$ and an embedding

$$
\begin{aligned}
& H^{0}\left(C_{x}, L^{\mu_{p}}\left(m_{p}+m_{p-1}\right)\right) \\
& \quad \rightarrow H^{0}\left(S_{p} \cap C_{x}, L^{\mu_{p}}\left(m_{p}+m_{p-1}\right) \oplus H^{0}\left(S_{p-1} \cap C_{x}, L^{\mu_{p}}\left(m_{p-1}+m_{p-2}\right)=V_{p} \oplus V_{p-1},\right.\right.
\end{aligned}
$$

the next step is to show that the image is $U_{p}$.

We start with the case of $m_{p}>m_{p-1}$. Recall the analysis above of the solutions about $\mu_{p}$. To make statements about the decay of sections we have to choose a trivialization of the bundle $Y$. We shall consider two different kinds. The first is a covariantly constant (using the connection on $X_{p}$ defined in $2 e$ and $Y_{p}=X_{p} \otimes \mathbb{C}^{2}$ ) trivialization, which we shall call a Nahm trivialization, and the second is a trivialization using sections of

$$
H^{0}\left(S_{p}, L^{\mu_{p}-t}\left(m_{p}+m_{p-1}\right)\left[-S_{p, p-1}\right]\right)
$$

which are holomorphic in $t$ and defined at $\mu_{p}$. This we shall call a holomorphic trivialization.

We saw above that, in a Nahm trivialisation, letting $k=m_{p}-m_{p-1}$ and setting $z=t-\mu_{p}$ the $2 m_{p}$ dimensional space of all solutions is a direct sum of three pieces:

A) $A k-1$ dimensional space of solutions blowing up like $z^{-(k-1) / 2}$, 
B) $A k+1$ dimensional space of solutions decaying like $z^{(k-1) / 2}$,

C) $A 2 m_{p-1}$ dimensional space of solutions which are of order $z^{0}$ at $\mu_{p}$.

If, as is more natural in the twistor picture, we use a holomorphic trivialization, the results of section $2 e$ imply that these spaces become:

A) $A k-1$ dimensional space of sections blowing up like $z^{-(k-1)}$,

B) $A k+1$ dimensional space of sections which are of order $z^{0}$ at $\mu_{p}$,

C) $A 2 m_{p-1}$ dimensional space of sections which are also of order $z^{0}$ at $\mu_{p}$.

The upshot of this is that to satisfy the co-Nahm boundary conditions a section in the holomorphic trivialization has to live in the sum of the B) and C) components on the "larger side" and also be continuous in the C) components.

First, the decay behaviour. From the results of Sect. $2 c$ ),

$$
Y_{p}\left(\mu_{p}\right) \subset H^{0}\left(S_{p}, \mathcal{O}\left(m_{p}+m_{p-1}\right)\left[-S_{p . p-1}\right]\right)
$$

breaks into three pieces:

$Y_{A}: A k-1$ dimensional space of sections of $\mathcal{O}\left(m_{p}+m_{p-1}\right)$ of the form $\rho_{x} g_{p-1} s$, where $\left(\rho_{x}=0\right)$ defines $C_{x}$ and $s$ is pulled back from $\mathbb{P}_{1}$.

$Y_{B}: A k+1$ dimensional space of sections of $\mathcal{O}\left(m_{p}+m_{p-1}\right)$ of the form $g_{p-1} s$ where $s$ is pulled back from $\mathbb{P}_{1}$.

$Y_{C}: A 2 m_{p-1}$ dimensional space of sections of $\mathcal{O}\left(m_{p}+m_{p-1}\right)$ which are of degree $m_{p-1}-1$ in $\eta$ and vanish on $S_{p-1, p}$.

Consider now the restriction maps

$$
\begin{aligned}
Y_{p}\left(\mu_{p}\right) \stackrel{\sigma}{\longrightarrow} & H^{0}\left(C_{x} \cap S_{p}, \mathcal{O}\left(m_{p}+m_{p-1}\right)\left[-S_{p, p-1}\right]\right) \\
& H^{0}\left(C_{x}, \mathcal{O}\left(m_{p}+m_{p-1}\right)\left[-S_{p, p-1}\right]\right) .
\end{aligned}
$$

For $t<\mu_{p}$ the map $\sigma$ in (4.13) is an isomorphism and for $t=\mu_{p}$ it clearly kills the space $Y_{A}$. Notice that the map $\rho$ is an inclusion because $H^{0}\left(\mathbb{P}_{1}, \mathcal{O}\left(-m_{p}+m_{p-1}\right)\right)=0$ when $m_{p}>m_{p-1}$. In addition we have

Proposition 4.14. The map $\sigma$ in (4.13) maps $Y_{B} \oplus Y_{C}$ isomorphically onto the image of the map $\rho$.

Proof. As, from $2 \mathrm{c}), H^{0}\left(T \mathbb{P}_{1}, \mathcal{O}\left(m_{p}+m_{p-1}\right) \otimes \mathscr{I}\left(-S_{p, p-1}\right)\right)=H^{O}\left(S_{p}, \mathcal{O}\left(m_{p}+m_{p-1}\right)\right.$ $\left.\left[-S_{p, p-1}\right]\right)$ then extending to $T \mathbb{P}_{1}$ everything in $\sigma\left(Y_{p}\left(\mu_{p}\right)\right)$ is in the image of the vertical map.

Let $s$ in $Y_{B} \oplus Y_{C}$ vanish on $C_{x} \cap S_{p}$. Divide $s$ by $\rho_{x} \in \Gamma(\mathcal{O}(2))$, lift to $T \mathbb{P}_{1}$, then restrict the result to $S_{p-1}$; the vanishing theorem tells us that the result is zero. $s$ is then divisible by $\rho_{x}$ and $g_{p-1}$, which contradicts the fact that its degree in $\eta$ is less than $m_{p-1}$.

The map is thus an injection and so, counting dimensions, an isomorphism.

Thus, at $\mu_{p}$, the isomorphism (4.7) fails. The values in $H^{0}\left(C_{x} \cap S_{p}, \mathcal{O}\left(m_{p}+m_{p-1}\right)\right.$ $\left.\left[-S_{p, p-1}\right]\right)$ that do correspond to solutions in $V_{p}$ are those in $\sigma\left(Y_{p}\left(\mu_{p}\right)\right.$; by Proposition (4.14), this is $H^{0}\left(C_{x}, \mathcal{O}\left(m_{p}+m_{p-1}\right)\left[-S_{p, p-1}\right]\right)$. 
As there are no constraints on decay behaviour on the "smaller" side, $H^{0}\left(C_{x}, \mathcal{O}\left(m_{p}+m_{p-1}\right)\left[-S_{p . p-1}\right]\right)$ therefore corresponds on both sides of $\mu_{p}$ to solutions with the right decay behaviour; to see that it also matches them up correctly we note that at the endpoints the glueing of the spaces is accomplished by the diagram

$$
\begin{aligned}
& \begin{array}{c}
H^{0}\left(T \mathbb{P}_{1}, \mathcal{O}\left(m_{p}+m_{p-1}\right) \mathscr{I}\left(S_{p, p-1}\right)\right) \\
H^{0}\left(S_{p}, \mathcal{O}\left(m_{p}+m_{p-1}\right)\left[-S_{p, p-1}\right]\right) \\
H^{0}\left(S_{p-1}, \mathcal{O}\left(m_{p}+m_{p-1}\right)\left[-S_{p, p-1}\right]\right)
\end{array} \\
& \cup \\
& \text { II } \\
& Y_{p-1}\left(\mu_{p}\right),
\end{aligned}
$$

where the left diagonal map is an isomorphism. Lifting back and pushing down defines an isomorphism from $Y_{C}$ to $Y_{p-1}\left(\mu_{p}\right)$.

If we restrict to $C_{x}$ and use the proposition we have

$$
\begin{aligned}
& \simeq H^{0}\left(C_{x}, \mathcal{O}\left(m_{p}+m_{p-1}\right)[-\underbrace{S_{p, p-1}}]\right) \\
& Y_{B} \oplus Y_{C} \subset Y_{p}\left(\mu_{p}\right) \quad Y_{p-1}\left(\mu_{p}\right),
\end{aligned}
$$

so a bounded solution is continuous if its component in $Y_{C}$ and its component in $Y_{p-1}\left(\mu_{p}\right)$ are related by this map. The patching condition follows tautologically and so $H^{0}\left(C_{x}, \mathcal{O}\left(m_{p}+m_{p-1}\right)\left[-S_{p, p-1}\right]\right)$ really does correspond to $U_{p}$.

Consider now the case of $m_{p}-m_{p-1}=k_{p}=0$. There is no problem with decay behaviour; as for patching, the identification of $V_{p}\left(\mu_{p}\right)$ and $V_{p-1}\left(\mu_{p}\right)$ induces an identification of $Y_{p}\left(\mu_{p}\right)$ and $Y_{p-1}\left(\mu_{p}\right)$. If we start with a section in $H^{O}\left(C_{x}, \mathcal{O}\left(m_{p}+m_{p-1}\right)\right.$ $\left.\left[-S_{p, p-1}\right]\right)$ its images in $Y_{p}\left(\mu_{p}\right)$ and $Y_{p-1}\left(\mu_{p}\right)$ are related by the pulling back and pushing down described above. As $k_{p}=0, m_{p}+m_{p-1}=2 m_{p}=2 m_{p-1}$, and there is an ambiguity in lifting back a section from $C_{x} \cap S_{p}$ to $C_{x}$, namely all the multiples of $g_{p}$; similarly, that in lifting from $C_{x} \cap S_{p-1} C_{x}$ is $g_{p-1}$. The net effect of this is that a section in the kernel of Nahm's operator arising from a section of $E$ over $C_{x}$ may have a discontinuity at $\mu_{p}$ which is a multiple of $\left(g_{p}-g_{p-1}\right)$. Comparing with (2.27) we see that this means that the discontinuity is in the image of $\underline{A}^{+}(\zeta)$ $A^{-}(\zeta)$, which is precisely the result required. Again $U_{p}$ is identified with $H^{0}\left(C_{x}, \mathcal{O}\left(m_{p}+m_{p-1}\right)\left[-S_{p, p-1}\right]\right)$.

4d) The Equivalence of the Connections and of the Higgs Fields. The isomorphism of (4.6) and (4.6a) now yields an isomorphism of two bundles over $R^{3}$,

$$
C(x)=H(x)=H^{0}\left(C_{x}, E\right),
$$

each of which is equipped with a solution to the Bogomoln'yi equations. We complete the discussion by showing that the connections and Higgs fields are equivalent.

Recall from [Hu1] that fixing a direction $\left(0,0, x_{3}\right)$ in $\mathbb{R}^{3}$ means looking at a family of real sections in $T \mathbb{P}_{1}$ all intersecting on $\mathbb{P}_{1}$ in the same two points, 0 and $\infty$. We can trivialize the bundle $E$ over any of these real sections by evaluation at either of these points and define two "evaluation" connections $\nabla_{0}$ and $\nabla_{\infty}$ in $H$ along $\left(0,0, x_{3}\right)$. Then these relate to the connection and Higgs field by 


$$
\Phi d x^{3}=\frac{i}{2}\left(\nabla_{0}-\nabla_{\infty}\right), \quad \nabla_{x^{3}}=\frac{1}{2}\left(\nabla_{0}+\nabla_{\infty}\right) .
$$

Notice that if we know these two quantities for every choice of line, then the connection and Higgs field are completely determined, and vice versa. It is enough then to show that under the identification of $C$ with $H$, both constructions give rise to the same $\nabla_{0}$ and $\nabla_{\infty}$ or, because of the symmetry, the same $\nabla_{0}$.

From Nahm's point of view the operator $\nabla_{0}$ is defined by $\pi\left(\partial / \partial x_{3}+t\right)$, where $\pi$ is the orthogonal projection onto the cokernel of $\mathscr{D}$. To establish the equivalence we will show that a section constant for Nahm's $\nabla_{0}$ when interpreted on $T \mathbb{P}_{1}$ via the isomorphism (4.17) is constant for the twistor $\nabla_{0}$, that is it takes a constant value in the fibre of $E$ over 0 .

Suppose for simplicity that none of the $k_{p}$ are zero. Let $f\left(t, x_{3}\right)=f=\left(f_{1}, \ldots, f_{N-1}\right)$ lie in $\operatorname{coker}(\mathscr{D}(x))$ and suppose that $\pi\left(\partial / \partial x_{3}+t\right) f=0$. There then exists a $g=$ $\left(g_{1}, \ldots, g_{N-1}\right) \in \mathscr{H}$ with $\left(\partial / \partial x_{3}+t\right) f_{p}=D_{p}(x) g_{p}$ for all $p$. Expanding the $2 \times 2$ matrices in $D_{p}^{*}$, we find

$$
\left[D_{p}^{*}\left(x_{3}\right), \frac{\partial}{\partial x_{3}}+t\right]=\left[\begin{array}{ll}
0, & 0 \\
0, & 2
\end{array}\right]
$$

Writing $f_{p}=\left(f_{p}^{\prime}, f_{p}^{\prime \prime}\right)^{T}$, we have that $\mathscr{D}^{*} \mathscr{D} g=\left(0,2 f^{\prime \prime}\right)^{T}$. From the positivity and reality of $\mathscr{D}^{*} \mathscr{D}$ it follows that $g=\left(0, g^{\prime \prime}\right)$ and therefore

$$
\left(\frac{\partial}{\partial x^{3}}+z\right) f_{p}=-\left[\begin{array}{c}
\left(T_{1}+i T_{2}\right) g_{p}^{\prime \prime} \\
h_{p}
\end{array}\right]
$$

for some $h_{p}$.

If we think of these as sections over $T \mathbb{P}_{1}$ we can use 1 and $\zeta$ as a basis for $\mathbb{C}^{2}=H^{0}\left(T \mathbb{P}_{1}, \mathcal{O}(1)\right)$ and obtain

$$
\left(\frac{\partial}{\partial x^{3}}+t\right) f_{p}=-\left(T_{1}+i T_{2}\right) g_{p}^{\prime \prime}+\zeta h_{p}=-A_{0} g_{p}^{\prime \prime}+\zeta h_{p}=-\left(\eta-\zeta A_{1}-\zeta^{2} A_{2}\right) g_{p}^{\prime \prime}+\zeta h_{p},
$$

where $f_{p}=f_{p}^{\prime}+\zeta f_{p}^{\prime \prime}$. To obtain a section over $C_{x}$ we have to change the trivialization to $F_{p}=\exp \left(x_{3} t\right) f_{p}$, and therefore using this and Eq. (4.20) we obtain

$$
\frac{\partial F_{p}}{\partial x_{3}}=-e^{t x_{3}}\left(\eta-\zeta A_{1}-\zeta^{2} A_{2}\right) g_{p}^{\prime \prime}+e^{t x_{3} \zeta h_{p}} .
$$

Evaluating at $\zeta=\eta=0$ we see that $F_{p}$ is constant in the $x_{3}$ direction as required. The case when some of the $k_{p}$ are zero is proven similarly.

\section{Modifications for the Cases $S O(k), S p(k)$}

In this section, we briefly summarize the modifications necessary for treating the cases of $S O(k), S p(k)$.

5a) From Monopoles to Spectral Data. We now suppose that the bundle $H$ over 
$\mathbb{R}^{3}$ is equipped with a (symmetric or skew) bilinear form, compatible with the unitary structure, preserved by the connection, with respect to which the Higgs field is skew adjoint. The asymptotics of the Higgs field satisfy $\mu_{i}=-\mu_{N-1+1}$, $k_{i}=-k_{N-i+1}$. Along a line, the form applied to a pair of solutions $s, s^{\prime}$ of $(\nabla-i \Phi) s=0$ is constant; the bilinear form thus passes over to a bilinear form ( , ) defined on the bundle $E$ over $T P_{1}$. Alternately, one has an antilinear map

$$
J: E \rightarrow E
$$

lifting the map $\tau$. Composing with the map $\sigma$ of $1 e$ ), one has a holomorphic bundle map $\sigma J: E \rightarrow E^{*}$. The form is then given by $(a, b)=\sigma J(a)(b)$. In the orthogonal case, $J^{2}=1$; in the symplectic case $J^{2}=-1$.

As the flags are defined by decay rates at $\pm \infty$ of solutions to $(\nabla-i \Phi) s=0$, evaluating the bilinear form near $\pm \infty$ gives us:

$$
\left(E_{p}^{ \pm}\right)^{\perp}=\left(E_{N}^{ \pm}-p\right)
$$

i.e. the flags are "isotropic-coisotropic." As a consequence, $\left(\left(E_{p}^{+} \cap E_{N-p}^{-}\right)=0\right) \Leftrightarrow$ $\left(\left(E_{n-p}^{+} \cap E_{p}^{-}\right)=0\right)$, i.e.

$$
S_{p}=S_{N-p}
$$

Similarly,

$$
\begin{aligned}
& S_{p, p+1}=S_{N-p, N-p-1} \\
& S_{p+1, p}=S_{N-p-1, N-p} .
\end{aligned}
$$

In $[M]$, spectral curves $R_{p}$ are defined for $G$-monopoles, $G$ any compact Lie group. Some Lie theory then shows that in our case, the curves $R_{p}$ and $S_{q}$ are linked by the relations given in the introduction.

The existence of monopoles with the $R_{q}$ in general position is proven in the same way as in Sect. 1. In the case of $\operatorname{Sp}(k)(N=2 k)$, it then follows that the curves $S_{p}$ are in general position, and the whole of Sect. 1 goes through verbatim.

In the orthogonal case, one must recompute some of the quotients in (1.12). For $S O(2 k)$, the isomorphisms $C-1$ (proven in $[M]$ ), give us, over $R_{+} \cap R_{-}$, an isomorphism $L^{-\mu_{k}}\left(m_{k-1}\right)=L^{\mu_{k}}\left(m_{k-1}\right)$. Using this, one has:

$$
\begin{aligned}
& 0 \rightarrow E /\left.\left.\left(E_{k}^{+}+E_{k}^{-}\right) \rightarrow\left\{L^{\mu_{k}}\left(m_{k-1}\right) \oplus L^{-\mu_{k}}\left(m_{k-1}\right)\right\}\right|_{R_{+}} \rightarrow L^{\mu_{k}}\left(m_{k-1}\right)\right|_{R_{+} \cap R_{-}} \rightarrow 0, \\
& 0 \rightarrow E /\left.\left.\left(E_{k-1}^{+}+E_{k}^{-}\right) \rightarrow L^{\mu_{k}}\left(m_{k-1}\right) \oplus\left\{L^{-\mu_{k}}\left(m_{k-1}\right)\right\}\right|_{R_{+}} \rightarrow L^{\mu_{k}}\left(m_{k-1}\right)\right|_{R_{+} \cap R_{-}} \rightarrow 0, \\
& \left.0 \rightarrow E /\left(E_{k}^{+}+E_{k-1}^{-}\right) \rightarrow\left\{\left.L^{\mu_{k}}\left(m_{k-1}\right)\right|_{R_{+}}\right\} \oplus L^{-\mu_{k}}\left(m_{k-1}\right)\right\}\left.\rightarrow L^{\mu_{k}}\left(m_{k-1}\right)\right|_{R_{+} \cap R_{-}} \rightarrow 0 .
\end{aligned}
$$

The other quotients are as in the unitary case. For $S O(2 k+1)$, one has from $[M]$ the isomorphism

$$
s: \mathcal{O} \approx L^{\mu_{k}}\left(m_{k-1}\right)\left[-S_{k-1, k}\right]
$$

Consider now the exact sequence:

$$
\left.\left.\left.0 \rightarrow \mathcal{O}\left(-m_{k}\right)\right|_{R_{k}} \rightarrow \mathcal{O}\right|_{2 R_{k}} \rightarrow \mathcal{O}\right|_{R_{k}} \rightarrow 0
$$

and tensor it by $L^{\mu_{k}}\left(m_{k-1}\right)\left[-S_{k-1, k}\right]$. The coboundary $\delta(s)$ in

$$
H^{1}\left(R_{k}, L^{\mu_{k}}\left(m_{k-1}-m_{k}\right)\left[-S_{k-1 . k}\right]\right) \approx H^{1}\left(R_{k}, \mathcal{O}\right)
$$


defines an extension $P_{k}$ over $T \mathbb{P}_{1}$ :

$$
\left.0 \rightarrow \mathcal{O}\right|_{R_{k}} \rightarrow P_{k} \rightarrow \mathcal{O}\left(m_{k}\right) \rightarrow 0 \text {, }
$$

one has $P_{k} \approx E /\left(E_{k}^{+}+E_{k}^{-}\right)$. All the other quotients are as in (1.12).

From this point, the vanishing theorems, as well as the asymptotic estimates on the Higgs field, follow more or less as in the unitary case.

One must also show that one can construct an appropriate form on a bundle built starting from the spectral data, as in (1.14). It is easiest, in fact, to build the map $J$. Some thought shows that $J$ should descend to the sum of the quotients $P_{i}=E /\left(E_{i}^{+}+E_{N-i-1}^{-}\right)$, interchanging $P_{i}$ and $P_{N-i+1}$. In the $\operatorname{Sp}(k)$ case, for example, this amounts to finding a $J: L^{\mu_{2}}\left(m_{i-1}+m_{i+1}\right) \otimes \mathscr{I}\left(S_{i-1, i}\right) \rightarrow$ $L^{-\mu_{1}}\left(m_{i-1}+m_{i+1}\right) \otimes \mathscr{I}\left(S_{i, i-1}\right)$ lifting $\tau$, which certainly is possible.

5b) From Spectral Data to Nahm Data. Given the spectral data, the next step is to construct bundles $X$ over the intervals $\left(\mu_{i+1}, \mu_{i}\right)$, as well as a solution to Nahm's equations over these intervals. This is done in essentially the same way as in Sect. 2 , the proof that the boundary conditions are satisfied being modified slightly to take the different structure of the quotients $Q_{p}=E /\left(E_{p}^{+}+E_{N-p}^{-}\right)$into account.

One must also construct the matrices $C_{j}$ of condition B-3. Invariantly, this is equivalent to giving a pairing of $X_{z}$ with $X_{-z}$, covariant constant with respect to the connection on $X$, and such that $T_{i}(z)$ and $T_{i}(-z)$ are adjoints of one another. As above, this form is most easily defined by giving an antilinear map $\tilde{J}: X_{z} \rightarrow X_{-z}$, and then using the unitary structure. Note that for $z \in\left(\mu_{i+1}, \mu_{i}\right), X_{z} \approx$ $H^{0}\left(S_{i}, Q_{i} \otimes L^{-z}(-1)\right)$. To define $\widetilde{J}$, one uses the map $J$ given above; $J$ can be "pushed down" to a map $J^{\prime}: Q_{i} \rightarrow Q_{N-i}$; one also has a map $J^{\prime \prime}: L^{z}(-1) \rightarrow L^{-z}(-1)$, with $\left(J^{\prime \prime}\right)^{2}=-1 . \widetilde{J}$ is then the map induced on sections by $J^{\prime} \otimes J^{\prime \prime}$; one has $\left(\tilde{J}^{2}= \pm 1\right)$ $\left(J^{2}=\mp 1\right)$, and so $\widetilde{J}^{2}=1$ for $S p,-1$ for $S O$. An alternative definition of the form is given in [Hu3].

\section{Summary and Conclusion}

We have now built up all the ingredients of our theorem; it is perhaps appropriate to sum up by showing how they all fit together to give the desired result.

First, the generic case. In Sect. 1, we showed how a generic monopole gave one spectral data; we also showed that the map was injective; the inverse (twistor) construction gives back the original monopole. In Sect. 2 and 3, we proved that there is an equivalence between spectral data and generic Nahm data. In Sect. 4, we showed that any spectral data yielded back a monopole. This is done by building a bundle $E$ on $T \mathbb{P}_{1}$, and applying the twistor transform. To see that applying the construction of Sect. 1 gives back the same spectral data, one notes that the bundle one obtains from the monopole by the inverse twistor transform must be $E[\mathrm{Hi1}]$; to see that the spectral data is the same, it is sufficient to show that the flags $E_{i}^{+}, E_{i}^{-}$are the same. Referring to Sect. 1d on the asymptotic Higgs field, one sees that this is indeed the case, as the sum of the asymptotic eigenspaces corresponding to $\mu_{1} \cdots \mu_{i}$ is indeed $E_{i}^{+}$; and similarly, for $E_{i}^{-}$. Alternately, one can apply the result of [HiM], showing that the spectral data is the same. Thus, theorem 1 is proven. 
In the non-generic case, Sect. 2 showed that a monopole gives a solution to Nahm's equations; if the monopole is a limit of generic monopoles, then the solution satisfies the boundary conditions. Conversely, given Nahm data, we showed in Sect. 4 how to construct a solution to the Bogomolny equations over $\mathbb{R}^{3}$. The set of Nahm data is connected [Hu3] and so our Nahm data is the limit of generic Nahm data with spectral curves of the same degree. If one examines the construction, it is easy to see that the solution to the Bogomolny equations is a limit of monopoles. The monopole version of the Uhlenbeck compactness theorem [AHi, Proposition 3.9] implies that this limit is a monopole of possibly lower charge. However, if the charge is lower, this implies that the spectral curves do not stay bounded as we approach our limit, which is precluded in this case. Alternately, one could use an improved formula for the asymptotic Higgs field, with explicit bounds on the exponential error term; note that this formula remains defined in the limit, as it involves only the geometry of the spectral curves.

The circle therefore closes, giving one various points of view for attacking the problem. Each construction highlights certain aspects: the twistor viewpoint emphasizes the role of algebraic curves, and gives us asymptotic behaviour quite neatly; the regularity, however, is easiest to see from the Nahm viewpoint. This latter is also the most convenient for computing moduli [Hu3].

Several problems remain: one is showing that this construction yields all monopoles. This is equivalent to showing that the monopole moduli space for fixed charge is connected; it seems quite likely that this is the case [T2]. Another problem is extending these ideas to arbitrary groups, and to non-maximal symmetry breaking $\left(\mu_{i}\right.$ not distinct.)

Acknowledgements. Both authors would like to thank the Institute for Advanced Study for its hospitality during the preparation of this paper.

\section{References}

[AHH] Adams, M., Harnad, J., Hurtubise, J.: Isospectral Hamiltonian flows in finite and infinite dimensions: II. Integration of flows. Preprint

[AHi] Atiyah, M. F., Hitchin, N. J.: The geometry and dynamics of magnetic monopoles. Princeton: P.U.P. 1988

[D] Donaldson, S. K.: Nahm's equations and the classification of monopoles. Commun. Math. Phys. 96, 387-408 (1984)

[JT] Jaffe, A., Taubes, C. H:: Vortices and monopoles. PPh2, Boston: Birkhaüser 1980

[Ha] Hartshorne, R.: Algebraic geometry. GTM vol. 52. Berlin Heidelberg, New York: Springer 1977

[Hi1] Hitchin, N. J.: Monopoles and geodesics. Commun. Math. Phys. 83, 579-602 (1982)

[Hi2] Hitchin, N. J.: On the construction of monopoles. Commun. Math. Phys. 89, 145-190 (1983)

[Hi3] Hitchin, N. J.: Linear field equations on self dual spaces. Proc. R. Soc. Lond. A370, 173-191 (1980)

[HiM] Hitchin, N. J., Murray, M. K., Spectral curves and the ADHM construction. Commun. Math. Phys. 118, 463-474 (1988)

[Hu1] Hurtubise, J. C.: The asymptotic Higgs field of a monopole. Commun. Math. Phys. 97, 381-389 (1985)

[Hu2] Hurtubise, J. C: Monopoles and rational maps: A note on a theorem of Donaldson. Commun. Math. Phys. 100, 191-196 (1985)

[Hu3] Hurtubise, J. C.: The classification of monopoles for the classical groups. Commun. Math. Phys. 120, 613-641 (1989) 
[M] Murray, M. K : Non-Abelian magnetic monopoles. Commun. Math. Phys. 96, 539-565 (1984)

[N] Nahm, W.: The construction of all self-dual multi-monopoles bys the ADHM method, in Monopoles in quantum ficld theory. Craigie et al. (ed.). Singapore: World Scientıfic 1982

[T1] Taubes, C. H.: The existence of multi-monopole solutions to the non-abelian Yang-Mills Higgs equations for arbitrary simple gauge groups. Commun. Math. Phys. 80, 343 (1981)

[T2] Taubes, C. H.: Min-max theory for the Yang-Mills-Higgs equations. Commun. Math. Phys. 97, 473-540 (1985)

[W] Ward, R. S.: Ansätze for self-dual Yang-Mills fields. Commun. Math. Phys. 80, 563-574 (1981)

Communicated by A. Jaffe

Received May 27, 1988; in revised form September 1, 1988 
